\title{
Dynamiken in der Gruppenbildung der Kurden durch nationalstaatliche Prozesse
}

Eine politikethnologische Betrachtung Irakisch-Kurdistans

\author{
Hausarbeit \\ zur \\ Erlangung des Magistergrades (M.A.) \\ der sozialwissenschaftlichen Fakultät der Georg-August-Universität \\ Göttingen
}

\author{
vorgelegt von \\ Jennifer Jasberg \\ geb. in Gelsenkirchen
}

1. Hauptfach Ethnologie

Überarbeitete Fassung November 2009 


\section{$\underline{\text { Inhaltsverzeichnis }}$}

\section{EINLEITUNG}

\section{THEORETISCHE GRUNDLAGEN}

1. Konstruktivistische Ansätze zu Gruppenbildung und sozialer Identität 5

1.1 Gruppenbildung und -bindung: Ein aktueller Forschungsgegenstand 6

1.2 Soziale Identität

2. Zur Konstruktion von Ethnizität 10

2.1 Die Abhängigkeit von Zuschreibungen 11

2.2 Merkmale von Ethnien $\quad 12$

3. Von der Nation zu internationaler Staatlichkeit $\quad 15$

3.1 Die verwirklichte Nation und Nation-Building 16

3.1.1 Nationalismus: Katalysator für westliches Nation-Building $\quad 16$

$\begin{array}{ll}\text { 3.1.2 Nationalismus und Kapitalismus } & 19\end{array}$

3.2 Nationale Identität $\quad 20$

3.3 Ethnische Konflikte und nationale Grenzen $\quad 22$

3.4 Zur Politik des Nation-Building 25

3.4.1 Die Theorie des Demokratischen Friedens 26

$\begin{array}{ll}\text { 3.4.2 Strukturwandel und Institutionenbildung } & 27\end{array}$

3.4.3 Politische Eliten: Gewinner und Verlierer 28

$\begin{array}{lr}\text { II. KURDEN IM IRAK } & 30\end{array}$

1. Ethnische Marker des Kurdentums 30

1.1 Kurdistan: Der territoriale Bezug $\quad 31$

1.2 Kurdisch: Eine gemeinsame Sprache?

1.3 Kurdische Kultur $\quad 35$

1.4 Religionszugehörigkeiten 36

2. Die Genese Irakisch-Kurdistans: Ein historischer Überblick 37

2.1 Die Ausgangssituation: Kurden vor dem ersten Weltkrieg 38

2.2 Nation-Building des Iraks $\quad 38$

2.3 Konfliktsteigerung unter dem zweiten Baath-Regime $\quad 42$

2.4 Von der Flugverbotszone zur kurdischen Autonomieregion 43

III. ZU GRUPPENORGANISATIONEN UND -DYNAMIKEN 46

1. Außerstaatliche sozio-politische Strukturen 46

$\begin{array}{ll}1.1 \text { Verwandtschaftsbeziehungen } & 46\end{array}$

$\begin{array}{ll}1.2 \text { Nomadismus und tribale Strukturen } & 48\end{array}$

$\begin{array}{ll}1.3 \text { Junge und alte „Dynastien“ } & 50\end{array}$ 
2. Identitätsstiftende Momente für die Bevölkerung Iraks 52

2.1 Irakisierung, Panarabismus und die Arabisierung Iraks 53

2.2 Sozialismus und Kalter Krieg

2.3 Politisierung (islamischer) Glaubensgemeinschaften $\quad 57$

2.4 Modernisierung der Infrastruktur $\quad 59$

2.5 Internationalisierung: Emigration und Diaspora 60

3. Integration nationalstaatlicher Strukturen und Ideale 61

3.1 Nationale Strukturierung und Institutionenbildung $\quad 62$

3.2 Nationale Symbole und Rituale $\quad 63$

3.3 Politische Repräsentation durch Parteien $\quad 64$

3.3.1 Die KDP (Kurdish Democratic Party) 66

3.3.2 Die PUK (Patriotic Union of Kurdistan) 67

$\begin{array}{ll}\text { 3.3.3 Elitenrekrutierung } & 68\end{array}$

3.4 Nationalisierung durch andere politische Organisationseinheiten 69

$\begin{array}{ll}3.5 \text { Peschmerga } & 70\end{array}$

3.6 Irakisch-Kurdistan: Eine Absage an Groß-Kurdistan? $\quad 71$

$\begin{array}{lr}\text { FAZIT } & 73\end{array}$

$\begin{array}{ll}\text { Ausblick } & 76\end{array}$

$\begin{array}{ll}\text { ANHANG } & 78\end{array}$

$\begin{array}{ll}\text { I.a Die Wilayats des Osmanischen Reichs - späterer Irak } & 78\end{array}$

$\begin{array}{ll}\text { I.b Die Region Irakisch-Kurdistan } & 79\end{array}$

II.a Kurdische „Gruppen“ im Irak $\quad 80$

$\begin{array}{lr}\text { II.b.Kurdische Stämme } & 81\end{array}$

III.a Kurdische Sprachen $\quad 81$

III.b Unterteilung der kurdischen Sprache in ihre Mundarten nach Havrest (1998) 82

IV.a Irakische Bevölkerung nach Religionszugehörigkeiten $\quad 82$

IV.b Religionen der Kurden $\quad 84$

V. Kurdistan der Parteien $1991 \quad 85$

VI. Erdölregion Kurdistan $\quad 86$

VII. Beziehungen im Kalten Krieg $\quad 87$

VIII. Parteien um 1980 im Überblick $\quad 88$

$\begin{array}{ll}\text { IX. Positionen - Prestige } & 89\end{array}$

$\begin{array}{lr}\text { LITERATURVERZEICHNIS } & 90\end{array}$ 


\section{Einleitung}

„The Kurdish nation urgently appeals to the United Nations and all its member states to dispatch forthwith a commission of enquiry to the area in order to investigate the crime of genocide being committed against the Kurdish people by the Iraqi Government. “

(Statement from the Kurdish Bureau 1962: 374)

Aus diesem von Kurden im französischen Exil formulierten Hilfegesuch aus dem Jahre 1962 geht nicht nur der Umfang des Konfliktes hervor, sondern auch die bis heute wichtige Rolle verschiedener Bezugsgruppen für die Identifikationsmöglichkeiten der Kurden im Irak: Die internationale Staatengemeinschaft, das kurdische Volk, die kurdische Nation, die kurdische Diaspora und die Irakische Regierung.

Ein einzelner Mensch ordnet sich für gewöhnlich Gruppen zu, deren Strukturen auch seine eigene Handlungsfähigkeit prägen; eine solche (Bezugs-) Gruppe kann das Volk oder die Nation sein. Das international geltende Völkerrecht unterscheidet zwischen souveränen Völkern, die ein Anrecht auf einen eigenen Staat haben und Minderheiten, die Minderheitenschutz genießen sollen (Klugmann 2002: 38). Bei der Betrachtung der Kurden beginnt genau hier das Problem. Als Kollektiv wahrgenommen sind Kurden Bewohner von Kurdistan. Dieses Konstrukt vermag jedoch lediglich als Idee das kurdische „Volk“, das sich real über verschiedene Nationalstaaten verteilt, zu umfassen. Und obgleich der Begriff schon viel politische Sprengkraft und diversen Akteuren Anreiz zu Politisierungen bietet, so ist doch auch ein klar erfassbares und sich in zunehmendem Maße stabilisierendes Konstrukt mit eben dieser Betitelung zu finden: Irakisch-Kurdistan (vgl. Anhang I.b). Als autonome Region mit einem ethnischen Stempel und als Teil des irakischen Nationalstaates fasst es derzeit, sich selbstverwaltend, Fuß inmitten der westlich gedachten modernen Staatengemeinschaft, in einer der konfliktreichsten Regionen der Welt. Nicht nur von den Betroffenen wurde bereits in den 1960er Jahren das sogenannte „Kurdenproblem“ als wesentliches im Mittleren Osten erkannt. Es zeichnete sich zunehmend als neuartige Herausforderung für die internationale Politik ab. Einer Epoche kurdischer Aufstände gegen die Osmanen (1804-1918) und der Auflehnung gegen die koloniale Eingliederung der Stämme in verschiedene moderne, staatsrechtlich orientierte Nationalstaaten folgte Mitte des 20. Jahrhunderts eine Phase des dauerhaften Aufstandes, der nicht mehr nur von einzelnen Stämmen, sondern allen Kurden im Nordirak mit dem gemeinsamen Ziel der Selbstverwaltung getragen wurde (Hottinger 1965: 465). Die Region war in den folgenden Jahrzehnten Schauplatz zahlreicher Krisen und Konflikte, die sich massiv auf das Leben der Kurden und ihre gesellschaftliche Formation 
auswirkten. Als sich Erfolge für das Entstehen einer autonomen Region abzeichneten, lähmten dort bürgerkriegsähnliche Zustände weitreichende Entwicklungen.

In den letzten beiden Jahrzehnten haben Kurden im Irak allerdings einen bis dahin unereichten Grad an politischer Eigenständigkeit erlangt, der Regierung, Verwaltung und Gerichtsbarkeit umfasst, wobei sie als Allianz gegen die irakische Zentralregierung 2003 an der Seite der Amerikaner gegen das Irakische Regime in den Krieg zogen. Ungeachtet dessen, dass sich der Irak momentan als demokratischer, föderaler Staat zu etablieren scheint, sagt das noch nichts über die regionale Verfasstheit, politische Struktur oder Norm und Zugehörigkeitsempfinden der Betroffenen aus. Schwierigkeiten in dieser Entwicklung werden unter anderem damit beschrieben, dass nationale Kohäsion durch tribale oder religiöse Einflüsse dominiert würde (Bengio 2005: 179).

Die vorliegende Arbeit hat zum Ziel, die Prozesse, die auf die kurdischen Gruppen und ihre Zusammensetzungen in der Region Irakisch-Kurdistan gewirkt haben, zu erfassen, nachzuzeichnen und gestützt auf sozialwissenschaftliche Theorien zu bewerten. Es wird der Frage nachgegangen, welche Aussagen über Gemeinschaftsorganisation und -repräsentation im Aufeinandertreffen von moderner Nationalstaatlichkeit und praktisch gelebter Gruppenorganisation am Beispiel der Kurden im Irak getroffen werden können.

Eine anfängliche Begutachtung des vergangenen Jahrhunderts und seiner Akteure lenkt schnell die Aufmerksamkeit auf die häufige Wiederkehr bestimmter Familiennamen, die unabhängig vom politischen Kontext auffällt, und wirft die Frage nach Identifikationsmöglichkeiten der „Bürger“ auf. Einfache Erklärungen, die das Aufeinanderprallen europäisch konzipierter Staatskonzepte auf „traditionelle“ kollektive Bindungsmuster als einen Konflikt des „Entweder...oder“ beschreiben, können nicht die Stabilität bestimmter kulturspezifischer Gruppenformen und Hierarchien inmitten des sich zusehendst konsolidierenden modernen Teilstaates erklären.

Eine Beschränkung bei der ethnologischen Betrachtung auf die irakischen Kurden erfolgt aufgrund des nur bei ihnen möglichen langfristigen Abgleichs mit dem sich herausbildenden Staatskonzept. Zudem kann diese Arbeit, da sie theoretisch angelegt ist, lediglich verallgemeinernd Strukturen und wenige Daten der Mikro-Ebene präsentieren. Dabei verstehe ich die Arbeit eher als einen Überblick und weniger als eine genaue Analyse von Integrationsprozessen beispielsweise der Kurden insgesamt. Eine politikethnologische Betrachtung dieser Entwicklungen sollte jedoch als Grundstein für die im Kleineren anzufertigenden konkreteren Feldstudien gesehen werden können. 
Festgestellt wird, dass die Arbeit nicht gewährleisten kann, die vielen verschiedenen, teils sehr subjektiv gefärbten, Positionen und zeitabhängigen Forschungsstände umfassend zu berücksichtigen, da es vordergründig darum geht, Daten $\mathrm{zu}$ sammeln, die einen Gesamteindruck der sozialen Gegebenheiten vermitteln können.

Einleitend werden in einem ersten Teil die theoretischen Grundlagen einer konstruktivistischen Betrachtung der Genese von Ethnizität, modernen Nationalstaaten und nationaler Identität geliefert. Im mittleren Teil der Arbeit werden grundlegende Informationen über den historischen Kontext sowie, entsprechend der Theorie, der ethnische Charakter der Kurden vorgestellt, um dann konkret die soziale und politische Organisation der irakischen Kurden sowie identitätsprägende Entwicklungen und die Integration in moderne nationalstaatliche Modelle zu diskutieren.

Was die Quellenlage betrifft, so ist diese für mein Anliegen überschaubar. ${ }^{1}$ Ethnologisch angelegte Arbeiten über Kurden im Irak liegen aus den 60er Jahren von Barth und aus den 80ern von Franz vor. Allgemein nahm das Interesse an den Kurden und ihrer Identität nach Kriegsbeginn im Irak 2003 zu. Auf neuere, ausführliche wissenschaftliche Abhandlungen kann dank Maria O’Shea (2003), Salih (2005) und Asadi (2007) zurückgegriffen werden. ${ }^{2}$

Wesentlich lässt sich feststellen, dass die ethnologische Literatur nur in geringem Maße ökonomische oder politische Kontexte erfasst. ${ }^{3}$ Da jedoch die von Kurden bewohnte Region vor allem seit dem Ersten Weltkrieg in Bezug auf militärische und wirtschaftliche Strategien das Interesse der Europäer geweckt hatte, erschienen zahlreiche sozialwissenschaftliche Publikationen in eben diesem Sinne.

Zur Veranschaulichung der Inhalte vor allem mit regionalem Bezug befinden sich Karten im Anhang. Hierzu sei angemerkt, dass diese gewiss Machtinteressen widerspiegeln.

Bei Namen, die aus dem Kurdischen, Persischen und Arabischen entlehnt sind, wird, insofern diese Arbeit nicht sprachwissenschaftlich angelegt ist, auf die Transliteration verzichtet. Ich nutze bei den Begriffen, wenn möglich, die für den deutschen Leser verständlichste oder die in der Literatur am häufigsten gebrauchte Schreibweise. Weicht diese von der Transliteration gemäß der Deutschen Morgenländischen Gesellschaft sehr ab oder steht der Begriff im Zentrum der Erläuterung, füge ich sie in Klammern an.

\footnotetext{
${ }^{1}$ Aufgrund schlechter Zugänglichkeit und mangelnder Katalogisierung und Verschlagwortung der Thematik in universitären Bibliotheken stammen meine Quellen überwiegend aus der Recherche in der Bibliothek des Deutschen Bundestages.

${ }^{2}$ Verwiesen sei an dieser Stelle auf die bibliographische Übersicht zu kurdischer Kultur von Meho und Maglaughlin (2001 Kurdish Culture and Society. London: Greenwood Press), die umfangreich auch neue Aufätze und Titel auflistet, jedoch darunter viele, die in Deutschland nicht ohne erheblichen Aufwand erhältlich sind. Der umfassendste Überblick über kurdische Organisationen und Persönlichkeiten stammt von Franz, 1987.

${ }^{3}$ Einen weiten Überblick über den Forschungsstand bietet Bahjat 2001 in der Einleitung.
} 


\section{Theoretische Grundlagen}

Der ethnologische Zugang zur Fragestellung soll vor allem durch den theoretischen Unterbau der Analyse gewährleistet werden. Insofern die Auseinandersetzung mit Entwicklungen, die durch dynamische Prozesse der Nationalstaatsgründungen im Mittleren Osten geprägt sind, im Vordergrund der Arbeit steht, begreife ich sie als politikethnologisch. Entsprechend der Definition von Heidemann beschäftigt sich Politikethnologie ,, mit Politik in fremden Kulturen sowie zwischen Kulturen und führt einen kulturvergleichenden Diskurs über Aspekte des Politischen, über Herrschaft, Macht, Hierarchie etc.“ (2003: 157). Er weist damit auf das Dilemma hin, dass Politik nicht ausschließlich als jenes Handeln, das sich in westlichen Nationalstaaten erkennen lässt, zu verstehen sei. Erschwerend kommt hinzu, dass in vielen Gesellschaften die politische Sphäre als gesellschaftliches Teilgebiet keine entsprechend ähnlich klaren Konturen besitzt und in religiöse oder verwandtschaftliche Beziehungen und Vorstellungen eingebunden ist. Die separate Betrachtung von Politik als Handeln zum Zwecke der Machtausübung bedarf daher einer holistischen Perspektive, um komplexe Zusammenhänge, die politische Legitimierung erst verständlich machen, erkennen zu können. Ethnologisch lässt sich dementsprechend Politik definieren als ,jegliches Handeln, das die Herstellung, Veränderung oder bewusste Wahrung von allgemeinen Verbindlichkeiten, Normen, Regeln und Gesetzen beabsichtigt oder bewirkt. “ (Heidemann 2003: 160).

Im Rahmen dieser Magisterarbeit, die ohne eigene Feldforschungsdaten auskommen muss, kann zwar keine Aussage über die Intentionen politischer Akteure gemacht werden und somit auch die Rolle von Macht und Handlungsmöglichkeiten politischer Akteure nur randläufig behandelt werden, es sollen jedoch Institutionen, Systeme und Repräsentationsformen erfasst und deren Genese im Kontext der Weltanschauungen und identitätsstiftenden Kollektividentitäten nachgezeichnet werden. Da es mir um die Erfassung des Einflusses verschiedener Entwicklungen auf die Kurden als ethnische Gruppe im Zuge des Entstehens moderner Nationalstaatlichkeit geht, lege ich den Fokus auf Gruppenbildung im Allgemeinen, auf Ethnizität und Nationalität im Gröberen. Das Kapitel über Ethnizität soll den Zugang offen legen, mit dem ich versuche, die Kurden als ethnische Gruppe zu erfassen. Der Schwerpunkt der Arbeit, in dem ich mich mit den Stadien des Entstehens moderner Staatlichkeit befasse, wird sich entsprechend auf meine Ausführungen im dritten Kapitel stützen.

Der Zugriff auf konstruktivistische Theorien basiert auf der Unzulänglichkeit vorausgehender Theorieströmungen für die Behandlung dieser Thematik. Obwohl sich Neo-Evolutionismus, dessen Anhänger sich vielfach mit diesen Themen befassten, in Bezug auf das 
Politikverständnis von der Vorstellung der unilinearen Entwicklung von verwandtschaftlichen zu systemisch staatlichen Strukturen hin, verabschiedete, scheint die These eines evolutionär bedingt, höher stehenden westlichen Nationalismus populär nicht ausgestorben. Rückenwind erhält die These, da die Welt sich tatsächlich in Nationalstaaten aufteilt, ungeachtet der Funktionsfähigkeit dieser Staaten. Obschon Globalisierung die Annäherung politischer Organisationsformen forciert, werden Globalisierungsprozesse mittlerweile gemeinhin in lokale Kontexte eingebettet gesehen, wobei beide wechselseitig aufeinander wirken. Die Vereinheitlichung von Kultur, also auch politischen Ordnungsvorstellungen, weltweit als Folge vor allem ökonomischer Prozesse scheint vielen SozialwissenschaftlerInnen somit als Fehleinschätzung (Hauser-Schäublin/Braukämper 2002: 10). Ich lasse den NeoEvolutionismus auch deswegen außen vor, da er keine Antworten auf separatistische Bewegungen oder staatlichen Zerfall finden kann.

Die gewählte konstruktivistische Betrachtung geht zurück auf das Wirken postmoderner Kritiker der 1970er Jahre in der Ethnologie, was zum Überdenken der Übertragbarkeit sozialer Realität auf Gesellschaften anderer Kulturen führte. Postmoderne TheoretikerInnen haben das Politikverständnis vor allem durch ihre Sicht auf den Machtbegriff geprägt. Foucault, der davon ausging, dass Machtbeziehungen der Selbstorganisation von Gesellschaften dienten, wurde durch Niklas Luhmanns und Bourdieus These, dass Macht jedem Kommunikationsprozess inhärent sein und durch Symbole zum Ausdruck gebracht werden müsse, bekräftigt (Heidemann 2003: 172). Selbstreflektierend zeigten EthnologInnen die Unfähigkeit zur Neutralität beim Beschreiben politischer Prozesse auf.

\section{Konstruktivistische Ansätze zu Gruppenbildung und sozialer Identität}

Bevor im Folgenden Ethnie und Nation, wobei sich beides als Gruppe bezeichnen ließe, theoretisch behandelt werden, möchte ich zunächst meinen konstruktivistischen Zugang erläutern, indem ich grundlegende Überlegungen zu der Relation von Gruppe und Person voranstelle.

Bei der Beschreibung kultureller Phänomene stützen EthnologInnen sich auf essentialistische oder konstruktivistische Betrachtungsweisen. Während essentialistische, beziehungsweise primordiale Ansätze von einer objektiv fassbaren Wirklichkeit, beispielsweise einer von Individuen nicht beeinflussbaren „natürlichen“ ethnischen Identität, ausgehen, betonen konstruktivistische Ansätze die Subjektivität von Forschern und Erforschten und die kontextuelle Abhängigkeit jener Phänomene (Haller 2005: 37). Prägend für die Ende der 1960er Jahre aufkommende Betrachtung von Gruppen als soziale Konstrukte war die 
Wirkung postmoderner Sozialtheorien insbesondere aber der kritische Ansatz von Frederik Barth. Die Stärke des Arguments konstruktivistischer Positionen liegt darin, dass sie Differenzen und Veränderungen erklären können, begreifen sie doch soziale Beziehungen als konstruiert und wandelbar. So können Erklärungen für Variabilität beispielsweise ethnischer Herkunftsmythen oder neue Deutungsmuster bestimmter Gruppenzugehörigkeiten im Zuge nationalstaatlicher Entwicklungen gewagt werden (Heckmann 1997: 49f). Entsprechend der dem Titel dieser Arbeit inhärenten Frage nach den Auswirkungen politischer Veränderungen auf Gruppenbildung gehe ich auf Vergemeinschaftung und Identifizierung mit Gruppen entsprechend konstruktivistischer Theorie ein. ${ }^{4}$

\subsection{Gruppenbildung und -bindung: Ein aktueller Forschungsgegenstand}

Allgemein fordern theoretische Einsichten des sogenannten cultural turn die Methoden aller Sozial- und Kulturwissenschaften heraus. Diese verlangen die Erforschung eines Gegendstandes immer in Reflexion zur symbolischen Praxis und Sprache einer Kultur.

Nicht zuletzt Prozesse der Globalisierung lösen frühere Universaliensuche durch die nach Differenzen ab. Definitionen von Gruppen und Identitäten sind nicht mehr universell anzufertigen, was in den vergangenen Jahren besonders an der Dekonstruktion der zuvor als universell gehandelten Größe geschlechtlicher Identität deutlich wurde. ${ }^{5}$

Wenn in der heutigen Sozialwissenschaft die Rede von einer Gruppe ist, so meint das ein Gebilde, bei dem Wir-Gefühl der Mitglieder, gemeinsame Wertsetzung, Institutionen und Organe bindend wirken. Es ist also notwendig, dass das Individuum über bestimmtes Wissen verfügt und sich seiner Gruppenzughörigkeit bewusst ist, was beispielsweise Klassen von Gruppen unterscheidet (Müller 2005: 157).

Die Frage nach der Relation von Individuum und Gesellschaft, beziehungsweise Gruppe, ist ein Grundpfeiler anthropologischer Forschung. Die ebenfalls in anderen Disziplinen wie der Biologie geführten Diskussionen über die Bedeutung des Menschen als Einzel- und Gemeinschaftswesen kamen $\mathrm{zu}$ verschiedensten Ergebnissen und sind keineswegs abgeschlossen. Trotz offensichtlich zunehmender Individualisierung in der modernen Gesellschaft ist vor dem Hintergrund offener philosophischer Fragen hier nicht zu beantworten, in welchem Maße die einzelne Person wie stark an die Gesellschaft angebunden ist und inwieweit die Gemeinschaft seine persönliche Identität stiftet.

\footnotetext{
${ }^{4}$ Es bleibt zu bemerken, dass im Rahmen dieser Arbeit nur einige wenige Aspekte, die zielführend für die Bewertung der Situation irakischer Kurden sind, aus der sozialwissenschaftlichen Diskussion um den Begriff der Gruppe dargestellt werden können.

${ }^{5}$ Ohne hier die anhaltende Diskussion um Kulturrelativismus, der einen Eindruck der Beliebigkeit erzeugt, nachzeichnen zu wollen, soll berücksichtigt werden, dass auch dieser Umgang mit Kultur nicht kritiklos verhallt.
} 
In der Theorienbildung wirkte lange der Soziologe Durkheim mit seiner Annahme, dass Individualismus als Handlungsfähigkeit des Einzelnen Produkt gesellschaftlicher Arbeitsteilung sei und somit in vormodernen Gesellschaften die Unterordnung unter das Kollektiv stattgefunden habe, was bisweilen widerlegt werden konnte.

Ich möchte mich diesbezüglich auf die Ausführungen von Hechter stützen, der zwar Familie als universelle Gruppe in menschlichen Gesellschaften erfasst. Darüber hinaus jedoch gingen Menschen profitorientiert, aber nicht zwangsläufig, Beziehungen ein, denn ,the principal benefit of group formation lies in the concentration of individually held resources. By pooling at least some of their own resources, people can provide themselves with jointly produced goods - including security from predation or confiscation, cooperative production of food, insurance from natural disasters, and greater access to information and mates - that they cannot produce through their own individual efforts. Pooling also encourages specialization, which ambles such goods to be produced more efficiently. (...)The principal cost of forming a group is the subsequent loss of individual autonomy.” (2000: 20).

Über die Realität und Bindungskraft von Gruppen meint Jenkins, anknüpfend an Barth:

„To invoke the first principle of social constructionism, groups are real if people think they are: they then behave in ways that assume that groups are real and, in doing so, construct that reality." (2008: 12).

"Collective identification evokes powerful imagery of people who are in some respect(s) apparently similar to each other. People must have something in common - no matter how vague, apparently unimportant or apparently illusory - before we can talk about their membership of a collectivity. However, this similarity cannot be recognised without simultaneously evoking differentiation. Logically, inclusion entails exclusion. To define the criteria for membership of any set of objects is, at the same time, also to create a boundary, everything beyond which does not belong." (2008: 102).

Darüber hinaus weist er Organisationen, wie sie hier noch in Zusammenhang mit Ethnizität und Nationalstaat eine wichtige Rolle zu spielen haben, als reale Gruppen aus,

,which can be formal or informal, extending in size and complexity from a regular pub quiz team to a multi-national cooperation or a nation-state- are perhaps the most substantial kind of group (...) Organisations are constituted in implicit behavioural norms and customs, in explicit rules and procedure, in criteria for recruitment, in divisions of labour, in hierarchies of control and authority, and in shared objectives (...) Organisations may persist despite membership turnover. People come and go, but the organisation can continue. There is more to an organisation than its membership, and the same is true for any group or collectivity." (Jenkins 2008: 10).

Festzuhalten bleibt, dass soziale Zugehörigkeit für das Handeln eines Menschen von zentraler Bedeutung ist, denn sie stellt den Sinnbezug her und verleiht jeder Tätigkeit erst im Rahmen der Lebenswelt und der eigenen Positionierung zu Anderen einen Sinn. So kann bestimmtem Handeln in verschiedenen kulturellen Kontexten sehr verschiedene Bedeutung zukommen. Darüber hinaus finden in den Definitionen postmoderne Ansätze zu Macht und Diskursen 
ihren Niederschlag. Es gilt der Frage nachzugehen, wie und zu welchem Zweck Gruppen von Politik imaginiert werden. ${ }^{6}$

Ein Blick auf die Vielfalt aber auch Kontinuität von Namen und Namensgebungen von Familien bis hin zu Staaten verdeutlicht dabei sowohl die Pluralität von Gruppenzugehörigkeiten, wie auch dass diese nicht völlig beliebig und austauschbar sind. Lebenswelten dürfen demgemäss nicht als private Erfahrungen sondern vielmehr als etwas intersubjektiv Erfahrbares gedacht werden, das über eine Grundstruktur und Symbole verfügt.

\subsection{Soziale Identität}

„Mittlerweile gehört es zum wissenschaftlichen Gemeingut, dass der Mensch multiple, darunter inkludierende, einander umfassende Identitäten hat, welche erst die menschliche Existenz in ihrer Fülle ausmachen. " (Reiterer 1998: 14).

Der in den Sozialwissenschaften verwandte Begriff der Identität lässt sich allgemein unterteilen in eine persönliche, die eine Person aufgrund selbst und fremderlebter Kontinuität und biographischer Merkmale von Anderen unterscheidet. Dem fügt sich die soziale Identität an, die eine Person als Teil einer Gruppe beschreibt, in der sie eine soziale Position einnimmt. Die jeweilige Erfahrung des Ichs seiner Identität kann dabei sehr unterschiedlich sein und ist Gegenstand vieler ethnologischer Untersuchungen. Vorstellungen über die Verbindung von Körper(n) und Seele(n) können ebenso wie die von Tod und Leben stark variieren und bei dem Aufschluss über die Konzeption vom jeweiligen Verhältnis des Individuums zur Gesellschaft helfen (Haller 2005: 93). Das muss das Augenmerk stets auf Religion richten.

Zur Erfassung der Konstruktion sozialer Identität können auch Autoren, die im Vorfeld der theoretischen Wende Aspekte der sozialen Position betrachtet haben, herangezogen werden. So stellte bereits Goodenough 1965 fest, dass für die Verwertung ethnologisch gesammelter Daten auch Rolle und Status der Personen Berücksichtigung finden mögen.

Er definierte soziale Identität in Abgrenzung zur persönlichen als ,, an aspect of self that makes a difference in how one's rights and duties distribute to specific others. Any aspect of self whose alteration entails no change in how people's rights and duties are mutually distributed, although it affects their emotional orientations to one another and the way they choose to exercise their privileges, has to do with personal identity but not with social identity (...) every individual has a number of social identities (...) If a status is a collection of rights and duties, then the social identity we label 'physician' occupies a different status in each of these identity relationships (...) Obviously, communities, tribes and nations become parties to status relationships when they make treaties with one another and when they enter into contracts with individuals and subgroups within their membership." (1965: 3f.).

\footnotetext{
${ }^{6}$ Auch der Begriff des agency spielt immer wieder eine Rolle und thematisiert zumeist philosophisch die Handlungsfähigkeit eines Agens in der Welt. Diese sowie diskurstheoretische Überlegungen werden infolge des Mangels an Daten für eine entsprechende Analyse in dieser Arbeit nicht vorgestellt.
} 
Ungeachtet dessen, dass es in den folgenden Jahren der philosophischen und theoretischen Ausgestaltung postmodernen Gedankenguts bedurfte, um den Aspekt der Macht in den sozialwissenschaftlichen Diskurs einzubauen, zeigen damalige ethnologische Überlegungen Zusammenhänge auf zwischen der sozialen Rolle, dem Status und den möglichen Aushandlungen, die letztlich auf das Individuum als Teil einer Gruppe wirken.

Sei die Wahl sozialer Identität also konstituierend für soziale Beziehungen und gestaltete die Zuweisung eines Status die soziale Ordnung, dann stattet die Rolle den Einzelnen mit Pflichten und Rechten in der Gesellschaft aus. Die Ethnologie hat unter anderem als Aufgabe diese Ausgestaltung zu erfassen. Die Erweiterung um den Begriff des Habitus, wie ihn Bourdieu beschrieb, umfasst die Kategorisierung von Angehörigen bestimmter sozialer Klassen und deren soziale Praxis und Verhaltensstrategien. Habitus meint das notwendige Erlernen kultureller Praktiken zum Ausleben einer sozialen Identität.

Für die Thematisierung von Identitätsstiftung ist ein Blick auf die Auswirkungen des wissenschaftlichen Konstruktivismus auch in anderen Disziplinen aufschlussreich. Wesentliches Ergebnis dieser Ansätze ist, dass Wissen heute grundsätzlich im Verdacht steht perspektivisch und interessensgebunden zu sein, sowohl im Besitz des Individuums als auch der Wissenschaft. Die Verflechtung von Wissen und gesellschaftlichen Konstrukten wird besonders deutlich in der Psychologie. Galt es beispielsweise noch vor nicht allzu langer Zeit als legitim das Phänomen multipler Persönlichkeiten als krankhaft zu bezeichnen, wird nunmehr die Diagnose als Ursache für die Bewertung als Störungsmusters gesehen.

„Zur Herausforderung für die Psychologie wird all dies, wenn man die Auffassung, dass psychische "Phänomene" sowie psychologische „Diagnosen" und wissenschaftliche "Befunde" kultur- und gesellschaftsgebunden sind, methodologisch und theoretisch konsequent weiterdenkt: Dann verändert sich nämlich ihre Gegenstandsauffassung dahin gehend, dass nicht in erster Linie die „Phänomene“ (Emotionen, Kognitionen usw.) an sich $z u$ erforschen sind, sondern deren soziale Konstruktion und Transformation im alltäglichen Handeln. (...) In Diskursen und symbolischen Alltagspraktiken wird nicht etwa nur eine Art Konsens über die soziale und kulturelle Bewertung schon vorliegender psychischer Phänomene ausgehandelt. Vielmehr entsteht die Identität jener Phänomene, Prozesse, Funktionen und Zustände, deren Genese die Psychologie irrtümlicherweise ,in" das Individuum verlegt, zu einem erheblichen Teil erst in den Diskursen, in die das kollektive Wissen einer Sprach- und Kulturgemeinschaft eingeht. (Zielke 2007: 15f.).

Letzteres macht deutlich, wie sehr Diskurse, stark von wissenschaftlichen Disziplinen getragen, soziale Wirklichkeit und somit auch die Lebenswelt von Individuen gestalten. Die Teilnahme an solchen Diskursen bedarf jedoch der Gruppenzugehörigkeit, wobei die Wirkkraft auf den Diskurs von der zugeschriebenen gesellschaftlichen Rolle abhängt. ${ }^{7}$

\footnotetext{
${ }^{7}$ Wobei schon die Idee des Diskurses als .postmodernes Theoriengut mehrfach besetzt ist und im Wesentlichen die Konstituierung der Folgen von Äußerungen umfasst. Die sozialwissenschaftliche Diskursanalyse befasst sich mit authentischen Sprechereignisse im weitesten Sinne in ihrem kulturellen Kontext.
} 
Für die aktuelle sozialwissenschaftliche Auseinandersetzung mit (sozialer) Identität lässt sich auf Jenkins zurückgreifen:

"Identity is the human capacity - rooted in language - to know 'who's who`. This involves knowing who we are, knowing who others are and so on: a multi-dimensional classification or mapping of the human world and for places in it, as individuals and as members of collectivities." (2008: 5)

Jenkins geht davon aus, dass Klassifizierungen stets interessensgeleitet sind und diese Interessen seien nicht immer vorhersehbar. Kategorisierungen sozialer Art bedeuten auch über den Zugang zu Ressourcen und das Verteilen von Strafen zu bestimmen. Sie sind zentral für Konfliktbewältigung und schaffen Konfliktpotential (2008: 12).

Für die hier vorzubereitende Thematisierung von Gruppenbildung bei struktureller Veränderung halte ich abschließend fest:

"Soziale Identitäten können nicht nach Belieben erfunden werden, weil sie für andere plausibel sein müssen. Der Umfang, innerhalb dessen Identitäten verändert oder manipuliert werden können, wird durch die Systemlogik dieser semantischen Bereiche (Sprachbezeichnungen oder Namensgebung) und durch soziale Konventionen begrenzt." (Schlee 2006: 36).

Schließlich kommt keine Individualität ohne kollektive Anbindung aus, denn schon das intime Selbst identifiziert sich mit Kategorien wie Gender, Ethnizität und Verwandtschaft.

\section{Zur Konstruktion von Ethnizität}

Als eine wichtige Form sozialer Organisation und Ausdruck sozialer Identität wird nun die ethnische Gruppe und die ihr zugrunde liegende Konstruktion von Ethnizität thematisiert.

Ergebnis vieler Versuche die Gruppen, in denen Menschen sozial agieren, klassifizieren zu können, war die Genese physisch determinierbarer Begriffe wie Rasse oder Volk. Diese bezogen sich im Wesentlichen auf gemeinsame Kultur und Abstammung und dienten häufig der Exklusion bestimmter Bevölkerungsteile (Haller 2005: 95). Das Benennen einer Gruppe, die über kulturelle und historische Gemeinsamkeiten verbunden ist, als Ethnie tauchte im 19. Jahrhundert im deutschen Sprachgebrauch der Völkerkundler auf und bezog sich daher vor allem auf „fremde“ Völker. Ohne detailliert auf die Begriffsgeschichte eingehen zu wollen, möchte ich festhalten, dass Ethnie oder Ethnos in der wissenschaftlichen Auseinandersetzung in Abgrenzung zum politisierten Rassebegriff, der während des europäischen Imperialismus und Nationalsozialismus auch in akademischen Kreisen verwendet wurde, insbesondere seit der zweiten Hälfte des 20. Jahrhunderts in den Vordergrund rückte. ${ }^{8}$

\footnotetext{
${ }^{8}$ Die wissenschaftliche Beschäftigung mit Ethnizität als soziologisch erfassbarem Phänomen erlebt seit den 1960er Jahren einen Aufschwung. Jedoch wurden Soziologen wie Emerich Francis noch lange verdächtigt an zu recht problematisches rassistisches Gedankengut anzuknüpfen, während heutzutage die Diskussionen um Volk und Ethnizität infolge der Durchsetzung konstruktivistischer Ansätze üblich sind (vgl. Heckmann 1997: 46-56).
} 
Als Geertz, als wichtiger zeitgenössischer Vertreter selbstkritischer Ethnologie, Ethnizität als primordial stigmatisierte, ging es ihm um die Darstellung, dass eben die ethnische jene soziale Identität sei, die, da sie durch die Geburt frühkindlich erworben würde, die erste Zuweisung zu einem Kollektiv sei. Nicht diesem Gedanken folgend, sondern vielmehr überholte Rassekonzepte aufgreifend, findet sich noch immer vielfach in politischen Zusammenhängen die Auffassung wieder, ethnische Zugehörigkeit sei primordial und böte daher eine „natürliche“ Art der Klassifizierung (Reiterer 1998: 65). In der Folge ist fehlende Akzeptanz oder mangelnde Sichtbarkeit einer ethnischen Zugehörigkeit häufig ein Politikum.

An den konstruktivistischen Ansatz anknüpfend ist ethnische Zugehörigkeit als kollektive Identität derjenigen, die einen gemeinsamen Lebenshorizont haben, einem steten Wandel unterworfen. Als soziales Phänomen bewahrt sie tragende gesellschaftliche Grundstrukturen, auch wenn dies sehr unterschiedlich in urbanen Großgesellschaften und segmentären Kleingesellschaften beschaffen sein kann. Infolge vieler parallel verlaufender Modernisierungs- und Globalisierungsprozesse verliert Ethnizität offenbar ihren umfassenden Charakter und konkurriert mit anderen sozialen Identitäten, beispielsweise der nationalen.

Ebenso wie andere Kernbegriffe sozialwissenschaftlicher Disziplinen erlebte auch der Ethnizitätsbegriff im Zuge theoretischer Neuerungen weitgehende Transformation. Obwohl eine allgemeine Definition von Ethnizität, die auf verschiedene historische und regionale Bedingungen anwendbar wäre, schwer und wenig sinnvoll erscheint, können doch einige Aspekte zusammengetragen werden, die eher einem Fragenkatalog für bessere Orientierung gleichen. Dazu dass Ethnien sich nicht mehr über eine Liste von Merkmalen auszuweisen haben, hat wesentlich der bereits erwähnte Ansatz von Frederik Barth beigesteuert. Er fügte den zuvor gängigen Kriterien die Dimension der Zuschreibung hinzu.

\subsection{Die Abhängigkeit von Zuschreibungen}

Barths Auffassung von ethnischer Identität und Grenzen als aus- und verhandelbar und situationsabhängig gilt nun als allgemeine Grundannahme in anthropologischer Forschung (Jenkins 2008: 124). Barth zeigte auf, dass ethnische Identität der intersubjektiven Anerkennung und Fremdzuschreibung bedarf. Gruppenmitglieder müssen sich ebenso wie die Mitglieder anderer Gruppen diese Identität zuschreiben.

„It is important to recognize that although ethnic categories take cultural differences into account, we can assume no simple one-to-one relationship between ethnic units and cultural similarities and differences. The features that are taken into account are not the sum of objective` differences, but only those which the actors themselves regard as significant. Not only do ecologic variations mark and exaggerate differences; some cultural features are used by the actors as signals or emblems of differences, others are ignored, and in some relationships radical differences are played down and denied.” (Barth 1998: 14). 
Barth selbst wies in Bezug auf die Verwendung des Begriffs der Grenze (boundary) später darauf hin, dass jener sehr wohl beim analytischen Denken über Kollektive helfen möge, aber dass eine Unterscheidung zwischen Dingen nicht zwangsläufig eine definitive Grenzziehung umfasse und keineswegs sicher sei, dass alle lokalen Gruppen Kollektivität als klar abgegrenzt empfänden (Jenkins 2008: 127).

Auch Barth verstand ethnische Identität eingebunden in ein Konzept pluraler Identitäten und hielt bezüglich polyethnischer Systeme fest:

„Ethnic identity implies a series of constraints on the kinds of role an individual is allowed to play. (...) Regarded as status, ethnic identity is superordinate to most other statuses, and defines the permissible constellations of statuses, or social personalities, which an individual with that identity may assume." (Barth 1998: 17).

Ein wesentlicher Aspekt bei Barth ist die Betonung, dass kollektive Identität immer auch inhärent politisch sein müsse. So wird die Selbstzuschreibung zu einer kollektiven Identität nun aber keineswegs mehr als freiwilliger Akt des Individuums gewertet, sondern in ablehnender oder zustimmender Reaktion auf Fremdzuschreibungen (Elwert 2005: 100).

Als eine Folge des Abgrenzungsaspektes wird Ethnozentrismus als naive Haltung im Glauben an die eigene Höherwertigkeit der eigenen Ethnie gesehen. Obschon strukturalistische Vergleiche des kindlichen Egozentrismus mit Ethnozentrismus wegen der Ausblendung grundverschiedener Entwicklungsprozesse hinken, bleibt die auf das Individuum bezogene Unmöglichkeit des Denkens vom „Anderen“ doch ein Merkmal der Zentriertheit und daher besonders anfällig für politisch ambitionierte Akteure (Reiterer 1998: 53f.).

Dazu sei zu bemerken, dass sich der Vorwurf des Missbrauchs von ethnischen Zuschreibungen nicht nur an Politiker, sondern auch EthnologInnen richtet, die im Verdacht stehen, vermeintlichen Homogenisierungstendenzen entgegenzuwirken (Giordano 1997: 62).

Derzeit definieren EthnologInnen Ethnizität als einen Prozess der kulturellen Differenzierung von Bevölkerungsgruppen in Form der Selbst- und Fremdzuschreibung, wobei insbesondere Abstammung, Sprache oder Religion dem identitätsstiftenden Traditionsbewusstsein dienen können. Ethnizität wird als universelles Phänomen gewertet, das durch Inklusion und Exklusion der Sicherung von sozialer Mitgliedschaft und den Zugang zu den materiellen und ideellen Gruppenressourcen in kulturpluralistischen Staaten dient (Orywal 2005: 101).

\subsection{Merkmale von Ethnien}

Obgleich die Kriterien zur Erfassung ethnischer Identität nicht klar abgegrenzt und als konstitutiv betrachtet werden sollen, ist die Analyse von Sprache, Abstammung, Geschichte 
und Raum bei der Betrachtung einer Ethnie für das Erkennen von Transformationen oder des Nebeneinander mit anderen sinngebenden Identitäten sinnvoll.

Zum Erfassen von Ethnien hat sich das Erstellen von Merkmallisten als unzureichend erwiesen, da keineswegs jede Ethnie über die ganze Fülle der diversen gemeinsamen Merkmale verfügt, noch alle ihre Mitglieder jene in ähnlichem Maße aufweisen können. Zudem ist nicht nur in der Praxis die Bedeutung, die Individuen der ethnischen Identität beimessen, situationsgebunden, sondern auch die Hervorhebung bestimmter Merkmale von dem Bemühen sich zu Anderen abzugrenzen abhängig. Bausteine, die als ethnische Marker gerade für die Differenzierung genutzt werden, können gemeinsame Sprache, Namen, Glaube an gemeinsame Abstammung und historische Erfahrung sein. Aber auch Kleidung oder Speisen, die territoriale Bindung und Solidaritätsempfinden können gemeinsame kulturelle Symbole sein. Gemeinsame Norm und Wertsetzung wie auch eine Tendenz zur biologischen Reproduktion innerhalb der Gruppe gehören dazu. Auf all dies sollte lediglich als ethnische Ressourcen geschaut werden, die aber nicht gleichwertig nebeneinander aufgefunden werden müssen, damit von Ethnie gesprochen werden kann (Haller 2005: 95).

Eine Ethnie wird vor allem generationenübergreifend gedacht, was das Abstammungskriterium erklärt, denn die Ethnie umfasst sowohl lebende, wie tote und künftige Individuen und bietet damit leicht nachempfindbare Kontinuität in Bezug auf das Gruppenzugehörigkeitsgefühl, die die persönlich erlebbare Dauer weit überschreitet. Es werden gar ethnische Kontinuitäten geglaubt, wo persönlich massenhaft Brüche erlebt werden (Reiterer 1998: 18-21). Das Bedürfnis nach historischer Kontinuität ist wichtiger Bestandteil von Ethnizität und gleichzeitig auch Grundlage für das von Eric Hobsbawm beschriebene Phänomen der Erfindung von Tradition für die Herstellung dieses Aspektes vom Wir-Gefühl. ${ }^{9}$ Anlehnend an Barth sollte berücksichtigt werden, dass Fremdzuschreibung einer Symbolisierung von Zugehörigkeit bedarf. Insofern Symbole immer verschieden deutbar, also multivokal sind, können sie die Integration verschiedener Meinungen der eigenen Gruppe gewährleisten und somit eine gewisse Konsistenz nach Außen darstellen.

„Symbolisations of community are umbrellas under which diversity can flourish, masks behind which a considerable degree of heterogeneity is possible. (...) This is always symbolised: in language, but also potentially in other forms, whether visual, musical or whatever.(...) This definitively relational nature of boundaries of identification is closely connected to the symbolisation of identity. In the first place, symbols only 'make sense in relation to other symbols. Meaning is a product of system and relation; nothing means anything on its own." (Jenkins 2008: 137-142).

\footnotetext{
${ }^{9}$ Hobsbawm führte zusammen mit Terence Ranger Erfundene Tradition im Umgang mit Geschichtsbewusstsein ein. Diese seien Fiktionen, die suggerierten, ein Symbol sei schon seit langer Zeit Tradition in einer Gruppe, obgleich die Nachforschung meist einen jüngeren Ursprung belegt (vgl. 1992 The Invention of Tradition. Cambridge: Cambridge University Press).
} 
Symbole können und sollen interethnisch verstanden werden. Daher sind ethnische Symbole häufig besonders gut sichtbar. Aber auch Vorstellungen von Biologie und Körperlichkeit können ethnische Aushängeschilder sein und stark variieren. Nicht nur emische Vorstellungen von gut sichtbaren Körpermerkmalen oder auch Schmuck, sondern auch von gemeinsamem Blut, Schwangerschaft und Krankheit können ethnische Marker sein (Schlee 2006: 80-88).

Auch wenn häufig Sprache als wesentlichstes der Kriterien für die Fremd- und Selbstwahrnehmung dienen mag, so machen doch Beispiele deutlich, dass auch dieses Kriterium oft erst konstruiert werden muss. Schaut man nur auf die deutschen Minderheiten in osteuropäischen Ländern, die sich mit politischen Forderungen zusammenschließen, greifen dabei keineswegs über weitreichende Sprachkenntnisse sondern vielmehr oft nur auf den Verweis eines deutschen Ahnen zurück.

Sofern sie nicht übergeordnete Kollektive ausweist, kann Religion ein gemeinsamer Marker sein, da sie ein Schwergewicht in der Alltagssinnstiftung spielt. Regelmäßig entstehen bei der Berührung ethnischer und religiöser Identitäten Synkretismen, die aufgrund ihrer Eigenart ethnisch integrierend wirken können. Zudem stiften religiöse Rituale Integrationsprozesse, die über den Verwandtenkreis hinausgehen und ein dauerhaft kollektives Gedächtnis anlegen können. Deutlich macht die Relevanz auch ein Blick zurück ins feudale Europa. Hier waren ethnische Vereinheitlichungsprozesse oft gekennzeichnet als Religionskämpfe, was sich erst durch die Rationalisierung auch von Identitätsstiftung und politischer Mündigkeit durch die Reformation wandelte. Religion dient wie Kultur oft der Legitimierung von politischen Konfliktparteien, wie sich beispielsweise der klassische Imperialismus christlicher Missionsideen bediente (Reiterer 1998: 130-132).

Raum und territorialer Bezug als ethnische Marker sind naheliegend, da Raum auch immer Residenz ist, der Ort, der Ressourcen bietet und das soziale Umfeld in der Regel beherbergt. Selbst wenn Globalisierungsprozesse und vermehrte Migration Raum nicht mehr nur real sondern auch idealisiert thematisiert, bleiben geographische Bezeichnungen und Benennungen eines Bezugsraumes, einer Heimat, für ethnische Identität von Bedeutung.

Boden, Religion und ethnische Identität hingen vielfach historisch belegbar zusammen. Anschaulich kommt dies in Termini wie das gelobte Land oder der synonymen Verwendung von Erde für Heimat und göttlich zugewiesenem Boden in semitischen Sprachen zum Tragen. Abschließend stütze ich mich auf folgende Definition, die Eriksen anknüpfend an Barth skizzierte: „Ethnicity is a relationship between two or several groups, not a property of a group.(...) Ethnicity is thus relational, and also situational: the ethnic character of a social encounter is contingent on the situation. " (2002:58). 
Hierbei sollte jedoch auch berücksichtigt werden, dass obwohl von ihrer Konstruiertheit ausgegangen wird, Ethnien keineswegs augenscheinlich imaginierte Gruppen sind, noch die ethnischen Marker beliebig und austauschbar wären. Sie verfügen tatsächlich wie auch die konstruierten Grenzen des Nationalstaates über erstaunliche Beharrlichkeit. Symbole sind oft emotional besetzt und vielfach langfristig im kollektiven Gedächtnis gefestigt. „Ethnische Gruppen sind damit zugleich sozial-kulturelle Wirklichkeit und Wirklichkeit als Konstrukt.“ (Heckmann 1997: 52). Für die Analyse von Ethnizität bedeutet dies vor allem die Stärkung des Blickes auf die Prozesshaftigkeit und Kontexte von Abgrenzungsmechanismen.

Interessant für aktuelle Forschungen muss sein, dass ethnische Sinnwelt heute aber vor allem mit anderen sozialen Identifikationsmöglichkeiten konkurriert und dementsprechend auch politisiert verstanden werden kann. So vermutet O'Shea für das zu untersuchende Beispiel: „, The existence and elaboration of Kurdish ethnicity is a vital strand of the Kurdish nationalist discourse, but ethnicity is both seen as given and used as a political tool rather than the cultural choice it could be." (2003: 29).

$\mathrm{Zu}$ der aktuellen politischen Dimension von Ethnizität gehört auch der Begriff Multikulturalismus. Er meint eine politische Zielsetzung, denn ethnischen Pluralismus hat es seit jeher gegeben. Als Ideal eines politischen Diskurses bemüht er sich um eine Abkehr von Assimilationskonzepten, die an einen ethnischen Nationalismus angeknüpft sind und die vor allem im 20. Jahrhundert zu Genoziden geführt haben (Ascherson 2004: 86f.).

Das Verhältnis von Ethnien und Nationen, das im Folgenden aufzuzeigen sein wird, ist nicht problemlos zu skizzieren. Denn bei teilweise sehr enger Verwebungen sind sie doch als von einander unterscheidbare Kollektividentitäten zu erfassen, begreift man Nation als politische Struktur, die das Verhältnis von Gesellschaft und Staat beschreibt. Deutlich wird die Differenz vor allem am Vorhandensein nationaler ethnischer Minderheiten oder dem multiethnischen Charakter vieler Nationalstaaten (Reiterer 1998: 13-16).

\section{Von der Nation zu internationaler Staatlichkeit}

„, Jede Nation spottet über die anderen, und alle haben Recht, “

sagte Philosoph Schopenhauer (2004: 204). Hierin schon wird deutlich, dass Nation keineswegs nur die Benennung einer politischen Struktur ist und gleichzeitig weist es schon auf die Notwendigkeit der Abgrenzung eines solchen sozialen wie funktionalen Gebildes zu anderen hin. Menschen leben in Nationen, sie sind Nationen.

Nation hüllt sich in ein vielschichtiges Bedeutungsgewand. Im Kontext der französischen Revolution war sie Slogan liberaler Republikaner, sie war Liebesobjekt der deutschen 
Romantik und wird heute unter Anderem schlicht als Ergebnis veränderter Sozialstrukturen infolge der Industrialisierung gesehen. ${ }^{10}$

Hobsbawm skizzierte phantasievoll in der Einleitung über Nationalismus das zeitgenössische Dilemma im Umgang mit Nation. Ihm nach wäre es einem Außerirdischen, der die Welt nach einem Atomkrieg vorfände, unmöglich die Geschichte der Menschen der letzten beiden Jahrhunderte zu verstehen ohne Kenntnis über den Begriff Nation. Jenem würde es vermutlich jedoch leichter fallen den Begriff zu beschreiben als uns Erdenbewohnern heute (2005: 11f.). Idealtypisch wird eine Nation verstanden als eine Gemeinschaft, die fundamental demokratisch ist, die ungeachtet ständischer Gliederungen jeden in gleicher Weise einbezieht. Sie sollte aus einer vorstaatlichen Gemeinschaft gewachsen sein. Sie soll die egozentrischen Kosten-Nutzen-Kalkulationen einzelner Wirtschaftsobjekte im Sinne der Vergemeinschaftung Max Webers zugunsten nationaler Solidarität überwinden (Estel 1997: 74). Die Ethnologie begreift Nation heute als ,eine gedachte Ordnung mit reziproken Verpflichtungen und familienerfassenden Zugehörigkeitsregeln, die einen überzeitlichen Charakter beansprucht und auf einen vorhandenen oder erstrebten Staat hin orientiert ist. “ (Elwert 2005: 267).

\subsection{Die verwirklichte Nation und Nation-Building}

Insofern die Rede von Nationalstaatsgründungen sein wird, soll vorab aufgezeigt werden, dass sich das Verständnis dieses sozio-politischen Prozesses in verschiedene Phasen unterteilen lässt. Zwischen den anfänglichen Ideen zur Nation und dem Aufbau der ersten Nationalstaaten bis hin zur Sicherung der weltweiten Stabilität durch die fremderwirkte Errichtung von Nationalstaaten liegen etwas mehr als 200 Jahre.

\subsubsection{Nationalismus: Katalysator für westliches Nation-Building}

„Nation-building is conceived as moving toward an ideal goal, set and rationalized by an ideology (or rather political myth) called „,nationalism “ of which several varieties can be distinguished." (Francis 1968: 339).

Nationalismus steht im Dienste der Gründung und des Baus von Nationalstaaten. Ihm geht die Forderung nach Souveränität als einem kollektiven Gut voraus (Hechter 2000: 11 und 125f.). Daneben ist er auf bestehende soziale Strukturen angewiesen, die solch kollektives Handeln ermöglichen. Angelehnt an Hobsbawm ist Nationalismus vor allem ein Prinzip, das fordert,

\footnotetext{
${ }^{10}$ Nicht nur im alltäglichen Sprachgebrauch sondern auch in der wissenschaftlichen Literatur besonders beim Rückgriff auf unterschiedliche sozialwissenschaftliche Disziplinen werden die Begriffe Nation, Staat und Nationalstaat synonym verwendet. In der Regel nehmen sie Bezug auf den nach westlichem Vorbild und den Vorgaben der Verträge der Vereinten Nationen entsprechenden modernen Nationalstaat, dessen Bevölkerung als Kollektiv mit gemeinsamer sozialer Identität gedacht wird. Ich möchte mich daher auf keine einzelne Definition oder künstlich geschaffenen Abgrenzungen stützen, sondern interdisziplinär angelegt, kritisch mit dem Begriffskomplex als einer historischen Idee auseinandersetzen.
} 
dass politische und nationale Einheit deckungsgleich sind. Zum Konstrukt der Nation schließe ich mich seiner Annahme an, dass sie

„eine gesellschaftliche Einheit nur insofern [ist], als sie sich auf eine bestimmte Form des modernen Territorialstaates, auf den "Nationalstaat" [bezieht], und es ist sinnlos, von Nation und Nationalität zu sprechen, wenn diese Beziehung nicht mitgemeint ist. “ (2005: 20f.).

Der durch Nationalismus in Gang gesetzte Prozess des Nation-Building ist eine Entwicklung, die mittels politischer, sozialer und ökonomischer Integration auf gesellschaftlich gehobener Ebene auf dem Territorium einer Nation wirkt (Friedmann 1967: 120). Die Genese des modernen Nationalstaats, der in Europa die feudalen, absolutistischen Monarchien ablöste, ist ein solcher Prozess, bei dem

„, die traditionellen Bezugs-Einheiten, wennschon nicht zerschlagen, so doch weitgehend ihrer normativen und integrativen Funktion entkleidet [werden]. Die lokale Gemeinschaft verliert ihren Gesellschaftscharakter, hört auf, ,terminale Einheit“" zu sein. “ (Reiterer 1998: 14).

Der Vertrag von Versailles hielt bereits fest, dass nationale Selbstbestimmung die bestehende Regierungsform ablösen solle. Es galt in den USA und Frankreich die Formel: Nation ist gleich Staat ist gleich Volk. Man ging von einem souveränen Volk aus, das als Nation nun an die zunehmend territorial verfasste Staatsform gebunden war, wobei gleichzeitig deutlich wurde, dass dies eine Vielzahl derartig verfasster Nationalstaaten hervorbringen würde. Hieraus ergeben sich aber keineswegs deutliche Kriterien oder Merkmale für Nation und nationale Zugehörigkeit, die über die territoriale Bindung hinausgehen. Gerade der zentralen Geburtsstätte des Nationenbegriffs, dem Einwanderungsland USA, musste es fern liegen, ethnische oder sprachliche Einheit als Marker zu deklarieren (Hobsbawm 2005: 31f.). Doch entwickelten sich „romantische“ homogenisierende Kriterien zu einer Zeit, in der keineswegs klar war, ob Hannover oder Holland es zu einer unabhängigen Nation bringen würden.

Die Ausgestaltung westlicher Nationalstaaten fiel überwiegend den politischen Eliten zu, wobei sich auch verschiedene Typen von Antinationalismen entwickelten. Der deutsche Föderalismus ist ein beispielhaftes Ergebnis solcher Parallelentwicklung.

Grund dafür, dass außereuropäische Ethnien lange Zeit als Völker und nicht als Nationen determiniert wurden, mag in erster Linie an der kolonialen Situation gelegen haben und daran, dass tribale Strukturen nicht als politisch anerkannt wurden (Francis 1968: 342). Mangelnde Gleichberechtigung und Hierarchisierung politischer Organisationsformen führten zu der verstärkten Einforderung seitens dekolonialisierter Staaten nach Souveränität, dem Recht seine Zukunft selbst zu gestalten. Die Selbstbestimmung als fundamentales Recht einer Nation, verankert in der Charta der Vereinten Nationen, legitimiert dies (Hechter 2000: 113).

Der Blickwechsel auf die Genese von Nationalstaaten im außereuropäischen Kontext erfasst entsprechend, dass zwischen postkolonialen und nicht kolonialisierten Staaten unterschieden 
werden muss. Letztere wie beispielsweise Iran fanden -mehr oder weniger- eigenständig den Weg in die internationale Staatengemeinschaft. Für den Irak wie auch viele andere Staaten im Mittleren Osten und in Afrika sollte berücksichtigt werden: „Colonialism had left intact political units that had adapted the functions and character of tribal authorities to the needs of rational administration. “ (Francis 1968: 343).

Koloniale Gesellschaften erlebten neue Repräsentationsformen von Eliten und Kollektivität sowie sie gleichzeitig unter dem Eindruck politischer Ideologien standen.

Obgleich hier kein evolutionistischer Ansatz vertreten wird, bleibt doch festzuhalten, dass der moderne Nationalstaat zumindest von der Entwicklung von Kommunikations- und Informationsmedien abhängig ist, die ermöglichen überregionale Strukturen zu schaffen und $\mathrm{zu}$ erhalten. Massenmedien kreieren neue Gruppen von Rezipienten und neue Imaginationswelten. So dienen moderne Technologien direkter Regierbarkeit (Hechter 2000: 26). Das flexible und unvorhersehbare Zusammenspiel von massenmedial verbreiteten Ereignissen und Migrationsprozessen wirkt darüber hinaus auch als Bindeglied zwischen Globalisierung und Moderne (Appadurai 1996: 4).

Infolge des Endes des Ost-West-Konfliktes traten seit den 1990er Jahren verstärkt regionale Konflikte in den Vordergrund. Ethnische Konflikte oder sogenannte Failed States tauchten als Krisenursache auf. Dass Staatszerfall und gesellschaftliche Fragmentierung für die internationale Staatengemeinschaft zunehmend problematischer und auch zu Quellen des internationalen Terrorismus werden könnten, zeichnete sich ab. Nation-Building, das in der Folge dessen betrieben werden soll, bezeichnet Politik, die ,ein Scharnier zwischen Außenund Entwicklungs- und Militärpolitik [bildet], das Gewaltkonflikten vorbeugen oder sie bearbeiten, lokale und regionale Stabilität erreichen und Entwicklung ermöglichen soll." (Hippler 2004: 15).

Diese Form des Nation-Building ist eine Neuerung vor allem insofern sie die bislang geachtete staatliche Souveränität missachten lernt. Einige Autoren gehen davon aus, dass der fremdgestaltete Prozess des Nation-Building nicht erfolgreich sein kann. Skeptisch blickt man vielfach auf Demokratisierung, welche in den Regionen, in denen vordergründig Wiederaufbau gewährleistet werden muss, schwer zu realisieren scheint.

Für die Zukunft wird vielfach ein Verlust der Bedeutung von Nation (nicht von Ethnizität) erwartet. So nimmt beispielsweise auch Hobsbawm an, dass

„Nationalstaaten“ und „Nationen“ oder ethnisch-sprachliche Gruppen mit der übernationalen Neuordnung der Welt zu kämpfen haben [werden], sie werden sich von ihr zurückziehen, ihr Widerstand leisten, sich ihr anpassen, von ihr absorbiert oder erschüttert werden. Nationen und Nationalismen wird es auch in Zukunft geben, aber in der Geschichte werden sie von untergeordneter und vielfach sehr geringerer Bedeutung sein. " (2005: 220). 


\subsubsection{Nationalismus und Kapitalismus}

„Damit eine Ethnie oder eine Gruppe von Ethnien eine Nation wird, braucht es den modernen Staat als dominante politische Struktur. Unter ,, modernem “ Staat ist ein Staat auf der institutionellen Grundlage einer unpersönlichen Bürokratie sowie eines kapitalistischen Weltsystems zu verstehen. " (Reiterer 1998: 237).

Dass die kapitalistische Entwicklung parallel $\mathrm{zu}$ dem explosiven Ausbreiten von Nationalstaaten in der Welt verlief, mag mehr als Zufall sein und daher als gekoppelte Ideologie erkannt werden. Im 19. Jahrhundert plötzlich war nicht nur die Rede von Nationen sondern auch von Volkswirtschaften. Gegenüber den früher regional begrenzten merkantilen Handelsformen wurde Kapital Nationen zugeschrieben und Handel zwischen Nationen und nicht mehr nur Einzelunternehmen betrieben (Hobsbawm 2005: 35).

„Die Existenz von Staaten mit einem Währungsmonopol und einem öffentlichen Finanzwesen
und somit auch fiskalpolitischen Aktivitäten und Maßnahmen war eine Tatsache. (...)
Darüber hinaus konnten selbst extreme Anhänger eines wirtschaftlichen Individualismus mit
Molinari akzeptieren, dass , die Aufteilung der Menschheit in autonome Nationen im
wesentlichen eine wirtschaftliche“ war (Molinari 1854 in Lalor 1889, Bd.2, S.957). Denn der
Staat - in der nachrevolutionären Ära der Nationalstaaten garantierte schließlich die
Sicherheit des Eigentums und die Einhaltung von Verträgen. (Hobsbawm 2005: 40).

Während die Verbindung von Wirtschaft und Nationalstaatlichkeit im 19.Jahrhundert historisch wuchs, war das 20. Jahrhundert geprägt von nationalen Bewegungen, die in erster Linie der Schaffung von Unabhängigkeit von imperialer Verwaltung dienten. Nationale Befreiungsbewegungen in ehemaligen Kolonien orientierten sich an der Theorie des westlichen Nationalismus, erschufen der westlichen Realität entsprechend ethnisch und sprachlich heterogene Staatsgebilde, die in der Lage sein würden am weltwirtschaftlichen Geschehen teilzuhaben (Hobsbawm 2005: 193-194). Nation-Building war Mitte des 20.Jahrhunderts eingebettet in Modernisierungstheorien und stand auf der politischen Agenda als Methode Gesellschaften strukturell den Industriestaaten anzugleichen. Der Begriff geriet in Zeiten des Protestes gegen den Vietnam-Krieg etwas in Vergessenheit (Hippler 2004: 16f.). Die Notwendigkeit in Entwicklungsregionen nationalstaatliche Strukturen zu bilden wird deutlicher, stehen die Bewohner erst global agierenden Großkonzernen gegenüber.

Revolution im Transport- und Kommunikationswesen führen aber derweil auch zum Verlust der Bedeutung von Nationalstaaten, wie der Anstieg nichtstaatlicher internationaler Organisationen von 831 im Jahr 1951 auf 4615 in 1984 belegt. Im ökonomischen Sektor stellen gemeinsam kontrollierte Gremien wie der internationale Währungsfont neue Ansprüche an Nationalstaaten (Hobsbawm 2005: 209). Durch Abkopplung von den nationalen Ökonomien verlagern sich Wertschöpfung und Wertrealisierung zunehmend in den globalen Raum, der übergeordnete Normen, Strukturen und Institutionen generiert. Seit den 
1990er Jahren sahen neben linken Politikern auch Sozialwissenschaftler die Gefahr des systemschädigenden Kapitalismus, wie er sich als Weltwirtschaftskrise in 2009 äußerte.

„,Wird der demokratische Staat als jenes notwendige Instrument betrachtet, welches historisch in der Lage war, den aufstrebenden liberalen Kapitalismus zu bändigen und produktiv zu nutzen, so bahnt sich gegenwärtig ein Bruch dieses Prinzips an. Im globalen Raum fehlen die demokratischen Instrumente, welche notwendig wären, um den ausbreitenden Wirtschaftsliberalismus in weltweite wohlfahrtsstaatliche Bahnen zu lenken. "

(Wicker 1998: 17).

\subsection{Nationale Identität}

Insofern ich in dieser Arbeit annehme, bestimmte Marker bei den Kurden als ethnisch konstitutiv ausmachen zu können, bleibt der Frage nachzugehen, inwieweit sich auch nationale Identität belegen und mithilfe bestimmter Kriterien erfragen lassen könnte.

Vermeintlich dem Menschen allgemein zugrundeliegende psychische Gegebenheiten, die sowohl ethnische wie nationale Identität provozierten, sind mit Vorsicht zu bewerten. ${ }^{11}$

Gerade im in dieser Arbeit nachzuzeichnenden Fall der Kurden, vielfach als „Volk ohne Staat" bezeichnet, erscheint die Abwesenheit eines gemeinsamen Nationalstaates, des legitimen Mittels für die politische Selbstbestimmung als Identitätsmangel.

Entsprechend der konstruktivistischen Betrachtung von Ethnizität ${ }^{12}$ wird von SozialwissenschaftlerInnen zu Nationalität festgehalten:

„, The idea that nationality is necessarily owed to the existence of objective differentiae such as language and religion had faded. None of these tangible group characteristics necessarily produces national solidarity: language is constitutive of national identity in some places but not in others, religion is constitutive of national identity in some places but not in others, and so forth. Nowadays it is fashionable to insist in the intersubjectivity of nationality. Rather than the deterministic outcome of social conditions, nations are increasingly regarded as entities that are socially constructed." (Hechter 2000: 13).

Was die nationale Gesellschaft liefert, ist der Handlungsrahmen einer demokratiepolitischen Einheit, die eingebunden in viele multilaterale Beziehungsnetze heute unverzichtbar erscheint und bereits deswegen identitätsstiftend sein mag. Nach den kollektiv bindenden Kriterien, die für das Erfassen von Ethnien galten, wird in Bezug auf den Nationalcharakter gefragt.

Zunächst ist vordergründig die Verbindung von Raum und Kollektiv gegeben. Im Nationalstaat wurde das Heimatbewusstsein auf eine größere Struktur übertragen, die die

${ }^{11}$ Zwar ist der Rückgriff auf Freudianische Konzepte, die beispielsweise das Es als kulturunspezifische menschliche Denkfigur anbieten, naheliegend, aber es gilt zu berücksichtigen, dass der Rückgriff auf Erklärungen der Psychoanalyse aufgrund der kulturspezifischen Orientierung und mit Blick auf die historische Hochphase des Nationalismus, in der sie als Disziplin entstanden ist, schwierig ist.

12 Hobsbawm hält diesbezüglich fest: „Erstens bieten offizielle Ideologien von Staaten und Bewegungen keine Anhaltspunkte für das, was in den Köpfen selbst ihrer loyalsten Bürger oder Anhänger vorgeht. Zweitens haben wir insbesondere keinen Grund zu der Annahme, dass für die meisten Menschen die Identifikation mit Nation - sofern sie existiert- alle anderen Identifikationen, die ein gesellschaftliches Wissen ausmachen, ausschließt oder ihnen immer oder überhaupt überlegen ist. Tatsächlich verbindet sie sich immer mit Identifikationen anderer Art, auch wenn sie diesen gegenüber als vorrangig empfunden wird. Und drittens kann eine nationale Identifikation samt allen ihren Weiterungen im Lauf der Zeit, ja selbst innerhalb sehr kurzer Perioden verändern und verlagern. “ (2005: 22). 
unmittelbar erlebte Lebenswelt überschreitet. Lokalität als Eigentum sozialen Lebens und kollektiver Identitätsstiftung schafft auch stets Nachbarschaft und nachbarschaftliche Beziehungen, was im weitesten Sinne auch in der Schaffung überstaatlicher Strukturen zum Ausdruck kommt (Appadurai 1996: 182).

Was die gemeinsame Zeiterfahrung angeht, so ist in der nationalen Gesellschaft die Schaffung kollektiven Gedächtnisses mittels Symbolträgern und symbolischen Ereignissen, an denen das Individuum selbst nicht partizipiert, kennzeichnend und stützt die These, dass gemeinsame Zeiterfahrung allgemein für Gruppenbildung bedeutend ist.

In Transformationsphasen wie dem Nation-Building dominieren oft Diskurse, in denen (ethnische) Homogenität verhandelt wird. Dabei kann Volkszugehörigkeit im Herderschen Sinn rhetorisch verklärt werden. Zu den jungen Beispielen zählen die stark überzeichneten ethnischen Homogenitäten in Verfassungstexten der früheren Sowjetstaaten, die sich um einen starken Bezug auf vorsozialistische gemeinsame Tradition und vermeintliche Geschichte stützen (Giordano 1997: 69f.). Trotz vieler Bestrebungen den Eindruck von Homogenität $\mathrm{zu}$ erzeugen, wurden tatsächlich im Zuge der Schaffung ökonomischer nationaler Großeinheiten Heterogenität bereitwillig hingenommen. Schließlich bestritt auch keiner die sprachliche oder ethnische Heterogenität in den anerkannten und ältesten Nationalstaaten wie Spanien oder England. Aus ökonomischen Gründen schien die Entmischung kleiner „Nationalitäten“ und kulturelle Normierung kaum sinnvoll. Schottland und Irland gingen in England als Nation auf, die Niederlande mit bescheidener Größe sonderte sich von Norddeutschen ab, was die Rolle der Mundarten gering erscheinen lässt. Erst die liberal ideologisierte Nation erforderte im politischen Sinne die Assimilation kleinerer Gemeinschaften und Völker (Hobsbawm 2005: 49-52).

Insofern sich der Nationalstaat als Souverän beschreibt und Verfügungsgewalt über die Existenz der Subjekte auf seinem Territorium hat, bedarf es nationaler Solidarität, wenn er nicht als Gewaltstaat in Erscheinung treten möchte. Gerade für sozialstaatliche Aspekte ist die legitime Solidarität ein wichtiger Bestandteil, eine sozialpsychologische Quelle für Teilen. Durkheim beschrieb, dass das Autonomwerden des Individuums zu einer stärkeren Abhängigkeit von der Gesellschaft infolge der Arbeitsteilung führe und eine Ausweitung des Solidaritätsbegriffs erfordere. Ausgenommen religiöse Gruppen stellen Nationen die größten Solidargemeinschaften dar. Aktuell wird dies besonders deutlich bei der Zusammenführung verschiedener Sozialpolitiken auf europäischer Ebene, wobei nationalstaatliche Akteure eine Zusammenlegung von Geldern und Zielen immer wieder zu Ungunsten eines gesamteuropäischen Projekts verhindern. 
Ganz allgemein wirken aktuell multikulturelle Gesellschaftsentwürfe und suprastaatliche Konzepte wie die Europäische Union auf nationale Identitätsstiftung. Indem die früher als natürlich und schicksalhaft empfundene nationale Zugehörigkeit einer unspezifischen Zustimmung zu Demokratie und Toleranz weicht und nationale Außengrenzen fließend werden, nimmt die Rückbindung an den Nationalstaat wie auch das Solidargefühl mit seiner Gemeinschaft ab (Estel 1997: 84). ${ }^{13}$

Weniger pessimistisch schätzt Hechter die Zukunft nationaler Identitäten ein. "This kind of social identity rests on distinctive national production and/or comsumption values. To the large extent, these values are nurtured by the stratification of culturally distinct groups, or by their capture of key institutional sectors. Since neither capitalism, socialism, nor any other post-industrial social formation has managed to eradicate cultural stratification, inspite of policies like affirmative action, and since immigration leads to ever new forms of it, this source of national identity is likely to remain with us in the foreseeable future." (200: 135).

Ein zentrales Instrument für die spezifische Herstellung nationaler Identität ist die Grenzziehung. Dieses Symbol schafft erst ein Bewusstsein der Bezugsgruppe im Nationalstaat, die persönlich nicht umfassend $\mathrm{zu}$ erfahren wäre. Je näher das Grenzbewusstsein und das Leben an den Grenzen aber bei einander liegen, umso größer ist die Wahrscheinlichkeit, dass diese Definition bröckeln kann. ${ }^{14}$

\subsection{Ethnische Konflikte und nationale Grenzen}

„Borders are both institutions and processes. As Institutions, they mark and delimit state sovereignty and rights of individual citizenship. As processes, borders have a number of functions. They are instruments of state policy, although the state's policies may be enhanced or impeded by the degree to which it exercises actual control over the border and its people. Anderson also recognises that borders are markers of identity, and have played a role in this century in making national identity the pre-eminent political identity of the modern state. This has made borders, and their related narratives of frontiers, indispensable elements in the construction of national cultures." (Donnan 1999: 5).

Ausgehend davon, dass Nation-Building als Strategie der Grenzerweiterung Ethnien aufeinandertreffen lässt, stelle ich nach Wimmer hierfür drei mögliche Typen vor. Der verbreiteteste Typ des Nation-Building fördert eine friedliche Assimilation an eine ethnische Kultur und Sprache der staatstragenden Elite. Der zweite Typ bemüht sich um eine neue Mixtur der Ethnien, die eine typisch neue nationale Identität fördern soll. Diese Kreolisierung gibt es ideell beispielsweise in Mexiko. Bei dem dritten Typ wird eine Führungsschicht

\footnotetext{
${ }^{13}$ Hierbei handelt es sich allerdings um ein Phänomen vor allem westlicher Industrienationen, in denen Sozialsystem und Gesellschaftsverträge an Stabilität verlieren, wohingegen diese Strukturen andernorts erst im Entstehen begriffen sind.

${ }^{14}$ Auf Anthropologie in Grenzgebieten werde ich nicht weiter eingehen, da es mir um die internen Prozesse geht. Die Aushandlungsprozesse in den Grenzgebieten sind gewiss jedoch mit Blick auf die Spaltung der Kurden ein sehr spannendes Thema wäre.
} 
kreiert, die sich nicht auf regionale oder ethnische Gruppierungen zu stützen braucht, die sich wie in der Schweiz. juristisch legitimiert (Wimmer 2006: 15f.).

Für die Darstellung interethnischer Beziehungen, die durch diese Typen von NationBuilding geschaffen werden, bietet die Ethnologie verschiedene Modelle an.

Haller fasst hierunter zunächst den Genozid. Dieser bezeichnet eine planmäßige Ermordung von Angehörigen einer Ethnie, während der Ethnozid den Versuch der Auslöschung identitätsstiftender ethnischer Merkmale und Symbole meint. Weiter nennt er Segregation und Differenzierung, die infolge von Unterdrückung oder Geboten zu getrennten Entwicklungen führen. Neben verschiedenen Formen der Assimilation kann Integration um die Anerkennung von Differenzen bemüht sein. Synkretismen sind Phänomene allgegenwärtiger kultureller Verschmelzungen, die aber auch umfassend ethnisch stattfinden können. Dank des Konzeptes der Hybridität von Homi Bhabha wird bei Synkretismen ungeachtet der Machtverteilung eine Veränderung auf beiden Seiten angenommen (2005: 97).

Die „Dramaturgie“ ethnischer Konflikte beschrieb Turner als liminales Ereignis, bei dem es zuerst zu einem Bruch infolge mangelnder Anerkennung der Sozialordnung und ihren Normen käme, worauf die Krise als kollektives Trauma folge, die geschlichtet werden und in die Reintegration münden müsse. Letzteres kann sowohl die Wiedereingliederung der Krisenauslöser wie auch eine konsequente Trennung sein (1974: 37-40). Sich in diesem Sinne Ereignendes wurde in den 1960er Jahren als Tribalismus bezeichnet und neuerdings vielfach mit den Begriffen Ethno-Nationalismus oder Separatismus beschrieben. Gemeint ist die Berufung auf eine bestimmte Ethnizität im Kontext der Einbindung in moderne Staatlichkeit.

Mehrere Autoren bezeichnen das letzte Drittel des 20. Jahrhunderts als das der separatistischen Bewegungen. Obwohl sie an die kleinstaatlichen Bewegungen, die sich beispielsweise gegen die Habsburger richteten, erinnern mögen, zeichnen sie sich doch insbesondere häufig durch eine sehr ablehnende Haltung gegenüber der modernen politischen Ordnung auf nationaler Ebene aus, die sich auf diffuse Ängste stützt.

\footnotetext{
„,Was solchen Abwehrreaktionen gegen wirkliche oder vermeintliche Bedrohungen Nahrung gibt, ist ein Zusammenspiel von internationalen Bevölkerungsbewegungen und den ungeheuer raschen, tiefgreifenden und beispielslosen sozioökonomischen Veränderungen, die für das dritte Viertel unseres Jahrhunderts so typisch waren. (...) Was haben solche ethnischnationalistischen Reaktionen überhaupt gemeinsam mit dem in letzter Zeit zu beobachtenden „Fundamentalismus" in vielen Teilen der Erde, von dem gesagt wird, er wende sich an Menschen, die keine Zufallsexistenz und keine unerklärten Zustände ertragen können und deshalb häufig denen zuströmen, die möglichst vollständige, allumfassende und phantastische Weltanschauungen anbieten?(...) Die Ähnlichkeiten mit einer Reihe von ethnisch/nationalistischen Phänomenen aus jüngster Zeit sind nicht zu übersehen, vor allem wo diese mit einem gruppenspezifischen religiösen Glauben verbunden sind oder solche Verbindungen wiederherzustellen versuchen.(...) Dennoch ist eine wichtige Unterscheidung zu treffen. Der Fundamentalismus, in welcher religiösen Gestalt auch immer, bietet ein
} 
detailliertes und konkretes Programm für einzelne Individuen wie für die Gesellschaft, selbst wenn dieses Texten und Traditionen entlehnt ist, deren Brauchbarkeit für das späte 20. Jahrhundert nicht ohne Weiteres auf der Hand liegt. Die angebotene Alternative zur gegenwärtig entarteten und bösen Gesellschaft stellt die Gläubigen vor keine unmittelbaren Probleme. (...) Die Berufung auf ethnische Zugehörigkeit oder eine Sprache ermöglicht keinerlei Orientierung für die Zukunft, auch wenn neue Staaten auf der Grundlage solcher Kriterien errichtet werden. Sie ist lediglich ein Protest gegen die anderen, die die ethnisch definierte Gruppe bedrohen. " (Hobsbawm 2005: 195-199.).

$\mathrm{Zu}$ dem, was unter anderem als Ethnisierung der Politik gilt, erklärt Wicker:

„Der Rückgriff auf essentielle Kategorien hilft den in solchen Bewegungen involvierten sozialen Akteuren, utilitaristische Motivationen und Ziele, welche implizit vorhanden sind, unausgesprochen zu lassen und allein jene Kategorien explizit werden zu lassen, welche Bezug auf das naturhaft Gegebene nehmen: Nationalisten handeln im Namen der Nation, die es aufrechtzuerhalten und zu verteidigen gilt, religiöse Führer im Namen der Religion und ethnic leaders im Namen der ethnischen Gruppe "(1998: 26).

$\mathrm{Zu}$ Regionalisierung sagt er: „Jene Bedeutungssteigerung, welche Regionen im ausgehenden Jahrhundert erfahren, die sich nur verstehen lässt vorm Hintergrund, dass Regionen zunehmend nationalstaatliche Interessen und Homogenisierungsansprüche kontrastieren, steht ohne Zweifel in enger Beziehung zu jenem tiefgreifenden Strukturwandel, der unter Einfluss der Entwicklung suprastaatlicher Organisationen und der Herausbildung von globalisierter wirtschaftlicher Konkurrenz erfolgt. "(1998: 30).

Unter Einbezug dieser Annahme erscheint die Analogie zu religiösen Konflikten, wie sie Reiterer macht, verständlich. Ihm nach entspräche die häufig schwer für Unbeteiligte nachzuvollziehende Brisanz ethnischer Konflikte den religiösen. Denn bei der emotionalen Einfärbung von ethnischen wie nationalen Symbolen ginge es schließlich um Identitäten, wo es in religiösen Konflikten um die obersten Wertsysteme ginge (1998: 23). Oppositionelle Führer oder Aktivisten suchen nach Unterstützung innerhalb der Ethnie, der sie sich selbst zuordnen. Sie appellieren an ethnische Solidarität, um ihre Anliegen als ethnisches Interesse darzustellen (Francis 1968: 344). Eine verbreitete These ist, dass Ressourcenverknappung politische Interessen schaffe, die mittels ideologisierter Gemeinschaftsvorstellungen umgesetzt werden sollen. ${ }^{15}$

Definitionen von Terrorismus in diesem Zusammenhang sind vielfältig. Kennzeichnend für nationalistischen Terrorismus, wie man ihn für Irland und die türkische Kurdenregion beschreibt, zeichnet sich dadurch aus, dass er in wirtschaftlich schwächeren Peripherien, in der die Zentralgewalt nur wenig ausrichtet, entsteht, wobei es eine enge Identifikation mit der eigenen Minderheitengruppe gibt und „Terroristen“ durch Generationenkontinuität in die Gruppe sozial eingebunden sind (Klugmann 2002: 18).

\footnotetext{
${ }^{15}$ Wicker meint diesbezüglich: „Sind Multikulturalismus und ethnische Konflikte eine Folge des härteren Wettbewerbs um knappe Ressourcen, drängt sich logischerweise die Sicht auf, dass künftige Entwicklungen, die auf marktwirtschaftlichliberaler Ethik fußen, ebenfalls die Multikulturalisierung und das Austragen von sozialen und regionalen Konflikten auf ethnischer Ebene vorantreiben werden. Politiker, die weniger Staat und mehr Wettbewerb fordern, tun deshalb gut daran, sich Überlegungen zur Prävention und Beilegung von ethnischen Konflikten zu machen. “ (1998: 61)
} 
Neuere, auch post-national bezeichnete Ansätze, die sich mit trans-staatlichen Räumen beschäftigen, überwinden national begrenzte Identitäten, indem sie eine Sichtweise kreieren, die Staatsbürgerschaften nicht mehr exklusiv an den Nationalstaat binden, obwohl dieser in der Regel wichtige Bürger- und Menschenrechte von Immigranten sichern kann.

„Inzwischen tolerieren über die Hälfte aller Staaten der Welt doppelte Staatsbürgerschaft. Diese Entwicklung berührt Kernfragen der Politik, so etwa das Gleichheitsprinzip und die Identitätsannahme in der Demokratie, Auffassungen individueller und kollektiver Identität und Loyalität von BürgerInnen untereinander und in ihrem Verhältnis zu Staaten. Sie berührt auch die oft deckungsgleich gedachten Konzepte „,Nation“ und Staat." (Faist 2000: 109f.).

Das Konzept des transnationalen Raums entwirft einen neuen sozial-relationalen Raumbegriff, der geografische Grenzen in ein neues Licht rückt, da er

„, die soziale Praxis von individuellen und kollektiven Akteuren in Territorien bzw. Orten - und dies sowohl in politischer, ökonomischer und kultureller Hinsicht [bezeichnet]. Der so verwendete Raumbegriff beinhaltet daher nicht allein physische Eigenschaften im Sinne traditionell geographischer Zielrichtung. " (Faist 2000: 114).

Wertfrei lässt sich festhalten, dass diese Handhabung den Begriff des Raumes für die Erfassung und Nutzung von Ethnizität wieder attraktiver gegenüber der nationalen Identität macht. Menschen, die in Diasporen leben, gelten nun als welche mit Hybrid-Identitäten (Faist 2000: 124). Nationale Identität dient demnach weniger der Identifikation als der Sicherung eines bestimmten Rechtsstatus. Der Nationalstaat ist um seiner Selbst Willen zu dieser Sicherung angewiesen, denn zur Verhinderung separatistischer Bestrebungen ist ein juristisches System und dessen Glaubwürdigkeit unabdingbar. Institutionen, die Rechte sichern, müssen beständig und glaubhaft von der Zentralgewalt betrieben werden, um Stabilität zu wahren (Hechter 2000: 152). Zum Schutz der ordnenden Funktion muss der Nationalstaat einen Weg im Umgang mit nachlassender Identifikation finden, die auch neonationalistische wie separatistische, ethnische wie religiöse Provokationen hervorrufen mag.

\subsection{Zur Politik des Nation-Building}

Auf die neuartigen Entwicklungen infolge des Endes der Blockkonfrontation wurde bereits hingewiesen. Dass Staatszerfall und gesellschaftliche Fragmentierung für die internationale Staatengemeinschaft zunehmend zum Sicherheitsproblem werden könnten, veränderte den Umgang mit Staatlichkeit sehr. Die Form des fremd erwirkten Nation-Building missachtet nicht nur staatliche Souveränität, indem sie sich Begriffen wie Peacekeeping bedient, sondern wird sogar als imperiales Nation-Building bezeichnet (Hippler 2004: 256).

Worum es nun gehen soll, ist die Tatsache, dass Nation-Building den betroffenen oder gestaltenden Subjekten stets nicht nur eine neue kollektive Identifikationsmöglichkeit bietet, sondern ein neues Politikverständnis mit einem sehr genauen Bild des politisch mündigen 
Bürgers mitliefert, was für Gruppenverständnis im Allgemeinen eine größere Rolle spielen dürfte als es bislang in ethnologischer Literatur berücksichtigt wurde, da es nicht nur Traditionen angreift, sondern auch „Verlierer“" schafft.

\begin{abstract}
„Nation-Building macht die Mitglieder einer Nation also prinzipiell zu politischen Subjekten, auch wenn in der Realität die Wahrnehmung partizipativer Rechte oft genug verweigert wird. Nation-Building politisiert die Bevölkerung zu einer Nation, es mobilisiert gerade im Konstituierungsprozess weite Teile der Gesellschaft.(...) Dies bedeutet allerdings, dass in der Gesellschaft zuvor schlummernde Konflikte, die durch den Ausschluss der Bevölkerung von der Politik nur geringe Artikulationsmöglichkeiten hatten, verstärkt wirksam werden können. Umso mehr wird dies zutreffen, wenn die Bestimmung dessen, wer eigentlich zur Nation gehört, ungeklärt oder umstritten ist, also insbesondere in multi-ethnischen oder multireligiösen Gesellschaften, die sich nicht auf gemeinsame Staatsbürgerschaft als Gemeinschaftskriterium einigen können. “ (Hippler 2004: 27).
\end{abstract}

\title{
3.4.1 Die Theorie des Demokratischen Friedens
}

Dass es ein politisches Interesse an der Konstitution von Nationalstaaten nach westlichem Vorbild gibt, ist kein Geheimnis und so sind Feststellungen, wie sie Dorman bereits 1980 machte, ${ }^{16}$ unter der Berücksichtung der politischen Theorie zu verstehen.

Ohne an dieser Stelle umfassend politische Theoriengeschichte nachzeichnen zu wollen, soll in Kürze die politikwissenschaftliche Theorie des Demokratischen Friedens dargestellt werden, insbesondere da sie globales politisches Handeln bestimmt. Die Theorie, die auf Kants Idee des Ewigen Friedens beruht, geht von einem Doppelbefund aus. Demnach führten erstens Demokratien gegeneinander keine Kriege, wobei sie zweitens dennoch nicht weniger konfliktgeneigt als andere Systeme seien. Insofern statistische Signifikanz die, oft als einzige unwiderlegbar bezeichnete, These stützt, bemühen sich verschiedene Ansätze um Erklärungen für das Phänomen. Diese reichen von sozial-konstruktivistischen, die annehmen, in Demokratien haben sich Normen und Ideen zur besseren Konfliktbewältigung durchgesetzt, bis hin zu strukturell-institutionalistischen, die das Abwenden von Krisen in der systemimmanenten schwerfälligen Entscheidungsfindung sehen. Für diesen Forschungszweig werden Statistiken geschaffen und Demokratisierungsgrad und bürgerliche Partizipationsmöglichkeiten in allen Staaten der Welt ermittelt und an den „vorbildlichen“ Daten der USA gemessen. ${ }^{17}$ Trotz Mangels an einer befriedigenden Erklärung für den Doppelbefund und wenigen Forschungsergebnissen über die Konfliktneigung junger Demokratien, gilt politisch das Dogma: Je mehr Demokratien es gibt, um so mehr Frieden muss es geben. Dies ist ein wissenschaftlich getragener Grundpfeiler nicht nur der US-

\footnotetext{
16 „The rise of a new ethnic consciousness was accompanied by a dramatic expression of interest both on the part of the academic community and the United States government. (...)The government granting agencies lent their generous support." (23)

${ }^{17}$ Hierzu zählen vor allem die Daten von Freedom House und Polity IV, die von US-amerikanischer Regierung finanziert werden, was sich bei einem Blick auf die Online-Präsenz schnell erkennen lässt.
} 
Außenpolitik, sondern ebenso verankert in den Zielen der Vereinten Nationen. Dass NationBuilding ein Aufeinanderprall von liberalen und realistischen Tendenzen schafft und Vorstellungen von der Wahrung der Sicherheit variieren können, findet dabei wenig Berücksichtigung. ${ }^{18}$ Komplikationen werden bisweilen durch unzureichende Demokratisierung erklärt (Geis 2001: 293). Probleme bei der darauf basierenden Politik treten immer stärker zutage.

„Nation-Building programs run into trouble when local actors do not endorse liberal norms. Reform becomes slower and more ineffective, prolonging the international presence in the target state and raising controversy over both the approach and appropriateness of nationbuilding. While the international objective is to enhance stability and peace for the benefit of all, local actors often seek to enhance power and security for their own benefit (...) The experience of nation-building shows that when the zone of peace and turmoil meet, turmoil is the likely winner unless international actors take affirmative steps to change this status quo. But taking those affirmative steps impinges on the principle of local authority, which is one of the important characteristics that distinguish contemporary Nation-building from the imperialism of the past." (Talentino 2005: 24).

\subsubsection{Strukturwandel und Institutionenbildung}

Größte Herausforderung für den modernen Nationalstaat ist es, einer größeren Mitgliederzahl den Eindruck von Kontinuität zu bieten. Während bestimmte Funktionen Subsystemen und übergeordneten Systemen übertragen werden, bedroht dieser strukturelle „Bruch“ seine Legitimität, denn auf strukturelle Diskontinuität wird dann mit ideologischer Kontinuität und ethnischer oder regionaler Loyalität reagiert. Europa ist durch die vorausgehende Verbindung ethnischer Loyalitäten eher ein Sonderfall der Staatengründung (Reiterer 1998: 241f.).

Die Vermittlung objektiver Strukturen, die Solidarität fördern und als Produkt gemeinsamer Zielsetzung egalitär bestehen, ist sowohl für komplexe wie auch kleinteilige Gesellschaften ein entscheidendes Erfolgskriterium. Das kann sich bei der Sicherung der Alltagsbedürfnisse auf lokaler Ebene wie der einer größeren Überlebenseinheit national ausdrücken. Der Integrationsmechanismus, sei er nun durch Subsidiarität oder unmittelbare Identifikationsmöglichkeiten hergestellt, verleiht erst den Strukturen und Repräsentationsformen Legitimation. Begreift man vereinfacht Ethnizität originär als abgegrenzte Abstammungsgemeinschaft, liefert sie beispielsweise als Clan totale Sozialstruktur. Die Ausdifferenzierung erfordert dann einen erweiterten Begriff von Gesellschaft, die sich in eine Anzahl aus Systemen mit unterschiedlicher Funktionslogik zerteilen lässt. Problematisch wird dabei, dass bei personal real oder fiktiv strukturierten Verwandtschaftsverbänden der Solidaritätsgedanke deutlich ist im Gegensatz zu dem in

\footnotetext{
${ }^{18}$ Für weiteres Interesse empfiehlt sich: Mansfield,E./ Snyder 1995: Democratization and War. Foreign Affairs, BD.74, Nr.3. 79-97 und Teusch U. /Kahl,M. 2001 Ein Theorem mit Verfallsdatum? Der Demokratische Frieden im Kontext der Globalsierung. Zeitschrift für Internationale Beziehungen, Bd. 2: 287-320.
} 
segmentären oder Großgesellschaften (Reiterer 1998: 81-90). Nation-Building ist also vor allem ein Prozess der Generierung neuer Strukturen, zu denen auch die Unterstellung unter Anti-Korruptionskonventionen und Weltbank zählt, das kollektive Zulassen einer kapitalistischen Wirtschaftsweise sowie eines demokratischen Politikverständnisses. Dieser Strukturwandel bedarf neuer Institutionen und den Aufbau von Bürokratie. Dieser beginnt jedoch nicht im luftleeren Raum. Gerade die orientalische Despotie verfügte bereits über einen weitreichenden bürokratischen Apparat. Wie bereits erwähnt, hinterließ der Kolonialismus funktionsfähige politische Strukturen, die den Ansprüchen in einer zunehmend ökonomisch globalisierten Welt am ehesten gerecht zu werden schienen. ${ }^{19}$

Institutionen dienen stets der Realisierung sozialer Verhältnisse, beispielsweise die Ehe. So sind sie auch Symbol. Im Nation-Building treten Parlamente an die Stelle von dynastischen Führerschaften. Es gilt eine bürgerlich-intellektuelle Schicht aufzubauen, die die vorigen Eliten einbindet oder sich neu konstituiert. Beide Schichten können sich auch gegenseitig instrumentalisieren und beispielsweise repräsentative Monarchien ausbilden. Wahlsysteme verändern politische Parteien, sie übersetzen Wahlstimmen in parlamentarische Präsenz, wobei politische Gruppen in den verschiedenen Systemen unterschiedlichste Repräsentanz generieren (Hechter 2000: 138). Das moderne Militär als Struktur einigt nicht nur gegenüber neu zu definierenden Kontrahenten, sondern offeriert auch verschiedenen Bevölkerungsgruppen eine neue Form gesellschaftlicher Partizipation (Reiterer 1998: 145).

Friedman nutzt in diesem Zusammenhang den Begriff der nationalen Performanz, welche im Kontext der Gesellschaftsstruktur, Besiedlungsdichte und ökonomischen Räume wirkt, indem sie Kerngebiete produziert, die im globalen Kontext als nationale Marker dienen. Regionen werden bestimmte Funktionen im Staat zugewiesen, Austausch zwischen den Regionen mittels neuer Verwaltungsinstanzen zentralistisch organisiert (1967: 123-125).

\subsubsection{Politische Eliten: Gewinner und Verlierer}

Großgesellschaften entwickeln derart auch zuständiges politisches Personal, eine politische Klasse, der sowohl Schutzansprüche geliefert und Rechtszusprechung abverlangt wird (Reiterer 1998 91). Hiermit wird aber auch einer Führungsschicht die Definition von Mitgliedschaft, die sich vorher meist primordial dargestellt hat, übertragen. Dies ermöglicht dem Individuum über die Zugehörigkeit zu reflektieren und sie rationalisiert als optional zu begreifen. Gerade Prozesse der Nationalisierung bedeuten ein Aufbrechen geschlossener lokaler und regionaler, politischer und sozialer Eliten und eröffnen neue Partizipations- und

\footnotetext{
${ }^{19}$ In welcher Weise hier Konkordanzmodelle, Einbindung kommunaler Repräsentation und Föderalismus
} diskutiert werden können, muss im Sinne der Fokussierung ethnischer Zugehörigkeit ausgelassen werden. 
Reflektionsmöglichkeiten. Ins Gewicht fallen sollte aber auch die Überlegung, dass politische Akteure zwar durch die Verdichtung von Symbolen zu Ritualen politische Realität schaffen, aber gleichzeitig sind sie auch Teil dieser Realität (Heidemann 2003: 166-170).

Ethnische Identität scheint auf einem Gleichheitsanspruch zu basieren, der sich auf Verteilungsgerechtigkeit stützt. Ethnische Konflikte in der Moderne beruhen und entwickeln sich oft dann, wenn unterschiedliche soziale Identitäten mit Verteilungsungerechtigkeit konfrontiert sind. Nicht ohne Weiteres $\mathrm{zu}$ beantworten bleibt, ob es in einer stark geschichteten Gesellschaft eine gemeinsame Ethnizität/Nationalität geben kann, wenn Teile andere Gesellschaftsgruppen dominieren.

Zum zeitgenössischen Konzept des Nation-Building gehören Verhandlungen mit und die Berücksichtigungen von lokalen Akteuren, denn „Imposition is not likely to be successful, especially if the resultng state does not correspond to what the local environment can support. (Talentino 2005: 25).

Nation-Building hat im Zuge der Errichtung neuer Strukturen immer Gewinner und Verlierer.

Das bedeutete auch, dass lokale Eliten instrumentalisiert und tribale Führungsschichten trotz neuer Strukturen erhalten werden können.

Francis stellte bereits die Hypothese auf ,, Tribal elites, having stake in the preservation of the status quo, are most likely to uphold ethnic values, and to legitimize their authority in ethnic terms, when challenged by non-tribal elites and by institutions of the nation-state. To the extent that they resist modernization they also reject nationalism. (...) A ruling or dominant elite is likely to legitimize its claim to power in ethnic terms than an elite in opposition.(...) An educated elite identifies with a particular ethnic society is likely to oppose the centralizing and homogenizing tendencies of the ruling national elite on ethnic groups, if the tribal elite identified with the same ethnic society admits members of the educated elite to key positions in sub-national (regional) institutions and to the ranks of the tribal elite itself." (1968: 346).

In ähnlicher Weise argumentiert Hechter: ,people who expect that their wealth, power, or prestige will increase from self-determination are those most likely to support nationalism.(...) As direct rule progresses, these people may deploy their resources to establish national movements in hope of retaining their position." (2000: 30).

Zusammenfassend möchte ich mit einem Zitat von Reiterer abschließen:

Soziale Identität konstituiert einen objektiven Sinnzusammenhang aufgrund gleicher bzw. komplementärer subjektiver Sinnzusammenhänge. Es gibt unterschiedlichste soziale Identitäten. In der ethnischen Identität charakterisiert der Bestandteil „ethnisch“ den gruppenorientierten Beziehungscharakter von Ethnizität. Ethnische Identität ist somit eine terminale soziale Systeme sowie umfassende (nichtdifferenzierte) Subsysteme herstellende Identität auf Grundlage der gleichen, d.h. austauschbaren Perspektive. Das kulturelle Element ist insoferne von Bedeutung, als und wenn die Beziehungen zwischen ethnischen Gruppen in Ausdrücken von Kulturdifferenz interpretiert werden. Diese Kulturunterschiede sind jedoch häufig von fiktiver Existenz, insbesondere in der Moderne, wenn Ethnizität die Funktion von Nationalität annimmt.“ ( 1998: 54). 


\section{Kurden im Irak}

„, The Kurds are the fourth largest ethnic group in the Middle East, and the largest without a state of their own. Still they exist in peripheral, underdeveloped parts of their host states. This very isolation has allowed them to maintain a separate identity even in the face of strong state centralization and hostility to their culture and political aspiration." (O'Shea 2003: 19).

Der Versuch Kurden allgemein als Ethnie zu kategorisieren, schließt sich an die zuvor dargestellte Definition von Ethnizität an. Ich habe darauf hingewiesen, dass sich Ethnien zwar über eine Fülle an gemeinsamen Merkmalen, für andere verständliche Marker, auszeichnen mögen, sich das Erstellen von Merkmallisten jedoch als unzureichend erwiesen hat. Daher werden nun einige wesentliche Marker des „Kurdentums“ vorgestellt mit dem Hinweis, dass dies nicht beanspruchen kann das gesamte Spektrum zu erfassen. Ein historischer Überblick wird den Kontext der zu analysierenden Gruppenbildungsprozesse deutlich machen.

$\mathrm{Zu}$ den Problemen der Kontextualisierung und Beschreibung des Kurdentums gehört, dass lange Zeit politische Definitionen die sozialwissenschaftlichen dominierten. Vor allem führte die Nationsgründung des Irak gemäß westlichem Verständnis zur Bewertung der „Iraker“ als einem homogenen Volk. Beispielsweise schrieb Craf 1944:

„The people of Iraq differ greatly from those of the English-speaking countries in their dress, customs, and religious beliefs. The people of Iraq are reserved friendly; (...) The native language of Iraq is Arabic. Many of the people speak French, Turkish and German, for all of those nations have had considerable influence on the population of this country."(1944: 305).

\section{Ethnische Marker des Kurdentums}

„Das zu beschreibende Phänomen [die kurdische Ethnie] aber existiert und schert sich wenig darum, dass die Wissenschaft es nicht in ihre Raster gepresst bekommt. " (Behrendt 1993: 39).

Anknüpfend an die Theorie und die Konflikte berücksichtigend, die vor allem um die ressourcenreichen Gebiete ausgetragen werden, findet zunächst der territoriale Bezug als ethnischem Marker Berücksichtigung, wobei auch die Konstruktion gemeinsamer Abkunft berührt wird. Da sich in ihrem gesamten Verbreitungsgebiet Kurden insbesondere durch sprachliche Unterscheidung „ausweisen“ und dies ein wichtiger Aspekt der Zurschaustellung ihrer Identität ist, wird Sprache an zweiter Stelle behandelt. Kultur zähle ich als Marker dazu, da mir Kurden in Gesprächen eben genau dies als Hauptunterscheidungsmerkmal zu Arabern, Türken oder Iranern beschrieben. Obwohl Kurden sich gerade nicht durch gemeinsame Religionszugehörigkeit auszeichnen, wird die religiöse Heterogenität vorgestellt, um einen Eindruck dieser konkurrierenden Identitäten zu vermitteln.

Kurden im Irak, der Türkei, Iran, Syrien und Aserbaidschan wie in der Diaspora gehören zum „Volk ohne Staat“, das selbst auf der Suche nach herausragenden Markern zu sein scheint. Es 
befindet sich im Wandel und muss nach einem Jahrhundert, beeinflusst von verschiedenen Ideologien und zahlreichen Konflikten, ein Selbstverständnis erlangen, das der äußeren Ansicht gerecht werden kann. Dies soll verdeutlichen, dass die nun folgende Auswahl lediglich als eine für diese Arbeit nützliche Selektion zu betrachten ist.

\subsection{Kurdistan: Der territoriale Bezug}

„, The area that can be generally described as Kurdistan consists of an arc of mountain chains enclosing a series of interior basins, astride the international boundaries of Iran, Iraq, Syria and Turkey(...) the entire area covers roughly $400000-450000 \mathrm{~km}^{2}$. Many peaks are higher than $4000 \mathrm{~m}$, and even most Kurdish cities are sited at over $1000 \mathrm{~m}$ above sea level.(...) The plains of Kurdistan have a sub-tropical climate, but the high elevation makes the winters very cold (...) Kurdistan is in essence land-locked. Its only access to the sea would be either overland or to the Persian Gulf via the Euphrate River.(...) Kurdistan's very peripherality meant that no imperial power regarded control of the region as absolutely necessary until after First World War, when both the discovery of mineral resources, and Kurdistan's challenges to state sovereignty, accorded the area a greater importance." (O'Shea 2003: 25).

Das hier zu behandelnde Irakisch-Kurdistan ist ein Teil des fiktiven Groß-Kurdistans, ein Teil des Gebiets, in dem Kurden sich beheimatet fühlen.

Der Ursprung des territorialen Bezugs der Kurden an das von ihnen heute bewohnte Gebiet ist schwer nachzuzeichnen. Diesbezüglich konkurrieren verschiedene Herkunfts- und Abstammungsmythen ${ }^{20}$ wie auch wissenschaftliche Auffassungen miteinander. Ob die Kurden ein Konglomerat von Einwanderern hauptsächlich iranischer Herkunft, Nachfahren der Meder, die im siebten Jahrhundert v.Chr. das Assyrische Reich eroberten, sind oder ob sie seit der frühesten Geschichte in dem Gebiet „verwurzelt“ sind, ist eine noch nicht zu beantwortende Frage (Asadi 2007: 22).

Vieles ist aus vorislamischer Zeit nicht bekannt, aber die weitreichende islamisch-arabische Eroberung, die sich im siebten Jahrhundert in Mesopotamien vollzog, machte auch Kurden zu Untertanen. Erst der innere Zerfall des Abbasiden-Kalifats gegen Ende des elften Jahrhunderts ließ kurdisch-islamische Dynastien Herrscher über einige Gebiete werden. Die Dynastie der Ayyubiden (1171-1252) des mystifizierten Saladins (Ṣalāḥ ad-Dīn), welche Ägypten und Syrien regierte, wie die Marwaniden (990-1096) zählten zu den wichtigsten Dynastien, die über Jahrzehnte hinweg jeweils große Teile des heutigen Kurdistan beherrschten (Asadi 2007: 23f.). Das heute auf mehrere Staaten verteilte Gebiet zwischen dem türkischen Taurusgebirge im Osten und dem westlichen Zagros Irans wurde erstmals namentlich, während der Herrschaft der Seldschuken im elften Jahrhundert dokumentiert. In den kommenden Jahrhunderten verhinderten wiederkehrende Einfällen anderer Gruppen und

\footnotetext{
${ }^{20}$ Im Schahname wird beispielsweise von einem König berichtet, der Kindern opfern ließ. Die durch einen Trick vor ihm Geretteten waren die Ahnen der Kurden. Je nach Region gibt es aber auch turkstämmige oder arische Ahnenmythen.
} 
die heterogene Gesellschaftsstruktur erfolgreiches imperiales Handeln einer lokalen Dynastie. Dies gelang erst wieder türkischen und iranischen Stämmen, die das safawidische und osmanische Großreich im 16. Jahrhundert hervorbringen konnten. Die Teilung der kurdischen Gebiete durch die Grenzziehung dieser Reiche bereitete die anhaltende Teilung in Form der iranisch-irakischen Grenze vor. Als Nomaden kannten Kurden weder feste Grenzen noch war Kurdistan als gedachte territoriale Einheit bekannt. Als Vasallen oder kleinere politische Einheiten der Osmanen existierten zu Beginn des 19. Jahrhunderts die drei mächtigen Dynastien: Soran mit der Hauptstadt Ruwandiz, Baban in der Sulaimani-Region und Badinan, dessen Regierungssitz in Amadia war. Innere Machtrivalitäten und wechselhafte Beziehungsgeflechte spielten der osmanischen Zentralgewalt in die Hand, die Mitte des 19. Jahrhunderts mit der Unterwerfung Babans alle kurdischen Zentren politisch neu ordnen konnte (Asadi 2007: 24-27).

Kurden leben und lebten in strategisch wichtigen Gebieten. Schon vor Entdeckung des Öls durchquerten Handelsrouten zwischen Asien und Europa die Region. Besonders die Tatsache, dass die wichtigsten Wasserquellen des Mittleren Ostens, insbesondere Tigris, Euphrat und Aras hier verlaufen, legt nahe, dass auch in Zukunft die Region bedeutsam bleiben wird.

Ohne der Darstellung verwandtschaftlicher Strukturen vorgreifen zu wollen, soll ein Vermerk Barths die Bedeutung von Boden für dörfliche Gemeinschaften hier deutlich machen:

"It should also be remembered that with the Kurdish type of lineage organization, the individual's loyalities are not to the descent group in abstracto, but are felt and expressed in terms of local groups, which very nearly correspond to the lineage segments. Because of this, any armed conflict between lineage segments will necessarily be in territorial terms; it will be a feud between villages." (1979: 76).

Die typische Wirtschaftsweise der bergigen Region, ebenfalls in einem späteren Teil der Arbeit behandelt, möchte ich nicht als exklusiven, ethnischen Marker hervorheben, denn „,weder tribale Organisation noch Wanderweidenwirtschaft sind also in dem Sinne spezifisch „,kurdisch“, als dass sie Kennzeichen einer einzigen homogenen Bevölkerungsgruppe seien, sondern es handelt sich um Lebens- und Wirtschaftsweisen, die sich in einem bestimmten naturräumlichen und historischen Umfeld als so erfolgreich erwiesen haben, dass Menschen der verschiedensten Religionen, Sprachen und Herkunft sie annahmen. " (Behrendt 1993:48).

Die von Genozid und Ethnozid betroffenen Kurden in der Türkei, dem Irak und den vier Provinzen Irans ${ }^{21}$ standen wegen ihres Lebensraumes oft im Zentrum wirtschaftlicher Interessen. Was den Irak betrifft so liegen hier wichtige Ölfelder um Mosul, Kirkuk, Khanaqin und Ain-Zaleh (vgl. Anhang VI).

\footnotetext{
${ }^{21}$ Eine dieser Provinzen trägt die Bezeichnung Kurdistan und ist nicht zu verwechseln mit der irakischen Autonomieregion.
} 
Von den 16 Provinzen, die der Irak seit der Monarchiegründung (1921) umfasste, zählten Sulaimaiya, Arbil, Dahuk und Kirkuk zu den kurdischen, die zuvor dem Welayat Mosul (vgl. Anhang I.a) zugerechnet worden waren. Die ersten drei bildeten später die Autonomieregion Irakisch-Kurdistan, wobei sich bereits vor Jahrhunderten Sulaimaniya als Zentrum der feudalen Baban-Administration etabliert hatte (Sharif 1991: 35). Problematisch bleiben bis heute die Grenzregionen Irakisch-Kurdistans, in denen kurdische Mehrheiten leben mögen, beispielsweise um Mosul oder Kirkuk, das unter US-Verwaltung steht. Artikel 2 der 2005 verabschiedeten Verfassung fordert eine Angliederung dieser Gebiete, die aus kurdischer Perspektive trotz heterogener Bevölkerung ein integraler Bestandteil Kurdistans seien. Araber leugnen diese Ansprüche. Christen fürchten hauptsächlich eine drohende Auseinandersetzung (International Crisis Group 2006: 3-6). Ein Zusammenschluss der von den geschätzten 30 Millionen Kurden bewohnten Gebiete Iraks wird auch von türkischer Seite abgelehnt und Volksabstimmungen werden regelmäßig aus Sicherheitsgründen verschoben. Beratende Wissenschaft rät aktuell: "All indications are that the Kurds cannot take Kirkuk, by law or by force, without triggering wide-scale violence." (International Crisis Group 2006: 29).

„,Strukturell besitzt der heutige Nordirak fast eine partielle Eigenstaatlichkeit, ist somit eine Art Konföderation als Gliedstaat eines gegenwärtig schwachen Staates. Das unter kurdischer Kontrolle stehende Gebiet verläuft wie ein halbmondförmiger Streifen entlang der türkischen und iranischen Grenze bis Khanaqin, stellenweise nur an die 30 km, in unwegsamen Gebieten bis zu 100 km breit." (Asadi 2007: 504).

\subsection{Kurdisch: Eine gemeinsame Sprache?}

Die Zuweisung einer Sprache zu einer gesellschaftlichen Minderheit bewirkt oft den Ausschluss aus öffentlichen Bereichen, wie der Bildung. Dessen SprecherInnen leben daher zumeist bilingual. Im politischen Kontext ist wesentlich, dass Sprache neben „Rasse“, Geschlecht und Religionszugehörigkeit $\mathrm{zu}$ den Bedingungen der Sicherung der Menschenrechte in der Charta der Vereinten Nationen zählt und den Minderheitenschutz ergänzt (Havrest 1998: 127). Das Kurdische findet dem Gedanken folgend in den verschiedenen Gründungsverträgen des irakischen Staats Berücksichtigung.

Die Sprache der Kurden als europäisches Forschungsobjekt wurde unter anderem erstmalig vom Göttinger Orientalist Michaelis (1717-1791) thematisiert. Dieser vermutete, dass Kurden Nachkommen der biblischen Syrer seien. In einer ersten Grammatik belegte der italienische Dominikanerpater Garzoni 1787 die Verwandtschaft des Kurdischen zum Persischen (Ağuiçenoğlu 1997: 175). Was die gemeinsame Abstammung betrifft, so erschweren auch Ereignisse wie das massenhafte „Konvertieren“ zum Islam und Kurdentum samt Sprachübernahme christlich-armenischer Dörfer im 19. Jahrhundert die Konstruktion 
(Behrendt 1993: 38). Die Meinungen über die kurdische Sprache gehen heute weit auseinander. ${ }^{22}$ Fest steht, dass sich das Kurdische trotz langer arabischer Herrschaft besser als das Türkische oder Persische gegen arabischen Einfluss wehren und seine Eigenständigkeit wahren konnte. Neue linguistische Studien in Abgleich mit dem Avesta belegen, dass das Kurdische zur nordwestiranischen Sprachgruppe gehört und selbst älter als das Persische ist, also kein Teil von ihr sein kann (Havrest 1998: 65). Gemeinhin gilt, dass das Kurdische eine eigenständige indogermanische Sprache ist, die sich in verschiedene, stark von einander abweichende Dialekte aufteilt (vgl. Anhang III.a). Uneinheitlich und komplex erscheint die klare Abgrenzung der Dialekte. Offenbar mangelt es an einheitlicher wissenschaftlicher Bestimmung von Dialekten. In der Regel zählt hierzu die regionale Geltung bei eingeschränkter kommunikativer Funktion gegenüber einer Einheitssprache. Eine der deutlichsten Dialektunterteilungen ist die des Kurmanji und Sorani. Viele Dialekte scheinen noch nicht ausreichend erforscht und werden erschwerend in den verschiedenen Staaten unterschiedlich bezeichnet. Darstellen möchte ich hier die Unterteilung nach Havrest 1995, die die Mundarten nach Unterschieden in der Phonetik und Formenlehre aufteilt, wobei auch hier die Grenzfälle nicht ausreichend berücksichtigt werden (vgl. Anhang III.b). Im Nordwesten dominiert das Kurmanji, in Zentralkurdistan bis Kermanshah das Sorani und im Süden das Gorani. Einige Mundarten wie Hawrami sind derzeit infolge politischer Unterdrückung oder wirtschaftlich begründeter Umsiedlungen vom Aussterben bedroht. Die angehängte Übersicht verdeutlicht, dass die Sprecher der zentralkurdischen Dialekte in Iran und Irak, sowie die Sprecher des nordkurdischen Dialekts untereinander gut verstehen (Havrest 1998: 83). Die von Kurden bewohnten Staaten handhabten den Umgang mit den Sprachen sehr unterschiedlich. In der Türkei waren meist kurdische Publikationen und Sprachförderung verboten, wohingegen der Iran aufgrund der sprachlichen Nähe in geringerem Maße gegen den Gebrauch vorging. Im Irak hing dies stark von dem Autonomiestatus ab. Sprachgesetze der Monarchie ließen bestimmte Dialekte in verschiedenen Regionen zu, wobei Kurden unter anderem in Bagdad wenig berücksichtigt wurden. Das Ende der Monarchie erhöhte die Hoffnung auf Besserung der Sprachpolitik, doch Kurden wurden zunehmend mit entsprechender Wirkung zu Staatsfeinden stilisiert. Die Zwangsumsiedlungen vieler Dörfer zerstückelten zusammenhängende Sprachgemeinschaften. (Havrest 1998: 174). Die jüngsten Entwicklungen in der Autonomieregion zeigen ein zunehmendes, staatlich gefördertes Interesse an der Sprache. Es finden Standardisierung und neue Wortschöpfungen statt, um Kurdisch als Amtssprache nutzen zu können.

\footnotetext{
${ }^{22}$ Sie sind vielfach von politischen, teilweise rassistischen Motiven geprägt, die sich bemühen das Kurdische beispielsweise als unterentwickelten Zweig des Persischen abzuwerten (Havrest 1998: 63-67).
} 
Lexika und Lehrbücher sind allerdings noch im Entstehen begriffen, was auch der starken oralen Orientierung der Sprache geschuldet sein mag. Dialektische Verschriftlichungen sind aber auch schon seit dem 15. Jahrhundert belegt, besonders als Poesie und Heldenepen.

Dem Kurdischen fehlt es nicht nur an einer gemeinsamen Hochsprache sondern einem einheitlichen Alphabet, denn es kennt im Irak, Syrien und Iran das arabisch-persische, in der Türkei und Armenien das Lateinische und Kyrillische (Ağuiçenoğlu 1997: 183).

\subsection{Kurdische Kultur}

Weder soll nun die Diskussion um „umkämpfte“ kurdische Kultur eröffnet, noch beansprucht werden alle Facetten der Kultur zu erfassen. Lediglich einige Aspekte, die sich am ethnologischen Kulturbegriff ${ }^{23}$ orientieren, sollen Erwähnung finden.

Was die schönen Künste angeht, so wurden diejenigen Kurden, die sich hier in besonderer Weise hervorhoben, zumeist von der dominanten Zentralgewalt absorbiert und sind der Weltöffentlichkeit entsprechend als Perser, Türken oder Araber bekannt. Des Weiteren sind Kernelemente der kurdischen Kultur selten in das Bewusstsein von Europäern vorgedrungen nicht nur wegen der mangelnden Kenntnis über Kurden, sondern auch da ein anderes Verständnis für Kunst und Kultur nötig ist. Dazu zählt besonders die orale Tradition vor allem im Kurmanji-Dialekt und bei den Yeziden. Mangelnde Verschriftlichung und Interesse an Gegenwartskultur haben zu nur wenig wissenschaftlicher Thematisierung geführt, obgleich diese Traditionen weit verbreitet und sehr kunstvoll ausgestaltet unter Kurden zu finden sind. Die bestimmten Formen der Performanz oraler Literatur sind neben der unzureichenden Erforschung zeitgleich durch zunehmende Verbreitung von Alphabetisierung und Neigung zu Verschriftlichungen bedroht. Niedergeschriebene Versionen des Kurdischen gab es zwar unlängst, doch waren diese vom heimischen Dialekt des Verfassers geprägt. „Die Bestrebungen kurdischer Intellektueller in Istanbul oder im Exil in Russland und Frankreich zur wissenschaftlichen Erarbeitung einer Nationalliteratur haben die breiten Volksschichten nur in geringem Maße. “ (Günther/Brentjes 2001: 23).

In Bezug auf wissenschaftliche Erkenntnis gilt Ähnliches für Musik und das kunstvolle Gestalten von Teppichen und Kostümen. Diese Aspekte zählt Kreyenbroek in dem einer Konferenz folgenden Sammelband zu kurdischer Kultur auf (1996: 2-6).

Was kulturspezifische Handlungen betrifft, so werden zahlreiche Rituale in kurdischen Dörfern durch die Derwischorden anlässlich vieler Feiertage ausgeführt. Sie beinhalten viele

\footnotetext{
${ }^{23}$ Dieser umfasst mehr als die sogenannten schönen Künste, nämlich neben Materiellem auch Handlungs- und Denkweisen, über dessen Bedeutung die Mitglieder einer bestimmten Gruppe übereinstimmen (Lang 2005: 220).
} 
Rites de passages wie Hochzeiten und Beschneidungen. Jährungen sind dabei eher unbedeutend, da Jahresangaben erst neuerdings vereinheitlich werden (Barth 1979: 111f.).

Kulturelle Symbolik ist eng verbunden mit Stammeszugehörigkeit. Diese lässt sich oft an den Kleidern der Männer, den Frisuren der Frauen oder auch an den Typen der Zelte erkennen, wobei diese Symbole wenig über den Status der Personen innerhalb des Stammes auszusagen vermögen (Yalçin-Heckmann 1991: 104).

\subsection{Religionszugehörigkeiten}

Unter Kurden herrscht religiöse Pluralität. So kann von islamischen (Sunniten und Schiiten), christlichen, jüdischen, yezidischen und anderen Kurden die Rede sein (vgl. Anhang IV.b). Auch die in Anatolien ansässigen Alewiten, die rund zehn Millionen Menschen umfassen, sind zum größten Teil Kurden (Günther/Brentjes 2001: 20).

Obgleich die Mehrheit kurdischer Stämme dem sunnitischen Islam, also der Orthodoxie, folgt, gibt es einen wesentlichen Unterschied zu den benachbarten arabischen Sunniten (vgl. Anhang IV.a), denn letztere sind überwiegend Anhänger der hanafitischen Rechtsschule, wohingegen Kurden eher der schafiitischen angehören (O'Shea 2003: 155). Schiitische Muslime, wie die Faili-Kurden, wurden in den Entwicklungen des letzten Jahrhunderts mehrfach in den Iran verwiesen. Daneben waren insbesondere im Irak bis zur Massenemigration nach Israel auch jüdische Kurden in etwa 146 Gemeinden zahlreich anzutreffen (O'Shea 2003: 29).

Eine gesellschaftliche Sonderrolle fällt den (sunnitischen) Derwischorden zu, bei denen die kurdische Hierarchie mit der islamischen Orthodoxie in Verbindung gebracht worden ist.

Die weit verbreitete Zugehörigkeit zu diesen Sufi-Orden konzentriert sich auf die Naqschbandiya, die auch in anliegenden Ländern eine wichtige Rolle einnimmt, und die Qadiriya, dessen Strukturen und Praktiken weitestgehend rein kurdisch sind (O'Shea 2003: 28). Die Verquickung von Islam und politischer Führung personifizierte als einer der Ersten Scheich Ubaidullah Nahri, der sich als Reaktion auf den politischen Zusammenbruch der kurdischen Emirate (erfolglos) bemühte unabhängige kurdische Institutionen $\mathrm{zu}$ formen (Asadi 2007: 26).

„Kurdistan is host to at least two religions of Kurdish origin. Like Alevism, both of these religions exhibit some superficial Islamic traits such as the veneration of Islamic figures: in the case of the Ahl-I-Haqq/Yaresan of Southern Kurdistan, the veneration of Ali, the Prophet Mohammed's son-in-law, and in the case of the Yezidis of Northern Iraq and eastern Turkey, veneration of Sheikh Àdi, a Sunni sufi. Both religions exhibit many features of Zoroastrian practices and beliefs, and there is reason to believe that they are both strongly influenced by an even earlier Indo-Iranian religion." (O'Shea 2003: 33). 
Yeziden sind besonders interessant, begreifen sie sich nicht nur als Ur-Kurden, sondern spielen auch eine zunehmend wichtigere Rolle in der Abgrenzung zu Muslimen. Ihr Heiligtum liegt in Lalesh in der Provinz Dohuk. Jedoch werden nur weniger als eine Million Anhänger im Nordirak vermutet und 80 Prozent außerhalb der Grenzen Kurdistans. Yeziden hatten sich trotz vieler feindlicher Umstände - denn Christen und Muslime bekämpften sie als Teufelsanbeter - ihre Religion regional bewahren können (Günther/Brentjes 2001: 20).

In einer Sonderausgabe der Zeitschrift Pogrom von 2007 werden neben Sunniten, Schiiten und Yeziden auch Ahl-e-Haqq, Assyro-Chaldäer und Neu-Christen, die durch den Zugang zu Bibelübersetzungen in den vergangenen Jahren konvertierten, zu den irakischen Kurden gezählt (Reinke 2007: 36f.). Ganz allgemein muss angemerkt werden, dass religiöse Identität im letzten Jahrhundert starke Konkurrenz durch säkular-nationale Ideologien hatte.

\section{Die Genese Irakisch-Kurdistans: Ein historischer Überblick}

„The Kurds have been largely sub-state actors, and as history is written by hegemons, Kurdistan's history is that of its occupiers. The early history of Kurdistan and the Kurds is either unrecorded or inadequate to form a clear narrative." (O'Shea 2003: 190).

Der folgende historische Überblick erfasst die Umstände, unter denen Kurden lebten und leben. Die Auswahl historischer, weitestgehend politischer Ereignisse, orientiert sich an den von mir angestellten Überlegungen zur Untersuchungsfrage.

Hinsichtlich der Beliebtheit imperialistischer Großreiche war das von Kurden bewohnte Gebiet schon im 6.Jahrhundert v.Chr. von Interesse für die Perser, die selbst 200 Jahre später dem Eroberungszug Alexanders unterlagen. Auf dem Gebiet tummelten sich Ansprüche von Griechen, Persern und Römern (Deschner 2003: 322). Kurden konnten sich seit Anbeginn islamisch-arabischer Dominanz im achten Jahrhundert, noch immer an Schnittstellen mehrerer Großreiche liegend, weitegehend autonomes Handeln erlauben und schwache Zeiten der Zentralmacht zu eigenem Aufstieg nutzen. Beispielhaft derartig sich entwickelnder kurdischer Herrschaftsdynastien sind die Marwaniden (990-1085). Die von ihnen verwaltete Bevölkerung war allerdings keineswegs homogen, noch sprach man am Hof Kurdisch. Die Marwaniden büßten ihren Einfluss durch den Anspruch auf Zentralgewalt der Seldschuken wieder ein (Behrendt 1993: 61). Infolge des Mongolensturms entwickelte sich die anarchische Region in Ermangelung eines Herrschers unabhängig bis sie im 16. Jahrhundert von den schiitischen Safawiden und später vom Osmanischen Reich unterworfen wurde. Den Pufferzoneneffekt nutzen Kurden geschickt, zumal es zu der Politik der Osmanen gehörte, die mächtigen Dynastien als Vasallenfürstentümer zu integrieren, sie autonom und unbesteuert zu belassen, um sie von einer Kooperation mit den Safawiden abzuhalten (Behrendt 1993: 100). 


\subsection{Die Ausgangssituation: Kurden vor dem ersten Weltkrieg}

Ab dem 19. Jahrhundert wurden Kurden in die Konflikte der orientalischen Großreiche mit europäischen Großmächten hineingezogen und von den Machtverwerfungen erfasst. Unter anderem hatte dies zur Folge, dass für die Aufstellung eines intakten Heeres, das vor allem Napoleons Streitkräften gewachsen sein sollte, die kurdischen Emirate unterworfen und der osmanischen Zentralmacht unterstellt wurden (Behrendt 1993: 154). Aufstände gegen diese Zentralgewalt erhoben sich in Baban, Soran, Bothan, und Hakkari (Özdemir 2006: 43). Visionen eines eigenen kurdischen Staates konkretisierten sich, nachdem auf den Grenzen als imaginären Interessensphären von einer persisch-britisch-russisch-türkischen Kommission 1913 konkrete Grenzen gezogen wurden. Versuche eigene kurdische Verwaltungen zu etablieren gab es vor allem in den Grenzregionen, in denen Kurden während des 19. Jahrhunderts zwischen Russen und Osmanen taktiert hatten (Günther/Brentjes 2001: 19).

$\mathrm{Zu}$ Beginn des 20. Jahrhunderts war das Ende der Qadscharen-Herrschaft, den Nachfolgern der Safawiden, wie des osmanischen Imperiums absehbar, standen sie bereits unter halbkolonialer Verwaltung. Trotz vielfachen Widerstands teilten Russen und Briten 1907 Persien in Interessenssphären auf (Günther/Brentjes 2001: 24). Unmittelbar nach Landung der Briten am Persischen Golf im Winter 1914 entstand Kontakt zu den Kurden des Welayat Mosul. Der Weltkrieg hatte wegen knapper werdenden Mittel auch Engländer zunehmend auf lokale Kooperation angewiesen sein lassen. Nach der Okkupation der Stadt Kirkuk im Mai 1918 durch britische Streitkräfte entschieden die in der Stadt Sulaimaniya versammelten kurdischen Stammesführer des Gebietes, eine provisorische kurdische Regierung zu bilden. Diese Regierung, die unter der Führung Scheich Mahmud Barzinjis, dem Oberhaupt der bekannten Barzinji-Dynastie, stand, war bemüht freundschaftliche Beziehungen zu den britischen Streitkräften aufzunehmen. In einem Brief an die Briten bat der Scheich ,, not to exclude Kurdistan from the list of liberated peoples." (zitiert nach Asadi 2007: 36). Dies dokumentiert unter Anderem, dass spätestens zu diesem Zeitpunkt patriotische Tendenzen und Ideen nationalstaatlicher Organisationsform einflussreiche Kurden in Südkurdistan erreicht hatten. Der infolge des Weltkrieges von US-Präsident Wilson 1918 entworfene 14Punte-Plan zur Schaffung des Völkerbundes gestand den nicht-türkischen Minderheiten, also auch den Kurden, im zerschlagenen Osmanischen Reich autonome Entwicklung zu.

\subsection{Nation-Building des Iraks}

Das Verhältnis zu den Briten wurde im Folgenden zunehmend gespannter. Kurden unter der Führung Scheich Mahmuds hatten sich zugunsten der Briten bereit erklärt, den Türken 
Widerstand zu leisten. Allerdings verbanden sie dies mit der Gründung eines autonomen Staates unter Einbeziehung iranischer Gebiete, in denen sich bereits der berühmte Führer Simko dem Scheich unterstellt hatte. Briten und Amerikaner, die freundschaftlich mit Iran verbunden waren, mussten das kritisch sehen (Asadi 2007: 9f.). Darüber hinaus schloss Kolonel Wilson, der ab 1918 ziviler Kommissar im okkupierten Irak war, die Errichtung eines kurdischen Staates mit Verweis auf die Unterentwicklung und mangelnde Kommunikation unter den Stämmen generell aus. 1919 erklärte sich Scheich Mahmud mit Unterstützung verschiedener Stämme zum Herrscher über Kurdistan und wandte sich offen gegen den britischen Einfluss (Günther/Brentjes 2001: 30). Obgleich das unausgewogene militärische Kräfteverhältnis zu einem schnellen Sieg der Briten und gerichtlichen Verurteilungen kurdischer Anführer führte, blieben viele Regionen aufständisch (Asadi 2007: 43-51).

Im April 1920 teilten die siegreichen europäischen Mächte die Verwaltung der arabischen Provinzen des Osmanischen Reiches untereinander auf und verwirklichten damit das 1916 geheim geschlossene Sykes-Picot-Abkommen. Dieses sah vor, dass Frankreich den Libanon, Syrien und die Provinz Mosul, England das Gebiet von Palästina bis zur persischen Grenze erhalten sollte. Die Briten beanspruchten nach den Erdölfunden aber das ganze Gebiet des heutigen Iraks. Sie fanden daher die Franzosen mit einem Viertel der Aktien der neuen Erdölgesellschaft, der Iraq Petrol Company, ab. „Iraq nannten die Briten das von ihnen beanspruchte Gebiet von Basra bis in die Berge, das entsprechend der Bevölkerungszusammensetzung in die Liwas Suleimanie und Mosul mit den Kurden, Bagdad mit der sunnitischen und Basra mit der schiitischen Bevölkerung gegliedert war. “ (Günther/Brentjes 2001: 28). Der hierzu 1920 geschlossene Vertrag von Sèvres gestand „Groß-Kurdistan“ Autonomie mit Aussicht auf spätere Unabhängigkeit zu. Allerdings machte der militärische Erfolg der Kemalisten mit Ausrufung der türkischen Republik 1923 diesen Vertrag hinfällig. Der Vertrag von Lausanne, in dem die Westmächte diese neuen Entwicklungen akzeptierten, besiegelte die Hinfälligkeit. Der Völkerbund schlug das Welayat Mosul dem Irak zu, der dadurch zwar um Bodenschätze, aber auch großes Konfliktpotential „,bereichert“ wurde. Kurden reagierten hierauf sehr unterschiedlich (Günther/Brentjes 2001: 29). Trotz weiterer Verhandlungen über die Kurdistan-Frage änderte sich am Ergebnis nichts mehr, denn keine gestaltende Partei hatte ein Interesse die kurdischen Ziele zu unterstützen. Die vom britischen Kolonialminister Churchill 1921 in Kairo abgehaltene Konferenz erläuterte die britische Nahost-Politik und band Südkurdistan in den britisch verwalteten Irak ein. Hier entschied sich auch die Errichtung der irakischen Monarchie mit dem hashimitischen Prinzen Faisal, der als Freund der Briten angesehen wurde, als König Iraks (Asadi 2007: 93-103). Die vom Völkerbund 
forcierte Angliederung Südkurdistans an den Irak war versehen mit Auflagen wie dem Schutz der kurdischen Sprache.

„In der Zwischenzeit waren die Führer der Barzani an die Spitze des kurdischen Widerstandes getreten, eine Naqschbandi-Familie, die nordöstlich von Arbil am Zab ihr religiöses Zentrum Barzan, den „, Ort der Auswanderung“, eine Anspielung auf die Flucht Muhammads von Mekka nach Medina, gegründet hatte. Ihnen schlossen sich die Herki, Zibari, Schirwani und Mitglieder anderer Stämme an. Gründer der Ansiedlung war Scheich Muhammad gewesen, der als religiöser und politischer Führer anerkannt wurde. Um 1900 soll seine Anhängerschaft aus 9000 Personen bestanden haben. "(Günther/Brentjes 2001: 30).

Als 1930 das britische Mandat auslief, boykottierten Kurden die Wahl zum irakischen Parlament und demonstrierten für ihre Unabhängigkeit. Es kam zu blutigen Auseinandersetzungen, die sich wochenlang fortsetzten bis sich kurdische Aghas auf die Seite der Regierung stellten. Starker Widerstand ging vor allem von den unter Mulla Mustafa Barzani Befehligten aus. Weitere Kämpfe entfachten im Kontext von Ansiedlungsversuchen von Assyrern durch staatliche Instanzen und dem Ethnozid der Kurden in Ostanatolien im Namen des türkischen Nationalismus (Günther/Brentjes 2001: 30f.). Mit der Gründung der militaristischen PKK 1978, nachdem lange Solidarisierung durch Zerschlagung von Stammesstrukturen unterbunden wurde, geriet dieser Konflikt in das Interesse der Weltöffentlichkeit.

Ohne weitreichende Veränderungen blieb die erneute Annäherung von Briten und Kurden während des Zweiten Weltkrieges (Asadi 2007: 145). Die materielle Not, die während des zweiten Weltkrieges alle traf, erweckte den Eindruck des Machtverlustes der Kolonialherren. Als Kurden sahen, dass mit sowjetischer Unterstützung die Azeris eine eigene Republik, Aserbaidschan, gründeten, nahm die Führung der etwa 10000 Barzanis Verhandlungen mit Russland auf, und rüstete Mitglieder in der iranischen Kleinstadt Mahabad auf. Mahabad rief unmittelbar nach Aserbaidschan im Dezember 1945 die Republik aus. Allerdings führte die zeitgleiche Einigung von Russland und Iran zum Wegfall russischer Unterstützung, sodass sich Mahabad schutzlos gegen Teheran sah. Der neuen zentralen Führungsgestalt, Mulla Mustafa Barzani gelang mit seiner Gefolgschaft 1947 die Rückkehr nach Barzan und von dort die Flucht ins russische Exil (Günther/Brentjes 2001: 35). Für die etwa 10 Millionen im Iran lebenden Kurden, die während des letzten Jahrhunderts einem starken Assimilierungsdruck durch die Pahlavi-Dynastie wie der Islamisierung ausgesetzt waren, blieb das kurze Aufleben der Republik Mahabad im Jahr 1946 eine Momentaufnahme des Widerstands.

Die folgende Zeit des Kalten Krieges war vom Vordringen der USA geprägt, die vordergründig am Zugang zu den Ölreserven interessiert waren. Kurdische Hauptakteure wie Barzani erfuhren wechselnde Unterstützung. Währenddessen etablierte sich das unabhängige irakische Königreich. Proteste gegen den Anschluss der Barzan-Region an Sulaimaniya führten mehrfach zu Verbannungen in das sowjetische Exil. 
„Zwischen der ersten irakischen Regierung (gebildet am 25.Oktober 1920) und der letzten Regierung in der Zeit der Monarchie (gestürzt am 14. Juli 1958) amtierten 23 Regierungschefs in 59 Regierungen. Von den 16 mal verhängten Ausnahmezuständen wurden nur zwei über das ganze Land verhängt; sechs davon allein auf genau definierte kurdische Gebiete begrenzt. Diese Zahlen spiegeln die permanente Instabilität der politischen Lage in einer Zeit, in der die Briten weitgehend an den Entscheidungen der irakischen Politik mitwirkten und sie darüber hinaus nicht selten diktiert haben. " (Asadi 2007: 149).

1958 führte ein Sturz der sogenannten Freien Offiziere unter Qasim, getragen von panarabischem Gedankengut zum Sturz der Monarchie. Die Kurden hatten sich in der Hoffnung, die neue Regierung würde ihnen entgegenkommen, daran beteiligt (Sarbest 2001: 172). Der Umsturz wurde im Irak mehrheitlich positiv bewertet und Qasim, der Barzani Amnestie gewährte, hatte es nun mit einer nicht mehr zu ignorierenden politischen Kraft im Norden zu tun, die zunächst in den neuen staatlichen Vertragswerken Berücksichtigung fanden.

„Mit Art. 3 der provisorischen Verfassung war eigentlich mehr als ein wichtiger Schritt in diese Richtung gemacht, in dem die Kurden mit den Arabern auf eine Stufe gestellt wurden. Doch die inkonsequente Anwendung dieser provisorischen Verfassung führte zu jener labilen Nachrevolutionsordnung, in der die ungelöste Kurdenfrage ein Symptom für die irakische Krise überhaupt darstellte und zugleich den Prozess der Diktaturherrschaft beschleunigte. " (Asadi 2007:184).

Der wiedergekehrte Barzani beschließt 1961 mit seiner Kurdisch Demokratischen Partei den bewaffneten Widerstand, der bis 1963 von der Sowjetunion unterstützt wurde (Salih 2005:21). Schon 1963 gab es einen weiteren Staatsstreich, bei dem kurzfristig Arif mit Hilfe der BaathPartei als Staatspräsident hervorging. Den Kurden wurden breite Zugeständnisse gemacht, es gab eine Waffenruhe nach Verhandlungen mit KDP-Mitglied Talabani. Schon vor dem Verunglücken Arifs 1966 waren die Hoffnungen erneut enttäuscht worden. Der Nachfolger und ältere Bruder Arif II. wirkte zwar erneut auf eine Beilegung des Konfliktes hin. Politische Instabilität, Sabotageakte und interne Verfeindungen auf kurdischer Seite verzögerten diesen Prozess (Bahjat 2001: 173-175). Das alles hatte nicht nur zur Folge, dass die KDP im Sechstage-Krieg Araber unterstützte, sondern auch, dass sie hierfür die Abspaltung nicht kooperationswilliger Mitglieder und die Errichtung einer konkurrierenden Partei, der Patriotischen Union Kurdistan, hinnehmen musste.

Die von außen unterstütze neue Baath-Regierung verfügte innerhalb kürzester Zeit über viele Waffen und konnte schon im Mai 1965 etwa 12000 Peschmergas mit zwei Panzerdivisionen und 70000 voll bewaffneten Soldaten begegnen (Günther/Brentjes 2001: 43).

„In Anbetracht der militärischen Herkunft der politisch Verantwortlichen in der Hauptstadt war die kriegerische Konfrontation von vorne herein das in Erwägung gezogene Mittel. Aus diesem Grunde nahm der Krieg erschreckende Ausmaße an, so dass man ohne Zweifel sagen kann, dass die Regierungsseite nicht mehr die Verhältnismäßigkeit der Mittel wahrte. Dadurch eskalierte der Konflikt erheblich. “ (Asadi 2007: 229). 


\subsection{Konfliktsteigerung unter dem zweiten Baath-Regime}

Das zweite Baath-Regime (ab 1968) kämpfte zunächst gegen die KDP und bemühte sich die Spaltung der Kurden zu vertiefen. Salih bezeichnet diese Zeit als eine, in der weder Krieg noch Frieden herrschte. 1970 kam es zu einem Abkommen zwischen Baath-Partei und der KDP Barzanis, das ein autonomes Kurdistan (vgl. Anhang I.b) vorsah. Zwei fehlgeschlagene Mordversuche auf Mustafa Barzani 1971 und 1972 legten jedoch die wahren Interessen des Regimes offen (Salih 2005: 28). Das vom Regime bestimmte Autonomiegebiet umfasste nicht mal 50 Prozent der Kurden im Irak (Sharif 1991: 151). Die Beziehungen von KDP und Baathisten standen eine enorme Belastungsprobe aus mit zahlreichen Wortgefechten und offenem Wahlausgang. Es zeichnete sich keine Einigung ab, denn die Regierungsseite sah ihre Pflicht mit der Verkündung des Autonomiegesetzes als erfüllt an, während sie weitere Forderungen der Kurden zurückwies, vor allem die nach dem Anschluss Kirkuks. Dieses Autonomiemodell wurde auf breiter Basis abgelehnt und die Alternative dazu war lediglich die kriegerische Auseinandersetzung (Asadi 2007: 336).

Im April 1974 begannen die offenen Gewalthandlungen. In Kurdistan wurden über 500 Dörfer bombardiert und unter anderem mit Napalm angegriffen (Salih 2005: 33).

Der kurdische Widerstand begann 1975 zusammenzubrechen und wurde gefolgt von einer langatmigen Phase systematischer Verfolgung und Vertreibung. Ein Jahr nach Verkündung der Autonomie war die Perspektive kurdischer Selbstverwaltung in weite Ferne gerückt. Nach einer erzielten Einigung zwischen Irak und Iran, der zuvor die Kurden militärisch unterstützt hatte, verringerte sich der Einfluss der Grenzbewohner erheblich.

„In dieser verzweifelten Lage, umgeben von zwei Staaten, völlig überrascht von der Teilnahmslosigkeit der USA, die ihn seinem Schicksal überließen, befahl Mulla Mustafa Barzani als letzte Entscheidungsinstanz an der kurdischen Front die Einstellung aller Kampfhandlungen. Der kurdische Aufstand, welcher beinahe vierzehn Jahre lang ununterbrochen gedauert hatte, brach in weniger als vierzehn Tagen zusammen. " (Asadi 2007: 355).

„Anfang 1976 wurden kurdische Familien zusammengetrieben, auf Lastwagen verladen und in den arabischen Südirak gebracht, wo sie in Gruppen von bis zu fünf Familien auf arabische Dörfer verteilt wurden. Die evakuierten kurdischen Dörfer wurden anschließend zerstört oder von irakischen und ägyptischen Arabern besiedelt, die als Arbeitskräfte aus Ägypten geholt wurden. " (Havrest 1998: 178).

Die Machtübernahme Saddam Husseins 1979 übertrug den politischen Entscheidungsprozess wie nie zuvor auf den Willen einer Person, dem Präsidenten. Der von Hussein nach Sturz des Schahregimes geführte Angriff auf Iran lenkte sein militärisches Interesse zunächst weg von Kurdistan, so dass es zu einer Art Atempause kam. Um den Einfluss des fundamentalistischen Irans einzudämmen, waren den Weltmächten viele Mittel Recht. US-Präsident Reagan 
erklärte zunächst im November 1983 Saddam Hussein zu einem gemäßigten Verteidiger westlicher Freiheiten. Diesem „moderaten Verteidiger westlicher Ideale“ wurden diverse Kampfstoffe zugeteilt.

„Aus New York kamen die Blaupausen zur Errichtung seiner ersten Giftgasfabrik und die amerikanische Industrie lieferte ihm mit Genehmigung des Pentagons Bakterienkulturen des Milzbranderregers. Großbritannien half mit den erforderlichen Technologien und Rohstoffen für Nervengase wie VX, während der Irak die Produktstätten für Senfgas und Tabun aus der Bundesrepublik Deutschland bezog. " (Günther/Brentjes 2001: 48).

Obwohl der Einsatz dieser Kampfmittel mehrfach belegt wurde und heute noch Überlebende behandelt werden müssen, konnte sich die UNO nicht $\mathrm{zu}$ einer Definition von Kriegsverbrechen einigen.

„Je länger der Krieg mit Iran andauerte, umso stabiler wurde die Regierung in Bagdad, und zwar durch milliardenschwere Daueraufrüstung und Unterstützung aus vielen Staaten der Welt, die sie als Bollwerk gegen den Expansionsdrang des islamischen Iran betrachteten. Diese Entwicklung versetzte den irakischen Präsidenten in die Lage, zu versuchen, die Kurdenfrage militärisch ein für allemal zu beenden. (...) $\mathrm{Zu}$ einer 'Endlösung' der Kurdenfrage konzentrierte die irakische Armee Anfang des Jahres 1988 ein großes Truppenkontingent auf den kurdischen Norden. " (Asadi 2007: 426).

Die sogenannten Anfal-Offensiven, die die demographische Struktur der Region völlig veränderten. Neben über 1,5 Millionen Umgesiedelten forderten sie an die 200000 Opfer. Allein bei dem Massaker von Halabja 1988 waren etwa 5000 Tote zu beklagen. Zudem wurden die kurdischen Parteien während des achtjährigen Golfkrieges zwischen Iran und Irak gegeneinander ausgespielt und verschiedentlich von den Kriegsführern instrumentalisiert.

\subsection{Von der Flugverbotszone zur kurdischen Autonomieregion}

Zu Veränderungen im Umgang mit dem „Kurden-Problem“ kam es erst durch die veränderte Haltung ausländischer Mächte. Wendepunkt hierfür wurde der Überfall Iraks auf Kuwait im Januar 1991. Auslöser dieses zweiten Golfkrieges mochten die Interessen der Westmächte an einem gesicherten Zugang zu den wichtigen Ölquellen sein. Sowohl Kuwait als auch SaudiArabien erzielten im Gegensatz zum Irak bereits seit Längerem größere Profite aus ihren finanziellen Investitionen in der internationalen Wirtschaft als aus den Ölexporten, was sich auf den Ölpreis auswirkte. Unter Anderem dadurch provoziert marschierte Saddam Hussein im Sommer 1990 in Kuwait ein und wurde infolge eines vermeintlich weiteren Vorrückens seiner kriegsgeschwächten Armee nach Saudi-Arabien als neuer Feind von den USA angegriffen und in kürzester Zeit vernichtend geschlagen (Günther/Brentjes 2001: 51f.).

Nach dieser Niederlage des Baath-Regimes kommt es zu chaotischen Zuständen. Im Nordirak gibt es spontane Volksaufstände. Nachdem die irakischen Schiiten weitestgehend als Opposition im Land zerschlagen worden waren, reagiert das Regime mit einer schweren 
Offensive gegen die übrigen Aufständischen, den Kurden. Obwohl die Peschmerga Widerstand leisten, kommt es im April 1991 im Norden zu einem Flüchtlingsexodus, der etwa 15000 Menschen das Leben kostet. Die Weltöffentlichkeit schaut auf die Region und Parolen wie „Blut für Öl“ dominieren die mediale Berichterstattung. War zuvor ein internationales Eingreifen an dem Argument der nationalen Selbstbestimmung des Iraks gescheitert, wurden nun die Genfer Flüchtlingskonventionen als Rechtsgrundlage in den Mittelpunkt gerückt. Mit der Resolution 688 wurde erst eine Flugverbotszone, dann eine Schutzzone im Nordirak eingerichtet. Dieser Präzedenzfall im Völkerrecht ermöglichte den Flüchtlingen zurückzukehren und humanitäre Hilfe in Anspruch zu nehmen (Emanuelsson 2005: 73f.). Die vom Baath-Regime verhängte Wirtschaftsblockade wie auch die anhaltende Bereitschaft der Türkei gegen kurdische Widerständler vorzugehen erschwerten erste Versuche der kurdischen Selbstorganisierung und Selbstverwaltung (Günther/Brentjes 2001:132).

„Als nach dem Golfkrieg 1991 im Norden des Iraks eine Schutzzone der Kurden gegen Saddam Hussein eingerichtet wurde (in der etwa 60\% der irakischen Kurden lebten), kam es zur Bildung eines (dann zweier) kurdischer Proto-Staaten, die bis 2003 über eigene Regierungen, eigenes Militär, ein eigenes Parlament und eine eigene Währung verfügten und faktisch unabhängig waren, wenn auch die völkerrechtliche Anerkennung fehlte. Diese Entwicklung unterstreicht nachdrücklich das Scheitern des baathistischen Nation-BuildingProjektes, das ja den Gesamtirak zu einem arabischen Nationalstaat zu machen gedachte." (Hippler 2004: 125).

Die erlangte Eigenständigkeit stand lange im Schatten interner Spaltungen und Konflikte. Erst 1998 wurde in Washington zwischen den Führern der Streitparteien, Barzani und Talabani, ein Friedensabkommen erreicht, das eine Normalisierung zuließ (Salih 2005: 167).

„The Kurdish parties started an indigenous peace process which included, amongst other things, a ceasefire agreement and renewed coordination between the two administrations, not least in order to ensure implementation of the UN Oil-for-Food-agreement (UN Resolution 986). By the end of 1996, the UN Oil-for-Food agreement, which allocated an annual share of 13 percent of the UN administered oil sales to the Kurds, came into force. The UN agencies were then able to cooperate directly with the KRG which increased the legitimacy of the Kurdish administration. (...) After 1998 positive socio-economic and political developments were visible in Iraqi Kurdistan; these were foremost due to the Oil-for-Food Agreement. The violence ceased. Villages, agricultural lands, education and health facilities were rebuilt and the Kurdish administration, the media and civil society institutions were slowly recovering. (...) The events of the $11^{\text {th }}$ of September dramatically changed US foreign policy, particularly its Middle East policy. In this context the Kurds became an important element in the subsequent developments leading up to the war against Iraq. The Kurds played an active political and military role before during and after the war." (Emanuelsson 2005: 76f.).

Der US-geführte Krieg schuf eine neue machtpolitische Lage für Irakisch-Kurdistan, das 2003 noch in den strategischen, von Saddam Hussein gezogenen Grenzen bestand und dessen Status als Schutzzone mit Teilnahme an der Coalition of the willing auslief. „Die bisher das Land dominierende Gruppe sunnitischer Araber musste nun fürchten,
entscheidend an Einfluss zu verlieren. Sie war in geringem Maße von der Diktatur 
unterdrückt worden, vor allem aus ihr rekrutierten sich die meisten Kader oder Unterstützer", wohingegen die Bevölkerungsmehrheit der Schiiten unter Hussein besonders $\mathrm{zu}$ leiden hatte. Schiiten konnten ,damit rechnen, eine insgesamt dominierende Rolle einzunehmen, wenn sie den anderen Gruppen gegenüber geschlossen auftraten. Die Ausgangsposition der schiitischen Araber war dadurch gekennzeichnet, dass ihre politischen Organisationen zwar von der Diktatur schwer getroffen und massiv unterdrückt worden waren, deren religiös inspirierte Parteien aber im Exil noch existierten. Diese besaßen daher beim Sturz der Diktatur einen wichtigen politischen Vorsprung: Bei ihrer Rückkehr aus dem Iran konnten sie schnell über eingespielte politische Strukturen, Geld und eigene Milizen verfügen. Demgegenüber war der säkulare Flügel schiitischer Araber eigentlich mit starkem Potential politisch kaum handlungsfähig. (...) Bei der kurdischen Bevölkerung, insbesondere in der kurdischen Autonomiezone, sah die Lage grundlegend anders aus als im Rest des Landes. Hier bestanden weiterhin funktionierende politische Strukturen und eine weitgehend intakte Infrastruktur, die in der Zeit seit 1991 aufgebaut worden war (...). Allerdings besteht in der kurdischen Bevölkerung und in ihren Parteien eine massive Stimmung zugunsten der Unabhängigkeit vom Irak, die aus pragmatischen Gründen allerdings nicht öffentlich gefordert wird. Die kurdische Politik drängt deshalb stark auf eine Föderalisierung des Irak und ihre faktische Autonomie als Mindestbedingungen eines Verbleibens im Irak, die bei einem unbefriedigenden Verlauf dann zur Eigenstaatlichkeit ausgebaut werden könnte. Zugleich bestehen starke Tendenzen, die kurdischen Siedlungsgebiete außerhalb der alten Autonomiezone in den kurdischen Machtbereich zu integrieren. "(Hippler 2004: 127f.).

Das weitreichende Chaos im Irak erfasste offenbar nicht in gleichem Maße die kurdische Autonomieregion, die sich weiterhin unabhängig zu entwickeln scheint. 15 Monate nach der ordentlichen Wahl konnte sich eine Regionalregierung Kurdistans bilden, die im Mai 2006 in Arbil vereidigt wurde. Ministerpräsident wurde Nechirvan Barzani, dessen Stellvertreter ein Mitglied der PUK ist. Das aktuelle Kabinett umfasst 42 Minister, darunter 3 Frauen (Fischer Weltalmanach 2007:242). 


\section{Zu Gruppenorganisationen und -dynamiken}

$\mathrm{Ob}$ und inwieweit im südlichen Lebensraum der Kurden, im heutigen Irakisch-Kurdistan, Nationalismus die treibende politische Ideologie ist, ist nicht zu beantworten. Aber deutlich ist doch, dass die Kenntnis demokratisch definierter Partizipationsmöglichkeiten und die Existenz ethnisch legitimierter Nationalstaaten Kurden im 20. Jahrhundert politisiert haben. Im folgenden Teil der Arbeit werden zunächst Aspekte der sozio-politischen Organisationen in nicht-staatlichem Kontext vorgestellt. Eine Auswahl an Ideologien und Prozessen, die die irakische Gesellschaft des 20. Jahrhunderts geprägt haben, wird der Betrachtung der Integration in nationalstaatliche Strukturen vorangestellt. Dies soll einen breiten Überblick gewährleisten, der mögliche komplexe Entwicklungen und Dynamiken erkennen lässt.

\section{Außerstaatliche sozio-politische Strukturen}

Für die Betrachtung sozio-politischer Strukturen, die einen vor- und außerstaatlichen Charakter haben, stehen nur einige wenige, auf Feldforschung beruhende Quellen zu sozialer Organisation der Kurden des südlichen Verbreitungsgebiets ${ }^{24}$ zur Verfügung, so dass diese Darstellungen als stark verallgemeinert und lückenhaft bewertet werden müssen.

\subsection{Verwandtschaftsbeziehungen}

Verwandtschaftliche Beziehungen entstehen durch Heirat und Deszendenz und sind dabei zumeist an ein klares Reglement gebunden. Bei Kurden erkannte Barth, dass allgemein die nahe familiäre Endogamie präferiert, und dabei die Cousinen-Heirat (der FBD) besonders bevorzugt wird (Barth 1979: 10).

Was Eheschließungen betrifft, so fänden ohne Zustimmung der Braut Verhandlungen zwischen repräsentativen männlichen Mitgliedern der Familien statt und sobald ein Brautpreis ausgehandelt wurde, begannen die Vorbereitungen. Bekannt sei, dass der Brautvater eine Heirat mit einem Neffen (dem Sohn des Bruders) bevorzugt arrangierte, wodurch ihm die größten Einflussmöglichkeiten erhalten blieben. Begünstigt würde dies dadurch, dass für Neffen der üblicherweise zu zahlende Brautpreis in der Regel sehr viel niedriger ist. Die günstigste Heiratsvariante ist der Schwesterntausch, bei dem wegen der Reziprozität kein Brautpreis fällig wird. Polygynie, das Ehelichen mehrerer Frauen, ist möglich, wird jedoch

\footnotetext{
${ }^{24}$ Unter Anderem liegt von Barth, dem Theoretiker des hier verwendeten Ethnizitätsbegriffes, die Skizzierung der Situation, wie er sie zu Beginn der 50er Jahre vorfand, vor. Eine weitere wichtige Quelle ist die detaillierte Analyse von Franz aus den 80er Jahren. Übertragbare Ergebnisse einer Feldstudie in der Provinz Hakkari aus dem türkisch-syrischen Grenzgebiet präsentierte Lale Yalçin-Heckmann im Jahr 1991.
} 
nur selten von wohlhabenden Männern praktiziert und dient meist der sozialen Einbindung verwitweter Frauen von Verwandten (Barth 1979: 26-29).

Die innerfamiliären Beziehungen orientieren sich sehr an Erbmöglichkeiten. Mütter pflegen eine sehr enge Bindung an ihre Söhne, da sie bei Todesfall des Mannes in deren Haushalt wechseln. Töchter unterstehen als Zu-Verheiratende ihrem Vater und vererben ihren nahen männlichen Angehörigen ihren Brautpreis. Brüder sind theoretisch gleichberechtigt und heben sich erst durch ihren de facto-Einfluss von einander ab. Es entwickelt sich ein spezielles Bruderverhältnis durch die üblichen Heiratsoptionen der Kinder (Barth 1979:32).

Die von Barth ermittelten Daten stimmen im Wesentlichen mit denen von Yalçin-Heckmann ${ }^{25}$ aus den 90er Jahren überein. Auch sie stellte fest, dass Cousinenheirat präferiert würde:

„Affinal status relations between wife-givers and wife-takers involve a higher status of the former on the basis of the "act of giving" (...) Direct exchange and FDB marriages underplay the status inequality between wife-givers and wife-takers, and are frequently practised together in order to reinforce the tribal solidarity and equality. Bride price and direct exchange however are not always convertible, nor do bride-price and status always match." (Yalçin-Heckmann 1991: 254).

Verwandtschaft, die verschiedene Lineages miteinander verbindet, baut hierauf:

„Tribal membership is claimed and recognized to pass through patrilineal lines but kinship through matrilateral lines are also important. Matrilateral kin, affinal relations and neighbourhood ties often complement the importance of agnatic lines and provide the networks for economic, political and social cooperation." (Yalçin-Heckmann 1991: 133).

Der eheliche Status ist für das Erwachsensein aus verschiedenen Gründen wichtig. Dazu zählt auch der Begriff der Ehre, der im kurdischen Moralkodex beibehalten wird. Mädchen und Frauen gelten weiterhin als größtes Risiko für die Unversehrtheit der Ehre, obwohl sich ihr Rollenmuster stark gewandelt hat und sie mittlerweile in fast allen Berufen und gesellschaftlichen Sphären anzutreffen sind (Fischer-Tahrir 2003: 27-29).

Insofern sich das Leben der Kurden größtenteils in Dörfern und pastoralen Camps der Bergregion abspielte, kam und kommt den patrilokalen Haushalten eine wichtige Rolle zu.

„, The use of space within and around the house is affected by gender roles, age ranking, and kinship position. Claims or counter-claims to status changes are made through subtle strategies involving seating, eating or visiting-patterns. Households are primarily property holding, consumption units, but patrilocal residence and kinship relations are ideologically crucial to the local conceptualization of a household. (...) The labour organization, consumption and production patterns reflect the hierarchy within the household as well as between households. Attitudes towards work and religious and moral identity indicate a differentiated evaluation of work, merit, generousity, and wealth." (Yalçin-Heckmann 1991:182).

\footnotetext{
${ }^{25}$ Yalçin-Heckmann macht überdies weitreichende Angaben zu den genauen Benennungen und Abstraktionen der Familienterminologie. Da sich diese jedoch nur auf einen konkret von ihr analysierten Stamm in der Südtürkei beziehen und es sich meiner Kenntnis entzieht, ob es sich auf Stämme im Nordirak übertragen ließe, findet dies hier keine weitere Erwähnung.
} 
Rigide achteten die Stämme Endogamiegebote, was die Kastenbildung unterstützte. Die Lineage blieb bei Kurden eine stabile Einheit, die maßgebend auf die politische Ordnung wirkte (Barth 1979: 59). Die Organisation in kleinen endogamen Einheiten in Abwesenheit zentraler Institutionen und Rechtsprechung bestärkte die Idee der kollektiven Verantwortlichkeit. Das begünstigte die (Blut-)Fehde als Instrument gesellschaftlicher Regulierungen, wobei dies nicht nur Tötungen einzelner Menschen, sondern auch kriegerische Handlungen der Gruppe verlangen konnte (Barth 1979: 72ff.).

\subsection{Nomadismus und tribale Strukturen}

Wirtschaftlich bedingt ergeben sich für Kurden bestimmte soziale Bezugsgrößen. Kurden bewohnten überwiegend kleine, entlegene Siedlungen in Regionen mit langen Wintern. Diese Landschaft bietet sich vorwiegend für halbnomadische pastorale Produktion an. Vollnomadische Lebensweise half daneben, beispielsweise in Zeiten turkmenischer Durchdringung, Razzien und später staatlichen Interventionen der Osmanen zu entgehen (Behrendt 1993: 116). Im Kontext der tribal strukturierten Dörfer erklärt Barth ${ }^{26}$, stünden sich Arbeiter und Landpächter hierarchisch gegenüber. Die Landbesitzer, eine dritte soziale Klasse, hielten sich vielmehr in nahegelegenen Städten auf. Wesentlichste wirtschaftliche Einheit sei jedoch der arbeitsteilige Haushalt, wobei der Vater Feldarbeit tätigt und Pferde bewacht. Frauen sorgen nicht nur für den Haushalt; ihnen obliegt die Versorgung der anderen Tiere und in den Erntemonaten haben sie auf den Feldern zu helfen (Barth 1979:25).

Der (politische) Zusammenschluss nomadisierender Familien half, sich gegen die zunehmend siedelnden Bevölkerungsteile zu behaupten. „Die Stämme schlossen sich je nach Notwendigkeit zu Verbänden zusammen oder befanden sich mit anderen Stämmen in Konflikten um Weideland, Wasserstellen oder Wanderwege, wobei die Sprachen nebensächlich waren." (Günther/Brentjes 2001: 21). Die überregionale Formation von Stämmen neben den Dynastien ist keinesfalls starr, noch mit klaren Termini festzuhalten, da sie keinen westlich verständlichen politischen Organisationsmustern folgt. Klane schlossen sich zu Stämmen zusammen und teilten sich wieder auf. Stammeszugehörigkeit verlieh nicht nur Prestige, sondern regelte auch Tributzahlungen (Franz 1986: 177-179). Im Unterschied zu der dynastischen Herrschaft der kurdischen Baban (1650-1850) nahm der Einfluss einzelner lokaler Anführer durch persönliche Bereicherung zu, als sie im Zuge des Inkrafttretens osmanischen Landrechts große Landstriche zu ihrem Eigentum erklärten (Barth 1979: 53).

\footnotetext{
${ }^{26}$ Barth bezeichnet die Kurden zur Zeit seiner Beobachtungen als siedelnde oder Transhumanz betreibende Ackerbauern und Viehzüchter. Ein Großteil der Kurden war bereits urbanisiert (1979: 11).
} 
„Kinship obligations and networks are thought to be meaningful and useful in dealing with administrators, politicians and military personnel. Among the local people who work in the city administration, police forces or as clerks, kinship ties with the villagers mean securing a supply of village produce such as yogurt, cheese, butter or honey, goods which are bought at high prices and low quality in town. For the villagers the same relations mean help and advice with all sorts of bureaucratic problems and sometimes a bed in town. Tribal aghas, on the other hand, seek to form kin/affinal links to local administrators. A tribal agha had a ritual parenthood (kirve) relation with the commander of the commando unit and this was thought to have helped in securing the release of the agha's son from detention. The idiom of kinship thus is a fundamental part of the shared culture between the regional and wider national group." (Yalçin-Heckmann 1991: 39).

Barth macht diese tribale gesellschaftliche Ordnung am Beispiel der Jaf deutlich (vgl. Anhang II.a und b). Der Stamm der Jaf bestand fast ausschließlich aus pastoralen Nomaden und umfasste etwa 500 Familien. Die Bezeichnung Ashirat bezog sich auf die lokale wie auch personale Anbindung an die Jaf, die von einem angesehen Beg oder Pasha angeführt wurden. Eine politische Einheit von Landbesitzern war die Tira. Im realen Leben unterteilte sich die Tira in Zeltgemeinschaften und diese wiederum in Haushalte. Eine Vielzahl von ihnen, der Stamm, nahm einen fiktiven gemeinsamen Ahnen aller Mitglieder an (Barth 1979: 37ff.). Eine wichtige politische Rolle kam dabei dem Raiz zu, dem Anführer einer Zeltgemeinschaft. Der Raiz war der direkte Draht zu den Begs (Barth 1979: 40). Die Macht jener war jedoch abhängig von der individuellen Persönlichkeit und regional begrenzt. Beispielsweise diente eine Eheschließung mit der Tochter eines Raiz vielmehr dem Prestige in der eigenen Gemeinschaft als neuen Gestaltungsoptionen (Barth 1979: 49). Während die lokalen Raiz ihre Rolle der informellen gesellschaftlichen Anerkennung verdankten, beriefen sich die Begs auf ihre royale familiäre Zugehörigkeit. Daraus entwickelte sich, dass im Dorf der Anführer gewählt, die Begs jedoch natürlich anerkannt werden sollten (Barth 1979: 43f.).

Ein anderes Beispiel für tribale Organisationen liefern die Hamawand. Sie waren ursprünglich Hirten, die sich durch Überfälle der Karawanen zwischen Persien und Osmanischem Reich bereicherten. Sie umfassten nur bis zu 1500 Individuen, was einer Tira der Jaf entsprach. Diverse Führer werden hier als Agha bezeichnet. Diesen wurde ihr Amt vererbt oder sie wurden gewählt, sofern kein Sohn zur Verfügung stand (Barth 1979: 45-47). Die Hamawand hatten als Räuber bereits militärische Strukturen ausgebildet und verfügten über gute Rekrutierungsmöglichkeiten, sie waren geschult in Waffenkünsten (Barth 1979: 54ff.).

Obgleich das Kurdische kein Wort für Macht parat hält, scheint doch das politische Denken davon geprägt zu sein, dass der politische Einfluss sich proportional zu dem Status der Person verhält. Auf diese Weise wird der politische Einfluss auch anders konzeptualisiert, da der Status weit mehr als die offizielle und formelle Funktion bedeuten kann. 
"A high status position in these hierarchies affords the holder the right to apply certain sanctions, positive or negative, to persons inferior to him in the hierarchy. He is thus able to influence the actions of his fellow villagers in much the same way as a political office holder; He has power." (Barth 1979:78).

Die Herrscher der Stämme von Soran, Badinan und Baban $^{27}$ konnten zwar die Zentralisierungsversuche auf später irakischem Gebiet bis Mitte des 19. Jahrhunderts überstehen und die feudalen Strukturen sich allgemein trotz aller Veränderungen nach dem 2. Weltkrieg erhalten, die Fixierung der Staatsgrenzen hat aber vielfach zur Verarmung der Nomaden geführt (Edmonds 1971:88). Die formale Integration der Gebiete in Nationalstaaten machte die Region zudem anfällig für den Schwarzmarkthandel und entsprechend anfällig für den Verlust früherer Autoritäten. „Some of the old ruling families have disappeared altogether from the political scene but having a tribal base and keeping kinship networks working are still important assets in political competition." (Yalçin-Heckmann 1991: 125).

\subsection{Junge und alte „Dynastien“"}

„Die sozialanthropologischen Begriffe wie „Stamm“, „,Klan“ und „Lineage“ lassen sich nicht ohne Weiteres auf die Realität der kurdischen Gesellschaft übertragen. " (Özdemir 2006:24). Verschiedene Autoren listen daher Stämme, beziehungsweise Dynastien, entsprechend unterschiedlicher Kriterien auf. Umso schwieriger wird die Beschreibung, da sich tribale Strukturen in den letzten beiden Jahrhunderten sehr veränderten.

Die Stadt Sulaimaniya ist hierfür ein Beispiel. Während der osmanischen Herrschaft wurde sie relativ unabhängig von der Baban-Familie regiert. Diese berief sich auf ihre royale Lineage und klassifizierte „Stammeszugehörige“ vor allem über den Status (Barth 1979: 60). Die Verwaltung dieses urbanen Zentrums hatte bereits viele der vorigen tribalen Organisationsformen zerschlagen. Nachdem eine Intrige 1851 die Baban-Herrschaft beendete, nahm der Einfluss des Jaf-Stammes in der instabilen Region zu. Die Jaf konnten ihre Macht durch den Einbezug der Landbesitzer mehr denn der Stammesführer konsolidieren, solange bis sich die Barzanis um die Wiedererrichtung des Modells der Baban in Sulaimaniya bemühten (Barth 1979: 14).

$\mathrm{Zu}$ den wichtigen Stämmen des Irak zählen gemeinhin: die Badinan als Lokaldynastie um Amadiya, die Barzani, seit sie vor etwa 100 Jahren tribale Strukturen hervorbrachten. Weiterhin die Dhauda um Kirkuk sowie die Dizeyi und Lolan in und um Arbil, Hamawand, Jaf, Mamesh, Pira, Pishdar und Zangana (Franz 1986: 185-196). Die Unklarheit bei der Ausweisung und Benennung von Stämmen geht vor allem auf einen wichtigen Wechsel in der

\footnotetext{
${ }^{27}$ Diese gehörten bereits zu den vom Sharafname im 16. Jahrhundert gezählten Fürstentümern (Franz1986:37).
} 
Führungsideologie zu Beginn des 20. Jahrhunderts zurück. Sayyids ${ }^{28}$ und Scheichs profitierten zunehmend von pan-islamischer Ideologie und der Protektion osmanischer Sultane. Aufgrund ihrer religiösen Machtbasis konnten sie auch überregional Kräfte bündeln und ihren gesellschaftlichen Einfluss vergrößern (Yalçin-Heckmann 1991: 123). Anhänger der weit verbreiteten Derwisch-Orden waren in der Regel Bauern, deren Heilserwartungen sich auf die Nähe zu einem Scheich richteten. Über die Zugehörigkeit zu einem Derwischorden sagt Barth: „Membership in a brotherhood offers subjective satisfaction, giving the underprivileged person a field in which he feels that he too can manipulate and control power, seems the only constructive alternative." (Barth 1979: 89). Die emotionale Gebundenheit an die Derwische, die Scheichs, gründete sich auf individuelle spirituelle Erfahrungen während der Rituale, die sich sehr von den Praktiken des orthodoxen Islam unterschieden. Der Derwisch verfügte im Gegensatz zum Mullah über keine klar definierte Rolle, übernahm aber in Gegenden in denen ein Mullah fehlte oft dessen Aufgaben. Scheichs und Sayyids erlangten nicht selten materiellen Wohlstand durch die Schenkungen, die ihnen zur Bestätigung ihres sozialen Status gemacht wurden. Die beiden Sufi-Orden der Naqschbandiya und Qadiriya akkumulierten Prestige und Einfluss in den eigenen Reihen. Ihnen oblag die religiöse Führung, sofern es noch alte Herrscherhäuser gab. Darüber hinaus übernahmen sie juristische Funktionen als Schlichter (Behrendt 1993: 177-181). Die ScheichFamilien neigten zu engen endogamen Familienverbänden, wobei das Streben nach höheren sozialen Positionen eheliche Vermischung mit lokalen Dynastien häufig werden ließ (Barth 1979: 90). Denn für den Scheich ergaben sich Prestige und Status nicht nur aufgrund seiner theologischen Kenntnisse durch das Studium des Korans, sondern auch aus seiner lokalen Verortung (Barth 1979: 93).

Die Übernahme ziviler Aufgaben infolge des einhergehenden Wandels der Rolle von ScheichDynastien und den unter dem Druck der osmanischen Zentralregierung an Einfluss verlierenden Stammesführern eröffnete charismatischen Personen neue Einflussmöglichkeiten. Scheich Ubaydullah Nehri, Oberhaupt der Naqschbandiya, markierte vorerst den Höhepunkt der neuen Gesellschaftsschicht. Viele Autoren bezeichnen den von ihm 1880 geführten Aufstand gegen die Osmanen als Beginn der modernen kurdischen Nationalbewegung (Behrendt 1993: 115-220).

Die regional begrenztere Qadiriya unterteilt sich in zwei Lineages: die Barzinji, die sich auf das Zentrum Sulaimaniya konzentrierten, und die Talabani um Kirkuk. Die Barzinji, deren

\footnotetext{
${ }^{28}$ Sayyid ist ein arabischer Ehrentitel für diejenigen, die sich auf eine Abkunft aus der Familie des Propheten Muhammads stützen können. Oft wird damit auch das legitime Tragen verschiedener Symbole, wie dem Turban verbunden. Sie sind vielfach anerkannte Theologen.
} 
markanteste Figur der bereits erwähnte Scheich Mahmud bleibt, berufen sich auf die Abkunft eines Sayyid Isa, der im 15. Jahrhundert in Kurdistan siedelte. Die Talabani-Scheichs stützen sich auf keine prophetische, sondern eine unklare Abkunft aus der Eheschließung eines aus Indien stammenden Scheichs mit der Tochter eines Stammesführers der Zangana, so dass sie mit jenen verbündet und regional auf deren Erbe in Kirkuk und der Provinz Arbil angewiesen waren. Schon im 19. Jahrhundert entwickelte sich zwischen den beiden Familien eine teils gewaltsam ausgetragene Rivalität. Die Barzinji-Scheichs „rekrutierten“ vor allem unter den urbanen Kurden und erlangten so Einfluss im ganzen irakischen Gebiet. Zu ihrer Gefolgschaft gehören Stammesmitglieder der Mamesh und die Region Sulaimaniya (van Bruinessen 2000: 213-227). Freundschaftlich waren auch die Beziehungen zu den Barzanis, einer wichtigen Scheich-Dynastie der Naqschbandiya, deren Ursprung nicht deutlich ist, aber auf einen Taj ad-Din, der Mitte des 19. Jahrhunderts in Barzan einige Bauern für sein Tun gewinnen konnte, zurückgeführt wird. Neben deren berühmten politischen Mitgliedern wie Mustafa Mullah, wirkten und wirken Einige als Vertreter des islamischen Extremismus (1986: 161172).

Die Verquickung von tribalen, religiös determinierten Führungsschichten und lokalen, familiären Bindungen, die es so schwer macht, die gesellschaftliche Struktur zu erfassen, muss bei der Betrachtung von Machtoptionen den Blick auf die zugewiesenen Status lenken. Eine von Küchler 1978 entworfene Skizze zeigt die verschiedenen prestigeträchtigen Rollen der kurdischen Gesellschaft gegen Mitte des 20. Jahrhunderts (vgl. Anhang IX).

Das von Yalçin-Heckmann untersuchte Beispiel der Hakkari macht abschließend deutlich: „Ethnic identity as the non-corresponding kinship and tribal structures present a very flexible picture of loyalty and identity. It provides for flexibility in interpretation and actions, it leads to difficulties and misunderstandings as well. A local military officer who accepts gifts and visits from a group of villagers on a regular basis may be thought to be forming kinlike ties with these people but at a critical point the acceptance or refusal can be interpreted as a pro- or anti-Kurd attitude.(...) The crux of the matter is, however, the rigideness of the political system in accepting ethnic structures and identity as something different from localism, regionalism or tribalism. The common idiom of kinship provides for important channels of communication and exchange between the local and national cultures. However, the unmistakable mixture of ethnicity together with kinship and tribe makes the local political phenomena distinct." (1991: 40).

\section{Identitätsstiftende Momente für die Bevölkerung Iraks}

Insofern sich für Kurden der Bedarf nach einem eigenen Nationalgefühl als Reaktion auf den Assimilierungsdruck entwickelte, werden nun einige der Aspekte nachgezeichnet, die diesen Druck und eventuell das reaktionäre Betonen ethnischer Marker provozierten. 
Denn Kurden im heutigen Nordirak erlebten viele weitreichende gesellschaftliche Umwälzungen.

Die Auswahl der identitätsformenden Aspekte orientiert sich an folgender Überlegung: "According to van Bruinessen, in all three countries some Kurds have assimilated, yet many who politically identify themselves as citizens of their state have kept or developed their kurdish identity. As a matter of fact, many vacillate between the latter orientation and 'a more nationalistic one'. In due course, the secular and popular conception of the Kurdish national identity has replaced the earlier aristocratic, religious and/or tribal one, thereby creating new bases for Kurdish nationalism. Influencing factors were modernisation, socioeconomic changes, as well as European nationalism, Kemalism, pan-Arabism and the struggle of the Kurdish national movements but also migration and exile. (...) The Kurds in Iraqi Kurdistan carry a cluster of possible social identities or tendencies which they can and will develop depending on the situation." (Emanuelsson 2005: 71).

\subsection{Irakisierung, Panarabismus und die Arabisierung Iraks}

„, The new state of Iraq was a British construction and its nation-state building differed from Turkey and Iran in terms of external interferences, institutional building and legitimacy. It has not been entirely possible for the regimes in Baghdad to systematically exclude Kurdish representatives and the Kurds have, at least in principle, secured the right to education, publishing in Kurdish as well as regional autonomy." (Emanuelsson 2005: 67).

Für den Irak galt es, im Gegensatz zu anderen Ländern in denen Kurden als Minderheiten lebten, eine nationale Identität neu zu erfinden. Als die Briten sich auf eine Form der indirect rule stützen wollten, setzen sie Faisal, der sich zuvor von der arabischen Nationalbewegung in Damaskus zum König hatte ausrufen lassen, für diesen Zweck ein. Eine vom britischen Kolonialverwalter Leachman seinerzeit betriebene Umfrage im Welayat Mosul belegte ethno-politisch eine sehr anti-arabische Haltung. Es folgte die Empfehlung einer eigenen kurdischen Staatsgründung, die jedoch von den Entscheidungsträgern ungehört blieb (Asadi 2007: 43). Eine weitere Umfrage anlässlich der Kairoer Konferenz kam zu dem Ergebnis, dass sich zwar die kurdischen Gebiete mehrheitlich für das britische Mandat aussprachen, jedoch sowohl den Anschluss an den Irak wie auch eine arabische Regierung ablehnten. Diese Haltung schien sich mehr auf das Misstrauen gegenüber Arabern zu begründen, denn auf kurdisches Nationalgefühl. Das erschütterte die Briten in ihrem Glauben an einen leichten Zusammenschluss (Asadi 2007: 96).

Dass der Irak auch in den folgenden Jahren von britischen Interessen geleitet blieb, verhinderte das Ausbreiten nationalistischer Tendenzen wie in der Türkei (Asadi 2007:147). An dieser Stelle sei schon darauf hingewiesen, dass gegen Ende des ersten Weltkriegs auch Propaganda pro-türkischer Kreise Bilder von Briten als grausame Christen entwarf, um das Gemeinschaftsgefühl der islamischen Mehrheiten zu stärken (Asadi 2007: 53).

Unter monarchischer Führung blieben die Auswirkungen auf die kurdischen feudalen Strukturen überschaubar, denn die Aghas behielten ihren Status quo als Großgrundbesitzer. 
Zeitgleich begann jedoch der Panarabismus, der die Wiederbelebung der arabischen Nation forderte, eine starke Anziehungskraft auf junge Iraker auszuüben (Strohmann 2003: 123). Arabischer Nationalismus war im Wesentlichen eine Reaktion auf das Osmanische Reich. „Arab nationalism arose out of the failure of its immediate predecessor and its ideological parent, Islamic modernist Ottomanism. The movement made progress before 1914, but it remained a minority movement until 1918, when the Arab revolt, the British agreement with the Amyr Husayn, and the British defeat of the Ottomans left the dominant faction of the Syrian and Iraqi Arab notables with no alternative to Arabism." (Dawn 1991: 23).

Die panarabische Bewegung im Irak bestand zunächst hauptsächlich aus einem Bund von Offizieren, die sich Ende der 1930er Jahre gegen die Staatsgewalt und britische Besatzung auflehnten. Sie wurde massiv verfolgt und zerfiel als aktive Bewegung. Ihre Anhänger trugen ihre nationalistische Ideologie in die Baath-Partei hinein und beflügelten dort die spätere Arabisierungspolitik mit entsprechender Wirkung für die Kurden (Sharif 1991: 41).

"In 1958, when the pro-Western Monarch of Iraq was overthrown in a military coup, Barzani was invited back by the new rulers who were willing to recognize the Arabs and the Kurds are two distinct national groups within the Iraqi State and to promise the Kurds extensive autonomy. However, due to conflicts between left-wing pan-Arabists and left-wing Arab (Iraqi) nationalists a number of successive military coups prepared the ground for the take over by the former group (the Baath party) in 1968. Gradually, the Baath party switched to an extreme form of Arab nationalism inseparable from the party, built a repressive superstructure and liquidated most of the internal opposition." (Emanuelsson 2005: 68).

Das Misstrauen gegenüber arabischen Eliten seitens der Kurden wuchs mit dem Verbot der KDP durch Offizier Qassim, nach dessen Sturz sich eine Koalition von arabischnationalistischen Kräften: den Baathisten, Nasseristen und Nationalisten formierte (Asadi 2007: 188). Der Panarabismus der Regierung drückte sich unter Arif I. in der Annäherung zu Ägypten aus. Zum Einen erhielt die schwache, unpopuläre Regierung Rückendeckung von Ägypten, zum Anderen konnte man so die arabische Welt gegen die Kurden mobilisieren. Je mehr der panarabische Leitgedanke im Irak vordrang, desto misstrauischer wurden die Kurden und widersetzten sich (Asadi 2007: 474). Die darüber hinausgehende Orientierung an den arabischen Emiraten bestärkte den kollektiven arabischen Widerstand gegen Israel und stellte den Panarabismus in den Dienst anti-israelischer Parolen. Kurden wurden nun allzu gerne als "zionistische" Agenten bezeichnet. Obwohl es einige kurdische Juden gibt, die Bezug zu ihrer Heimat pflegen, haben Kurden wenige Berührungspunkte mit dem arabischisraelischen Konflikt (Naamani 1966: 292).

Panarabismus, verstanden als ,a near-sacred belief, transnational in character, emphasizing the unitary nature of the Arab World" (Fuller 1993: 118), fand einen wichtigen Vertreter in Saddam Hussein. Er ließ ihn seine machtpolitischen Strategien offiziell leiten, doch führte er tatsächlich eine radikale Arabisierungspolitik des Iraks. Die ölreichen Regionen Kirkuk, 
Sinjar und Khanqin wurden von ihm mit Arabern zwangsbesiedelt, während Kurden vertrieben wurden. Während der späteren Anfal-Offensiven wurden Bäume verbrannt und Brunnen vergiftet oder zugeschüttet, um die Rückkehr der Bewohner zu verhindern. Zwischen 1960 und 1990 wurden etwa 4000 Dörfer gänzlich zerstört (Bruinessen 1999: 4). Zur Arabisierungspolitik gehörte auch das Umdefinieren von Distriktgrenzen in den 70er Jahren; umschloss beispielsweise der Distrikt Kirkuk 1976 fast ausschließlich Kurden, relativierte die willkürliche Grenzveränderung deren Mehrheit, was heute noch den Anschluss der Region an Kurdistan erschwert (Asadi 2007: 503). Wie folgenreich Husseins Politik der Fragmentierung war, zeigte sich, als 2004 der Anführer der KDP, Masud Barzani, nicht nur eine Erweiterung des Gebietes, sondern auch eine Ausweisung aller angesiedelten Araber forderte. Nach Berichten des amerikanischen Kongresses kehrten seit Kriegsbeginn nicht nur 30000 Kurden zurück nach Kurdistan, sondern es zogen auch 100000 Araber weg. Besonders brisant bleibt die Lage in Kirkuk, wo Kurden und Araber um Gebietsansprüche streiten (vgl. http://acd.iiss.org). Andererseits ist das Interesse führender Kurden groß, die Zahl der eigenen Gruppe möglichst hoch anzusetzen und gerne werden andere ethnische Minderheiten der Peripherien ohne Nachfragen den Kurden zugerechnet (O’Shea 2003: 43).

Allgemein muss berücksichtigt werden, dass die militärische, traumatische Niederlage von 1967 die auf arabischem Nationalismus begründete Ideologie entmystifizierte. Es wurden neue ideologische Bezugsrahmen gestärkt, in denen die irakische Nationalisierung sich abspielen konnte. „Dieser Wandel erfolgte als Wechsel von der säkularen zur islamischen Ideologie." (Meier 1995: 54). Nach dem Sturz des Baath-Regimes belegten Wahlen im Dezember 2005 diese Ablösung durch rigorose Islamisierung der Politik (Asadi 2007: 498). Nachdem Kurden an der Seite der Amerikaner gekämpft hatten, nahmen die Feindseligkeiten gerade sunnitischer Araber, die ihnen diese Kooperation vorwerfen, zu (Bengio 2005: 183).

\subsection{Sozialismus und Kalter Krieg}

Neben dem (Pan-)Arabismus beeinflussten der Sozialismus und die politischen Konstellationen im Kalten Krieg die verschiedenen Akteure auf irakischem Boden. Sozialismus als Ausdruck liberaler Traditionen und Inkonsequenzen im Gleichheitsgrundsatz ist ein europäisches Konstrukt. Der organisierte Sozialismus in Form von Parteien entstand in Mitteleuropa parallel zum Nationalismus. So berief sich auch Stalin auf den Nationalstaat als Bezugsgröße des sozialistischen Gedankens als einem bestimmten Wirtschaftstypus (Reiterer 1998: 255). Der Sozialismus arabischer Nationalisten sollte dabei vor allem beim Verwirklichen einer nicht-feudalen und post-kolonialen Gesellschaft helfen. Neben dem ägyptischen Nasserismus vertrat dies der syrisch-irakische Baathismus. Die Gründung der 
Baath-Partei 1941 in Damaskus ging, ohne auf den Islam Bezug zu nehmen, mit der Forderung nach der Vereinigung aller arabischen Länder einher, um sich gegen westliche Hegemonie zu behaupten. Der Rückgriff auf Sozialismus scheint dabei willkürlich und mit der tatsächlichen Sakralisierung der jungen Staatssysteme kaum vereinbar (Meier 1995: 62). Aber nicht nur verwerteten Iraker sozialistische Ideologie, sondern der konfliktreiche Irak hatte einen eigenen Platz im Ost-West-Konflikt eingenommen. Dazu zählten insbesondere der überregionale Charakter des Kurden-Konfliktes und die Beziehung Bagdads zu der Sowjetunion. Die mit den anti-kommunistischen Staaten Türkei und Iran verbündeten USA neigten eher zu einer ablehnenden Haltung gegenüber den Kurden. Dagegen war seit 1958 der Umgang mit dem Irak und den Kurden seitens der Sowjetunion besonders freundlich. Das entspannte Verhältnis zur Türkei verhinderte jedoch ein deutliches Bekenntnis zu den Kurden im Nordirak. „,Darüber hinaus wies die Struktur der Sowjetunion selbst unzählige ethnische und anti-nationalistische Bruchstellen auf und präsentierte, wie sich später herausstellte, kein Musterbeispiel für die Lösung solcher Problematiken. “(Asadi 2007: 274).

Der Kalte Krieg motivierte im Falle des politisch instabilen Iraks die Blockstaaten zum wechselseitigen Unterstützen der Putschisten oder Widerständler (vgl. Anhang VII). Trotz der sozialistischen Außenansicht des baathistischen Iraks war die Anbindung zu den Westmächten eng. Als im Januar 1967 der Krieg im Irak ausbrach, unterstützten Sowjets die Kurden und gewährten ihnen Exil, während die irakische Armee reichlich Waffen aus dem Gebiet der Nato erhielt. Auch deswegen war die Kommunistische Partei im Irak (gegr. 1934) ein wichtiger Partner bürgerlicher Kurden (Strohmann 2003: 123). Auch kommunistische Ideologie hatte Zugang zu der urbanen, sich neu entwickelnden Bildungsschicht gefunden. „Neben die Notabeln und Aghas der Stämme und Städte traten seit den dreißiger Jahren
politische Bewegungen auf demokratischer Basis. Sie entwickelten sich unter der jungen
kurdischen Intelligenz im Irak. Im Vergleich zum Iran und zur Türkei hatten die Briten in
ihrem Mandatsgebiet einen liberalen Kurs eingeschlagen und zumindest im Norden des
Landes ein kurdisches Volksschulwesen zugelassen. Dies ebnete einer kleinen Anzahl junger
Angehöriger der kurdischen Oberschichten den Weg an die neue Universität in Bagdad und
damit zu den Idealen der Demokratie und bürgerlichen Freiheiten. Zudem trafen sie auf die
sich entfaltende arabisch-irakische Nationalbewegung, die Beziehungen zu der Linken in
Großbritannien unterhielt." (Günther/Brentjes 2001:38). Der Zusammenbruch der Sowjetunion hatte erschwerende Auswirkungen auf den Irak. Das Baath-Regime, das zuvor den Ost-West-Konflikt für die Nationalisierung der eigenen Ölproduktion nutzen konnte, unterhielt hauptsächlich Wirtschaftsbeziehungen zum Westen, der daran interessiert war, ihn gegen den Iran und den Osten zu rüsten. 1990 schwand jedoch das Interesse der Westmächte an dieser Armee. Nunmehr lag der Fokus in der Sicherung der Ölquellen, ohne auf die humanitäre Situation im Land zu schauen (Sharif 1991: 157-161). 


\subsection{Politisierung (islamischer) Glaubensgemeinschaften}

Die Politisierung von islamischen Glaubensgemeinschaften wirkte sich vielfach auf den Alltag von Kurden aus. Zum Einen erlebten sie den sozio-politischen Aufstieg der eigenen Sufi-Bruderschaften, Panislamismus, der dem Panarabismus folgte, und nicht zuletzt die schiitische Revolution im Iran. Was die Islamisierung allgemein betrifft, so mögen die nomadisierenden Kurden wohl weitaus geringer von den zentralistischen Bemühungen des Kalifats betroffen gewesen sein, jedoch wurden auch sie früh „,bekehrt“, als Bagdad im 9. Jahrhundert sunnitisches Zentrum wurde. In den politischen Wirrungen rund um den russischtürkischen Konflikt des 19. Jahrhunderts solidarisierten sich einige Kurden mit Osmanen auf der Basis des islamischen Glaubens und empfanden dies als entsprechendes Politikum.

„Die Kluft zwischen den christlichen Völkern der Region, den Armeniern und Assyrern, und den muslimischen Kurden und Türken wurde immer tiefer. Die Prinzipien des islamischen Rechts, die in den vorhergehenden Zeiten das Miteinander zwischen ihnen regelten, verwandelten sich in die religiöse Maskierung eines immer blutiger werdenden Chauvinismus nach dem Vorbild europäischer Bewegungen. " (Günther/Brentjes 2001: 19).

Die regionale Einmischung der Großmächte verschärfte religiöse Gegensätze. Die Niederlage der Türken und der deutschen Verbündeten hatten 1918 zum Ende des Sultanats geführt. „Die neue, relativ starke Autorität in Anatolien verbreitete mit Erfolg auch in Südkurdistan eine wirkungsvolle, anti-britische Propaganda für die Rettung des Islamischen Reiches. Diese Propaganda war stark mit religiösen Parolen besetzt. “(Asadi 2007: 99).

Von neuer politischer Relevanz wurde der sunnitische Charakter der kurdischen Muslime, als König Faisal auf sie verwies, um die sunnitische Mehrheit im Land sicherzuwissen.

Die bereits beschriebene Genese dynastischer Orden politischer Scheichs war noch wesentlicher. Während sich diese religiösen Führer noch zur Zeit der Baban-Herrschaft politisch zurückhielten, bemühten sie sich nach deren Zusammenbruch, das entstehende Machtvakuum zu füllen. Als Scheich Mahmud als König von Kurdistan auftrat, stützte er sich nicht auf die traditionellen kurdischen Lineages, sondern den Islam (Barth 1979: 64f.). Die von den Orden verbreiteten Religionsauffassungen stimmen oftmals nicht mit dem orthodoxen Islam überein, denn Scheichs sind stärker der Sufitradition als der Sunna verpflichtet. Doch bot die theologische Lehre nun eine neue Zugangsmöglichkeit zu politischer Macht, die es zuvor derart nicht gegeben hatte.

„Durch den Aufstieg der Sheikhs in die Führungsspitze der gesellschaftlichen Hierarchie erhielt das religiöse Moment zwangsläufig eine gesteigerte Bedeutung im politischen Geschehen. Man könnte diesen Sachverhalt aber auch ebenso gut umgekehrt formulieren, dass nämlich die rasante Zunahme religiöser Konflikte - hauptsächlich längs der Trennlinie Muslim/Christen - innerhalb der kurdischen Gesellschaft im letzten Viertel des 19. Jahrhunderts das ideale Umfeld für politische ambitionierte Sheikhs darstellte, weshalb sie selbst frühzeitig an der Gestaltung dieser Konfrontation mitwirkten. “ (Behrendt 1993: 226). 
Ein weiteres wichtiges Moment gesellschaftlicher Islamisierung war der Sturz des Schahs und die sukzessive Konsolidierung der Macht der schiitischen Kleriker im Iran, die Asadi als „erdbebenartige Entwicklungen, die den Gang der Ereignisse im Irak enorm beeinträchtigten. “ bezeichnet (2007: 424). Im Iran hatte die Marginalisierung der Kurden sich unter dem Schahregime noch durch ausbleibende Demokratisierung oder Zugang zum Bildungswesen ausgedrückt. Nach Ausrufung der Islamischen Republik wurde der panislamische Gedanke Grund für das Ausblenden kurdischer Interessen, denn Sprachunterschiede höher zu bewerten als die islamische Einheit galt nun als staatsfeindlich. 1980 waren nach Androhung des Heiligen Kriegs alle kurdischen Gebiete des Irans wieder unter Kontrolle des Staats, was die Perspektive auf ein Groß-Kurdistan schwächte.

Aber nicht nur das iranische Regime nutzte den Islam für die Zentralisierungsversuche. Auch das Baath-Regime wusste geschickt pan-arabische und pan-islamische Ideologie im Kampf gegen die Kurden zu verknüpfen. ${ }^{29}$

„For the Baathist architects of the Anfal operation, the Kurds became the unbelievers - those who insisted on remaining outside the faith, embodied now not in Islam but in repressive Baathist state and its fervid brand of Pan-Arabism. "They shall be punished for their unbelief", it is written in the eighth sura. And so with the commencing of the Anfal operation, it came to be. Clever Baathist ideoloques had done their homework when they chose their code name. They knew that in the Middle East the past is rarely over and done with; it is often used to weigh heavily on life in the present." (Makiya 1992: 57).

Bei Betrachtung der nationalistischen Rhetorik sollte nicht ausgeblendet werden, dass die Erfindung von (und der Rückgriff auf) Traditionen von allen Seiten benutzt wird. Geschichte findet sehr selektiv Verwendung, um beispielsweise das Bild der eigenen Unterdrückung zu stärken ,,Yet, for example, Yezidi Kurdish oral traditions recount in considerable detail their past massacres by Muslims. In turn it must be added that the sixteenth century Yezidi Emir of the Soran principality reputedly used his position to persecute local Shiite Muslims. "(O'Shea 2003: 35). Es scheint zudem, dass die neuerliche Hervorhebung der Yeziden als kurdischer Marker sich nicht nur darin gründet, dass sich diese als Ur-Kurden bezeichnen, sondern vor allem in dem wachsenden Bedürfnis, sich zum weltweit kritischer bewerteten Islam abzugrenzen (Franz 1986: 110). Seit Beginn des 21. Jahrhunderts mehrten sich auch die Übergriffe auf kurdische Instanzen durch islamische Fundamentalisten, denen unterstellt wird, das islamische Recht in den von ihnen kontrollierten Gebieten einzuführen und enge Kontakte zu al-Qaida zu unterhalten. Besonders Kämpfer der Gruppe Ansar al-Islam, kurdische Extremisten die sich in den Bergregionen niederließen, griffen an und töteten 2003 gar den Sicherheitsbeauftragten der PUK, Hama Hussein (US-Congress 2002/03).

\footnotetext{
${ }^{29}$ Ein Beispiel hierfür ist bereits die Bezeichnung der militärischen Vernichtungszüge als al-Anfal, was in Bezug auf religiöse Bekenntnisse so viel bedeutet wie "mehr als die Pflicht tun!"
} 


\subsection{Modernisierung der Infrastruktur}

Inwieweit man Aussagen wie „,Some Kurds are as primitive today as their ancestors were at the dawn of civilization." (Naamani 1966: 280) zustimmen konnte oder kann, bleibt dahingestellt, aber dass die geographischen Bedingungen den Ausbau moderner Infrastruktur und Kommunikation verzögerten, ist gewiss richtig.

Nachdem das Osmanische Reich zu Beginn des 19. Jahrhunderts zur Sicherung des stehenden Heeres kurdische Stämme erneut unterworfen hatte, folgten staatliche Reformen, die vor allem die Ökonomie in den kapitalistischen Weltmarkt einbinden sollten. Die Umstrukturierungen betrafen traditionelle Handwerkszweige, die von europäischer Massenproduktion verdrängt wurden. Die irakischen Provinzen gerieten ins Blickfeld der Europäer, als 1861 die dampfbetriebene Schifffahrt auf Euphrat und Tigris eingerichtet wurde. „Doch nach Kurdistan schließlich drangen die durch die Gesetzmäßigkeiten des Weltmarktes diktierten neuen ökonomischen Beziehungen selbst bis zur Jahrhundertwende nicht in nennenswertem Maße vor. " (Behrendt 1993: 159). Mit Öffnung des Suezkanals verlor Kurdistan als Knotenpunkt der Handelsrouten sogar noch an Bedeutung. Selbstständig konnten kurdische Exilanten allerdings seit 1889 mit der Zeitung Kurdistan, in Kairo, Genf und London publiziert, in gewissem Maße eine kurdische Öffentlichkeit schaffen. Sie erschien auf Kurmanji und Osmanisch und veröffentlichte kurdische Epen, stellte aber 1902 schon wieder die Auflage ein (Ağuiçenoğlu 1997: 201).

Das Eindringen zentralstaatlicher Autorität führte also nicht unmittelbar zu höherem Integrationsniveau der Kurden oder nationalem Bewusstein.
„Weder eine Zunahme der Verkehrs- oder Kommunikationsmöglichkeiten, noch eine Steigerung der sozialen Mobilität aufgrund eines etwaigen Zerfalls traditioneller Lebensformen, noch ein Eindringen marktorientierter Warenproduktion, noch eine Herausbildung eines aufsteigenden Bürgertums spielten in der kurdischen Gesellschaft in den ersten Jahrzehnten dieses Jahrhunderts eine wichtige Rolle. Eisenbahn und ausgebaute Straßen erreichten Kurdistan erst lange nach dem Ersten Weltkrieg, und die Anbindung an das Osmanische Post- und Telegrafennetz hatte (...) hauptsächlich Auswirkungen auf die interne Hierarchie des Staatsapparats, eine gesamtgesellschaftliche Wirkung blieb aus." (Behrendt 1993: 391).

Kurden blieben mehrheitlich Bewohner von Agrarregionen mit schlechter Infrastruktur. Erst tragbare Transistorradios und der Einsatz von Maschinen in der Landwirtschaft begannen dies nachhaltig zu verändern. Über die ländliche Situation Mitte des 20. Jahrhunderts sagte Barth:

"The Kurdish villager is not concerned with abstract features of village organization - he sees the organization of his own villages, and other organizations affecting him, in terms of hierarchies of individuals, of named and familiar people, each with a familiar total status." (1979: 79). 
$\mathrm{Zu}$ der schlechten Infrastruktur gehörte auch, dass es bis in die 1980er Jahre in den von Kurden besiedelten Regionen kaum Industrie gab und die Gesundheitsversorgung notdürftig war. Nahezu 90 Prozent der Kurden waren noch immer in der Landwirtschaft tätig (YalçinHeckmann 1991: 89). Das Baath-Regime wollte die feudalen Strukturen der Kurden zugunsten der eigenen Verwaltung beseitigen und setzte diesen Willen in Form verschiedener Landreformgesetze, die auch das Weiderecht der Bauern betrafen, um (Deschner 2003: 234). Industrie gibt es nach wie vor abgesehen von Ölraffinerien wenig. In Sulaimaniya konnten sich Zigarettenfabriken etablieren und kleine Industrien für den täglichen Konsum. Seit 1991 gestaltete sich Arbil zum entwickeltesten Zentrum Kurdistans und auch Dahuk konnte neue industrielle Zweige erschließen (O’Shea 2003: 59). Heute leben von den etwa 3,5 Millionen Menschen in Irakisch-Kurdistan 2,5 Millionen in den urbanen Zentren Arbil, Sulaimaniya und Dohuk, vor allem infolge der Landflucht und Deportationen (Fischer-Tahrir 2003:24).

Bengio geht soweit, zu sagen, dass neben der Abwesenheit der Bagdader Zentralregierung in den letzten beiden Jahrzehnten die Modernisierung der kurdischen Gesellschaft die größte Kohäsionskraft für kurdische Identität geworden sei (2005: 176).

\subsection{Internationalisierung: Emigration und Diasporen}

Dass ein massives amerikanisches Interesse in den letzten Jahren zu der Gestaltung der kurdischen Autonomieregion beiträgt, ist kein Geheimnis und nicht nur, dass die Wetterdaten der kurdischen Regierungshomepage von der US-Air-Force geliefert werden, beweist dies. Den USA geht es beim Aufbau des kurdischen Bundesstaates auch um ein Aufbrechen des sogenannten schiitischen Halbmondes, der gedachten Achse von Hisbollah im Libanon über Iran nach Bahrain. Ohne auf die politischen Umstände näher eingehen zu wollen, lässt sich doch feststellen, dass bereits das internationale Interesse und die Weltöffentlichkeit die Begegnung von regionalen und globalen Lebenshorizonten der Menschen provozieren müssen. Außerdem ermöglicht die Internationalisierung des regionalen Konfliktes in zunehmendem Maße zu kurdischer Identität zu stehen, da sie überregional erlebt werden kann und die wichtige äußere Akzeptanz erfährt. In diesem Zusammenhang und in Zeiten moderner Kommunikationsmöglichkeiten muss sich der Blick zusätzlich auf die kurdischen Diasporen richten. ${ }^{30}$ Viele Migranten wirkten zu Zeiten osmanischer Vorherrschaft als oppositionelle Modernisierer in Europa, die sich für dezentralere Strukturen engagierten. Sie kehrten noch nach Zusammenbruch des Reiches zurück (Emanuelsson 2005: 65). Arbeitsemigration aus

\footnotetext{
${ }^{30}$ Die verschiedenen Diasporen können im Rahmen dieser Arbeit zwar nicht ausführlich erfasst werden, zumal auch Studien in diesem Bereich noch unzureichend sind, dennoch soll auf diesen, die Gesellschaft im Irak zunehmend prägenden Aspekt hingewiesen sein.
} 
den kurdischen Gebieten hatte im 20. Jahrhundert vor allem in Richtung der Städte Istanbul, Teheran und Tabris stattgefunden. Die Migrationsursachen wandelten sich in der zweiten Hälfte des 20. Jahrhunderts. Unfreiwillige Massenmigration war Folge der massiven Kriegszerstörungen kurdischer Dörfer (Bruinessen 1999: 2).

\begin{abstract}
„The stateless and divided Kurdish 'territorial minority' forms a diaspora (extra-territorial minority) consisting of immigrants and refugees from four 'countries of origin' spread throughout many nation states. Besides the internal Kurdish interactions, the Kurds are surrounded by the political agenda and activities of other actors at the sub-state, state and supra-state levels (...) On a more general level, despite the fact that the Kurds constitute a relatively large diaspora consisting of two or ten million depending on definition there is a lack of comprehensive study on Kurds who have sought refuge or migrated." (Emanuelsson 2005: 20f.).
\end{abstract}

Schätzungen des kurdischen Instituts Paris gehen von etwa 1,2 Millionen Kurden verschiedener Herkunftsländer in Westeuropa aus, davon allein 600000 in Deutschland. ${ }^{31}$ Die Erfahrung einer derartigen Diasporagröße verändert den territorialen Bezug. Der Boden war für viele nicht mehr der Ort der permanenten Wiederkehr. Der Kontakt mit Kurden, die sich in der Diaspora unter anderen sozio-politischen Umständen organisierten, unterstützte die Akzeptanz verschiedener Identitäten (Emanuelsson 2005:18). Dazu bedenke man, dass dies Potential brachte, eine starke kurdische Identität zu schaffen. Denn in der Diaspora gab es "those with one or more Kurdish grandparents, and those with more distant Kurdish ancestors. Nothing prevents these more peripheral Kurds from discovering and emphasising their kurdishness in certain contexts." (Bruinessen 1999: 11). Mit Abnahme der Kriegshandlungen konnte sich der Austausch in den vergangenen 20 Jahren intensivieren. " $A s$ the Kurds became less like refugees in the 1990s in the sense that they were increasingly able to travel, return and circulate, they accommodated the views of the Kurdish parties present on the ground in Iraqi Kurdistan."(Emanuelsson 2005: 217).

\title{
3. Integration nationalstaatlicher Strukturen und Ideale
}

Die Adaption des nationalstaatlichen Modells als Ideal gesellschaftlicher Ordnung lässt sich lange vor der Errichtung der kurdischen Autonomieregion im Irak belegen. Denn mit Ausrufung der kurdischen Republik Mahabad (1946) wurde eben auch die Akzeptanz von Nationalstaatlichkeit als Repräsentation für ethnische Identität zum Ausdruck gebracht. Die Gesellschaft für die Wiedererweckung Kurdistans (KOMALA) hatte seinerzeit bereits den Vereinten Nationen einen detailliert erstellten Plan über die Staatsgründung übergeben.

Der deutliche Beweis dafür, dass das Konzept des Nationalstaats die kurdische Gesellschaft erreicht hat und einen realen Bezugsrahmen für das Stiften kollektiver Identität bietet, ist die

\footnotetext{
${ }^{31}$ http://www.institutkurde.org/kurdorama/ (abgerufen am 18.05.09)
} 
Tatsache, dass heute der Bundesstaat Kurdistan im Irak im Gegensatz zum übrigen Land wirtschaftlichen Aufschwung erlebt und eigene Parlamente mit eigener Verfassung bildet. Unmittelbar nach der langjährigen osmanischen Herrschaft stellte deren hinterlassene politische Struktur noch eine Hürde für nationale Identifizierung und Erhebung dar.

\begin{abstract}
„Es war problematisch die unpolitisierten Massen in bestimmte Bahnen zu lenken und den kurdischen Nationalgedanken unmittelbar nach dem Krieg entscheidend ins Bewusstsein zu rufen. Zudem waren diese Strömungen nicht einmal untereinander über ein gemeinsames politisches Handeln einig. Die Bemühungen solcher Kräfte fanden zu wenig Resonanz in der Bevölkerung. “(Asadi 2007: 76).
\end{abstract}

Ein erster Höhepunkt kurdisch-nationalen Strebens war das kurzlebige kurdische Kabinett, das Scheich Mahmud im November 1922 zum kurdischen König ernannte (Asadi 2007: 102). Obwohl der Vertrag von Lausanne grundsätzlich um die nationale Integration der Kurden bemüht war, gab es nur kleine Erfolge, indem einige Staatsämter mit Kurden besetzt wurden.

Die Realisierung kurdischer Selbstverwaltung nach nationalstaatlicher Manier schien noch bis in die jüngere Vergangenheit ausgeschlossen (Behrendt 1993: 401). Jedoch zeigen die Entwicklungen der vergangenen Jahre genau dies. Mit Blick auf die Intransparenz der Parteienzulassungen kann der nationale Charakter nicht als demokratisch beschrieben werden.

\title{
3.1 Nationale Strukturierung und Institutionenbildung
}

Insofern den Kurden eine adäquate politische innere und äußere Struktur sowohl als Folge des britischen Diplomatie-Prinzips wie auch der Ideologie der irakischen Regime verwehrt war, beschränkt sich die Institutionalisierung auf die vergangenen zwei Jahrzehnte.

Kurden, die zwar im eigenen Land von zentralen Organen ausgegrenzt waren, kannten allerdings Aspekte nationaler Repräsentation. Sie verfügten über para-diplomatische Vertretungen in der Türkei, Iran, Frankreich, England und den Vereinigten Staaten. Jenen fehlte es zwar an formalem Diplomatenstatus, aber sie konnten an wesentlichen Entscheidungen mitwirken und für die eigenen Interessen kämpfen, als Bagdad selbst keine Vertretungen in den Ländern hatte (Bengio 2005: 179).

Nach dem Errichten der Schutzzone und der Wahl im Jahr 1992 einigten sich die beiden siegenden, regional verorteten Parteien (vgl. Anhang V) auf eine Fifty-Fifty-Lösung, bei der jedes Ministerium und jedes Amt doppelt besetzt wurden, womit sich die Kontrahenten jedoch gegenseitig lähmten. Ein weiteres Problem war die finanzielle Ausstattung. Das einzige Einkommen in der Region stammte aus dem Schmuggel und von einzelnen Hilfsorganisationen, die durch den Anspruch der gleichen Verteilung ihrer Mittel geschwächt waren (Rogg 2003: 14). Dem folgte, dass es nicht nur zwei Flaggen, sondern auch zwei 
Judikativen und Exekutiven und Armeen gab, also ein Talabanistan und ein Barzanistan

(Bengio 2005: 180). Vor allem aber mangelte es an einer kurdischen Zivilgesellschaft.

„The executive governmental structures have matured considerably under the divided political system. Despite needing twice as many staff as would be necessary if there were only one $K R G$, one of the silver linings of this situation is that twice as many bureaucrats have been exposed to the experience of governance, UN interactions, and NGO assistance. The Kurds had very little numbers of bureaucrats with appropriate experience in 1991, but they now have a substantial body of trained administers. The capability of Kurds to govern their own country has obviously been enhanced by these actions." (Stansfield 2005: 204).

Neben der „Massenproduktion“ von Bürokraten und Institutionen in Abwesenheit des irakischen Staates war ein Ausgangspunkt für das erfolgreiche Errichten eigenständiger nationaler Strukturen das Oil-For-Food-Programm, welches durch die Resolution 986 der Vereinten Nationen ins Leben gerufen worden war. Zwischen 1997 und 2002 erhielt das autonome Kurdistan hieraus 4,8 Milliarden Dollar. Daneben bezieht die junge Verwaltung Geld aus Zöllen und Gebühren der Erdölpipelines (vgl. Anhang VI). Die Schaffung der Freihandelszone schuf weitere Einnahmequellen zur Finanzierung des Staatsapparates (Heintze 2003: 451). Zentral sind nun die jungen Verfassungstexte des Iraks. Die irakische Verfassung, die im Oktober 2005 nach einem landesweiten Referendum angenommen wurde, bemüht sich, die gesellschaftliche Fragmentierung $\mathrm{zu}$ würdigen und den verschiedenen ethnischen Identitäten mit Minderheitenrechten, Quotierungen, und Proporzsystemen zu begegnen (Eichhorst/Sinjen 2006: 405). In dieser Interimsverfassung besteht das Kurdische als offizielle Sprache neben dem Arabischen und es gibt ein klares Bekenntnis zum Föderalismus. Nachdem sich 2003 die kurdischen Parteien auf die administrative Zusammenlegung der Regionen Suleimaniya und Arbil einigten, bekannten sie sich ihrerseits in der nach den Wahlen 2005 angenommenen Verfassung der Region Kurdistan dazu, Teil des föderalen Iraks zu sein (Asadi 2007: 501). Daneben weist sie den Bundesstaat als demokratisch, parlamentarisch und religiös tolerant aus. All diese Entwicklungen, so meint Stansfield, haben dazu geführt, dass die Kurden nun Regierung synonym zu Kurdistan verwendeten. ,, The people of Kurdistan (or, at least the Kurdish majority there), long distinct in their ethnic identity, have developed a vibrant Kurdish nationalism." (2005: 205).

\subsection{Nationale Symbole und Rituale}

Als da konstruktivistische Ansätze davon ausgehen, dass abstrakte Größen wie Macht und Herrschaft nur in Form von Symbolen kommuniziert werden können, muss berücksichtigt werden, dass diese Symbole von Menschen geschaffen und somit gezielt zum Einsatz gebracht werden. Politik wird mithilfe von Symbolen gemacht (Heidemann 2003: 168). 
„Kurdistan's population has become considerably more homogenous over the last century, probably reinforcing the formation and maintenance of a Kurdish identity in Kurdistan. Yet the impetus, largely from the Kurdish diaspora, towards a secular nationalism has led to the necessary exaggeration of the harmony that existed between Kurdistan's mosaic of inhabitants." (O’Shea 2003: 190)

„A mental image of ethnic and national identity must be constructed artificially, by which means the political community can be imagined and experienced by its putative inhabitants " (O'Shea 2003:149).

Da sich Kurden abgesehen vom kollektiven Namen auf keine Anerkennung oder Darstellung als geschlossene homogene Entität stützen können, müssen kollektivierende Symbole geschaffen werden. Dazu kann die intensiv betriebene Sprachförderung dienen.

\begin{abstract}
„The Kurdish regional identity has taken substantive and symbolic forms. The Kurdish language has been developed and employed in the public sphere, including schools, universities, the administration, and broadcasting and print media. There has been widespread development and display of national symbols, such as Kurdish flags, a Kurdish hymn, and the erection of statues and portraits of Kurdish heroes, such as Mustafa Barzani and Mahmud Barzinji." (Bengio 2005: 176).
\end{abstract}

$\mathrm{Zu}$ einer einigenden und beliebten Identifikationsfigur mit dem nationalstaatlichen Kurdistan scheint sich Saladin, der kurdisch-stämmige Ayyubide des 12. Jahrhunderts, zu mausern.

Am symbolträchtigsten ist die kurdische Hymne und Flagge (siehe www.krg.org). Als die Briten Scheich Mahmud infolge kurdischer Aufstände 1922 die Rückkehr gewährten, hisste jener die kurdische Flagge zum wiederholten Male (Asadi 2007: 101). Auch das Hissen bei gleichzeitigem Einholen der britischen Fahne weist darauf hin, dass schon früh eine Identifikation mit nationalstaatlicher Symbolik und Ideologie möglich war (Asadi 2007: 49).

\title{
3.3 Politische Repräsentation durch Parteien
}

Obwohl Irakisch-Kurdistan sich um tragende nationalstaatliche Pfeiler zu bemühen scheint, muss doch das hierfür nötige Parteienwesen als Achillesverse der Region gewertet werden. Nicht als Ausdruck von Demokratie sondern vielmehr verbunden mit schmerzlichen Erfahrungen des Bürgerkriegs stehen die wichtigen kurdischen Parteien einander gegenüber.

Versuche entsprechend schichtübergreifender Organisation gab es dabei sehr wohl frühzeitig.

„At the End of World War I. the organisation (renamed the society for Rise and Progress of Kurdistan) reopened in Constantinople. It was still led by the old notables but it began to also involve Kurdish workers, peasants and others with tribal origins. Clearly they had developed ideas and networks in which the kurdish nation was conceived in territorial terms. The organisation presented a single proposal at the International Peace Conference in Paris in which Wilson's Program for World Peace was discussed." (Emanuelsson 2005: 65).

Neben den von Stammes- und Militärführern forcierten Zusammenschlüssen entwickelten sich seit den dreißiger Jahren politische Bewegungen gemeinhin auf demokratischer Basis. Gewissermaßen als Abwehrreaktion auf den Panarabismus gründeten bürgerliche Kurden die 
Vereinigung Hoffnung (Hiwa) mit geringer öffentlicher Präsenz. Hingegen forderte die Partei Befreiung (Rizgari) in ihrem Programm offen die kurdische Unabhängigkeit. Solche Gruppierungen waren allerdings weder von langer Dauer noch konnten sie eine größere Anzahl von Kurden mobilisieren. Dagegen sammelten sich Kurden zahlreich in der kommunistischen Partei Iraks (Strohmann 2003: 123). Unter den kurdischen Studenten mit Kontakt zu linken Ideen trat der Jurist Ibrahim Ahmad in den dreißiger Jahren besonders hervor. Er verbreitete in vielen Publikationen kommunistisches Gedankengut. In einem Buch von 1937 „, Kurden und Araber“ forderte er das Recht auf Selbstbestimmung ein und wurde verhaftet. Während der Wirren um Mahabad setzte sich eine eigene kurdisch-kommunistische Partei ab, die Mustafa Barzani zu ihrem Präsidenten wählte. In der illegal verteilten Zeitung forderte sie neben besseren Bildungschancen und Mindestlöhnen die Schaffung einer autonomen Region Kurdistans. Echte separatistische Tendenzen setzten sich hier nicht durch (Günther/Brentjes 2001: 38f.). Bald schon ließ die irakische Regierung jegliche Opposition im Land unterdrücken, verbot Parteien und verhaftete deren Führer. So gab es andere Formen des Widerstands, beispielsweise die Gründung einer Anwaltsliga, die Bauern gegen ein 1951 erlassenes Gesetz über die Beurkundung von Eigentumsrechten vor den Gerichten verteidigte. Ihre Tätigkeit brachte die Regierung dazu, dieses Gesetz außer Kraft zu setzen (Günther/Brentjes 2001: 40). Schon zu dieser Zeit wuchsen Spannungen zwischen den verschiedenen Flügeln der Parteireste. In Barzanis Abwesenheit gelang es Ibrahim Ahmad, dem Schwiegervater Jalal Talabanis, Generalsekretär der jungen KDP zu werden und linke Programmatik umzusetzen, was die Partei für Bauern attraktiver machte und zunächst das sozialistische Regime von Qassim stützte. In der kommenden Phase, in der Qassim die beiden Seiten gegeneinander ausspielte, konnte Barzani nur kurzfristig zwischen „Stammes-Kurden“ und den Anhängern Ahmads und Talabanis Ruhe schaffen. Die KDP erlebte 1964 die Gründung eines neuen Politbüros unter Talabani, der sich 1966 komplett abspaltete (Strohmann 2003: 128). Um 1970 näherte sich die KDP einerseits der Baath-Partei an, um ihren Alleinvertretungsanspruch für Kurden geltend zu machen (Asadi 2007: 297). Andererseits zeigte sich Barzani versöhnlich durch Amnestiegewährung für die Gruppe um Talabani und die Umgestaltung der Parteistruktur, um den veränderten sozialen Verhältnissen gerecht zu werden (Sharif 1991:141). Als dann die Gewalt gegenüber den Kurden einen Höhepunkt erreichte, kam es zum endgültigen Bruch im Parteienspektrum. Geflohene Führer, darunter Jalal Talabani, gründeten formal 1976 in Westberlin die Patriotische Union Kurdistans. Aufgrund von Verfeindungen, besonders mit Mustafas Sohn Idris Barzani, war das Verhältnis umso schlechter (Günther/Brentjes 2001: 45). Abgefallene Kämpfer sowohl 
der KDP und PUK gründeten 1979 die Sozialistische Partei Kurdistans. Eine Übersicht zur Veranschaulichung der Parteienlandschaft um 1980 befindet sich im Anhang VIII.

Mit Entfernung der irakischen Zentralautorität entschieden zu Beginn der 90er Jahre die kurdischen Parteien für schnellstmögliche Wahlen, um das Machtvakuum zu füllen. Diese Wahlen von 1992 wurden von Beobachtern auch als akzeptabel demokratisch bewertet (Emanuelsson 2005: 74). Im Wahlergebnis erzielten PUK und KDP, zwei der acht Parteien, gemeinsam über 80 Prozent und zeigten sich willens gemeinsam zu regieren. Es wurde ein Regionalparlament und eine Regionalregierung geschaffen. Probleme waren jedoch vielfältig. Die die benachbarte Türkei provozierende Akzeptanz der türkischen PKK, ökonomische Krisen und ungeklärte menschliche Schicksale, sowie die ideologische Taktiererei der Nachbarländer belasteten das Regierungsvorhaben (Salih 2005: 136). Vor Inkrafttreten des Oil-for-Food-Programms war die Region zudem wirtschaftlich isoliert.
„Unter diesen Bedingungen erweisen sich alle Versuche der Selbstorganisierung und Selbstverwaltung als extrem schwierig. Wichtigster Arbeitgeber in Südkurdistan ist die Regionalregierung, die aber schon die Hälfte des eigenen Haushalts für die Gehälter der 30000 Bewaffneten, Lehrer und Angestellten aufwenden muss. (...) Angesichts einer Arbeitslosigkeit von 70-80\% der arbeitsfähigen Bevölkerung kam es in Südkurdistan in dem selben Jahr zur Gründung einer ,,Union der Arbeitslosen. “(Günther/Brentjes 2001: 131).

Die Schaffung überregionaler Strukturen scheint in den 90er Jahren auch daran gescheitert zu sein, dass die Parteiführungen nicht bereit waren, an gewählte Vertreter Macht abzugeben, oder sich selbst in demokratische Strukturen einzugliedern, obwohl sie zuvor gleichermaßen verschiedene Wählerschichten anzusprechen schienen (Strohmann 2003: 132-140).

„, In such a situation, the Kurdish parties developed several areas of conflict. Chief amongst them was the question of how customs revenues, the only income of the administration, should be shared. Eventually, this led to an internal war between 1994 and 1998, to two parallel and competing Kurdish administrations and to increased political and economic insecurity and the ensuing flight from and within the safe areas. (...) Stansfield concludes that activities by Barzani (KPD) and Talabani (PUK) should not be characterised as activities by the tribal dictator and the mob as such tendencies made the situation more problematic in an already difficult regional geopolitical situation." (Emanuelsson 2005: 75-77).

Die Parteien waren langsam bei der Umsetzung des Washingtoner Abkommens von 1997. Dies enthielt die Aufforderungen zu Gefangenenaustausch, Rückführung umgesiedelter Familien sowie gemeinsamer Verwaltung kurdischer Gebiete. Erst im Januar 2001 einigten sich die Anführer der Parteien auf vereinfachte Bewegungsfreiheit der Menschen und Verringerung der militärischen Präsenz entlang früherer Kampflinien (US Congress 2001).

\subsubsection{Die KDP (Kurdish Democratic Party)}

Die Demokratische Partei „,war ursprünglich eine Ansammlung aller möglichen Strömungen, die für die kurdische Sache eintraten: Rechte wie Linke, progressive wie konservative, 
reformistische wie revolutionäre Kräfte." (Asadi 2007: 362). In Folge der Märzniederlage 1975 war der neue Kurs anti-imperialistisch gegen die USA aber auch den Iran ausgerichtet. Selbst der legendäre Parteichef Barzani blieb von Kritik nicht verschont, als sich die Situation mit der Gründung der PUK zuspitzte. Dessen Sohn Masud Barzani, zuvor Leiter der kurdischen Geheimdienstorganisation, bemühte sich zusammen mit den anderen führenden Köpfen der KDP um eine fortschrittliche und linksorientierte Neuausrichtung. Die Partei bekannte sich durchgehend zur kurdisch-arabischen Zusammenarbeit innerhalb des Iraks und zeitweise waren Parteigänger als Minister im irakischen Kabinett vertreten (Deschner 2003: 241). Mustafa Barzani, der den Beinamen Mulla als Symbol seines geistlichen Strebens erworben haben soll, war nach den von ihm geführten Aufständen auch als Stammesführer aktiv. Als solcher schlug er 1959 die Zibari, die alten Rivalen der Barzani (vgl. Anhang II). Sein Bruder Ahmad war der eigentliche Führer der Barzani und wurde vor allem wegen der Abkunft von einer Sufi-Familie verehrt ${ }^{32}$ (Hottinger 1965: 469). Nach Mustafa Barzanis Rückkehr in die kurdische Politik 1958 wurden zunehmend auch traditionelle Stammesführer in der Partei aktiv (Sarbest 2001: 32). Mustafa Barzani flüchtete nach seinem gescheiterten Widerstand in den Iran und weiter in die USA, wo er 1979 starb (Sharif 1991: 146). Die Parteiführung der KDP sah sich danach mit bitteren Spaltungsversuchen konfrontiert, wurde letztlich von Masud und Idris Barzani weitergeführt und stand weiterhin den Stammesführern näher als andere Parteien. Welchen Stellenwert noch immer die traditionelle Stammesordnung hat, zeigt der aus der Heirat Mustafa Barzanis mit der Tochter eines Zibari-Aghas in den 1940er Jahren hervorgegangene Sohn Masud (geb. 1946). Dieser bleibt ein wichtiges politisches Bindeglied zwischen den Stämmen (Franz 1986: 105-107). Die Parteistruktur ähnelt bis heute der einer Kaderpartei des ehemaligen Ostblocks. Sie wird angeführt von einem 21-köpfigen Zentralkomitee, aus dem ein 8-köpfiges Politbüro hervorgeht.

\subsubsection{Die PUK (Patriotic Union of Kurdistan)}

Bis 1975 war Mulla Mustafa Barzani mehr oder weniger eine Projektionsfläche für ein kollektives Gefühl, das die Mehrheit der Kurden zusammenhielt. Doch die Niederlage von 1975 schuf neue verwirrende Konstellationen, neue politische Strömungen (Asadi 2007: 484). Obwohl Barzani mit Kongressen und der Errichtung verschiedener neuer Gremien auf die Unzufriedenheit reagierte, wurde die innere Spaltung zum Flächenbrand. Darüber hinaus besaß niemand ein einheitliches Konzept von dezentralen Strukturen und Machtverteilungen. „Die Unstimmigkeit unter den Kurden hinterließ einen tiefen Riss in der kurdischen

\footnotetext{
${ }^{32}$ Hierbei sind die elterlichen Verhältnisse nicht ganz deutlich.
} 
Gesellschaft, der bis heute andauert. “ (Asadi 2007: 476). Vorläufer der Patriotischen Union Kurdistans verfügten in den Ereignissen der vom Baath-Regime deklarierten Autonomie noch über keinerlei Schlüsselpositionen.

Als Jalal Talabani im Mai 1975 die PUK ins Leben rief, verfolgte sie ebenso wie die KDP das Ziel der Demokratie für Irak und Autonomie für Kurdistan. Die PUK gründete sich aber mit marxistisch-leninistischer Programmatik und machte die feudalistisch-tribalistische Führung der KDP verantwortlich für das Scheitern der Aufstände (Asadi 2007: 360). Der PUK schlossen sich sozialistische und marxistische Gruppierungen an, die gemeinsam nach der Rückkehr Talabanis im Juli 1977 zusammentraten (Hottinger 1965: 473f.).

$\mathrm{Zu}$ den Gründungsmitgliedern zählten einige Intellektuelle, vor allem Literaten, Wirtschaftswissenschaftler und Juristen, wie Nauschirwan Mustafa oder Fuad Masum. ${ }^{33}$ Sie verfügten über gute internationale Beziehungen. ${ }^{34}$

\subsubsection{Elitenrekrutierung}

Nachdem die Zentralisierungsversuche offenbar die neue politische Ordnung prägten, in der Scheichs neben Stammesführern als Machthaber erschienen, ist unübersehbar, dass die heutigen kurdischen Repräsentanten mit dieser Entwicklung noch immer in engem Zusammenhang stehen (Emanuelsson 2005:64). Es ist wohl kaum ein Zufall, dass Talabani nach dem Sturz Husseins Präsident des Iraks wurde und Barzani Ministerpräsident IrakischKurdistans.

Wenn der Name Scheich Mahmuds in einem assoziativen Zusammenhang mit der Entstehungsgeschichte der Kurdenfrage steht, so ist die Weiterentwicklung dieser Frage mit dem Namen Mustafa Barzani verbunden. Beide zeichneten sich durch die Stammeszugehörigkeit aus und traten mit einer bestimmten Gefolgschaft an (Asadi 2007: 138). Hartnäckig scheinen sich Konzepte von Macht, wie schon Yalçin-Heckmann sie für die Hakkari-Führer beschrieb, auf traditionelle Machtressourcen zu stützen:

"Coercion, confiscation of land and animals, the use of sheer violence against the central government's administrators or local opposition trough hirelings are as much a part of the agha politics as consolidating their position trough affinal links to rival aghas, forming kirve relations with the local military officers, supporting the government party as well as Kurdish nationalist politics and having professionals, politicians and farmers simultaneously in the family." (1991:131).

Es ist auch offensichtlich, dass trotz zunehmender Unabhängigkeit politische Entscheidung ein Privileg blieb, nämlich eines der Anführer der KDP und der PUK, die infolgedessen auch

\footnotetext{
${ }^{33}$ Für weitere Informationen siehe Franz 1986.

${ }^{34}$ Ein Blick auf die mehrsprachige Homepage spiegelt diesen Eindruck wieder (siehe http://www.puk.org/)
} 
ohne jegliche demokratische Kontrolle getroffen wird. Im Resultat verstärkte sich die Abhängigkeit der Bevölkerung von den Parteien, von den städtischen und Stammesführern sowie von den internationalen Hilfsorganisationen (Günther/Brentjes 2001: 132).

Die Rolle der Frau innerhalb der sich neu entwickelnden Strukturen bedürfte einer umfassenderen Analyse, für die hier zu wenig Material vorliegt. Frauen haben vielfach Rollen außerhalb des ihnen zugewiesenen Platzes innerhalb des Hauses eingenommen. Töchter aus höheren Schichten reisen vielfach zum Studieren in andere Länder (Fischer-Tahrir 2003: 31). Im neuen Parlament gibt es eine Frauenquote von 25 Prozent, bei deren Besetzung viele der bereits durch die Betrachtung der beiden großen Parteien bekannten Namen auftauchen. ${ }^{35}$

\subsection{Nationalisierung durch andere politische Organisationseinheiten}

Obgleich Nichtregierungsorganisationen keine unmittelbar vom Nationalstaat abhängigen Einheiten sind, tragen sie doch ein entsprechendes Verständnis von Demokratie und Menschenrechten in die Einsatzorte. Sie helfen beispielsweise bei der Aufklärung der Ermordungen und bei der Witwenbetreuung, getragen von einem humanistischen Ideal. Entsprechendes gilt auch für kurdische Exilorganisationen:

"They expressed support for the establishment of rule of law and democratic systems in the region of origin. They considered democratic structures as well as agency from below as necessary components of democratic systems. They regarded democracy as a proper means for the promotion of peaceful political processes and relationships between people with different options.(...) Whilst some were more successful in building networks with local/national NGOS, several could be characterised as actually belonging to the transnational/global network of (I)NGOs concerned with issues such as human rights, development and racial discrimination. (...) To some extent, they also met with other diaspora organisations." (Emanuelsson 2005: 210-212).

Mittlerweile gibt es mehr als 50 bekannte Organisationen beziehungsweise Parteien, die von verschiedensten Interessen aus verschiedenen Gründen finanziert werden.
„The matter of asymmetry related to the Kurdish actors is obvious when considering different organisation's access to financial and other kinds of support provided by Western authorities and governments. Although the organisations in Germany finally succeeded after a long struggle to establish Kurdish education and broadcasting in Germany, they still suffered from discontinuous funding and non-recognition. (...) In addition, in all European countries the Kurdish organisations faced racism, xenophobia, unemployment and the segregation of Kurdish migrants/refugees" (Emanuelsson 2005: 219f.).

Was Frauenorganisationen betrifft, so schlossen sich Frauen politisch eher den kommunistischen Parteien an. Dazu stand Studentinnen und Akademikerinnen vor allem die zur KDP gehörende Frauenunion Kurdistans (Areftan) offen, die bereits 1952 gegründet

\footnotetext{
${ }^{35}$ vergleiche hierzu auch die nach Parteien geordnete Liste der Parlamentsmitglieder auf: http://www.krg.org/uploads/documents/KNAMembers_2006_11_30_h12m44s8.pdf
} 
worden war. Eine untergegliederte Gruppe der irakischen Frauenliga mit kommunistischem Profil entwickelte sich seit den 1960er Jahren. Eine später der PUK zugehörige Frauenorganisation (Kurz: Jinan) ${ }^{36}$ wurde 1989 im iranischen Exil von weiblichen Führungskräften gegründet. Parteiunabhängige Organisationen entwickelten sich erst in den 90er Jahren. Diese begannen auch unangenehme Themen wie die Mädchenbeschneidung zu thematisieren und forderten das Sanktionieren von Ehrenmorden (Fischer-Tahrir 2003: 3335). In den letzten Jahren konnten Frauengruppen mit Lobbyarbeit Erfolge verzeichnen, wie eine Änderung des Personenstandrechts und die Errichtung eines Frauenhauses, sowie die Abnahme der Ehrenmorde (Rogg 2003: 18).

\subsection{Peschmerga}

Kampfeinheiten finden in den sozialwissenschaftlichen Betrachtungen kurdischer Gesellschaft kaum Berücksichtigung, obwohl die Existenz und Formierung von Milizen den Lebensalltag stark prägten und sie ihn als Militär des Bundesstaats Kurdistan prägen. Die zunehmende Bedeutung des Soldatenstatus erwirkte zudem eine weitere gesellschaftliche Partizipationsform inmitten der zerrütteten Strukturen. Die historischen Wurzeln der sogenannten Peschmerga reichen zurück bis in die Zeit des Unterganges des Osmanischen Reiches und der Qadscharendynastie. Der Begriff Peschmerga wurde allerdings erst von dem bereits erwähnten Mitbegründer der KDP Ibrahim Ahmed geprägt. Seither hat sich der Name als Synonym für die meisten Kämpfer der kurdischen Parteien und Organisationen etabliert. Damit kann er sich also auf die Kombattanten von ideologisch völlig unterschiedlichen Parteien beziehen. Peschmerga bekamen große Aufmerksamkeit in den schweren Auseinandersetzungen der 1970er Jahre, wobei sie verschiedentlich auf ausländische Rüstung angewiesen waren (Asadi 2007: 354). Die Peschmerga galten lange als Guerillaarmee von Barzani, ihrem formalen General, der zahlreiche Lager und Ausbildungsstätten unterhielt (Deschner 2003: 239-241). Später umfassten sie allgemein auch die verfeindeten, bewaffneten Einheiten der KDP wie PUK.

Als Kurden 2003 die US-geführte Operation Iraqi Freedom unterstützen, wurde davon ausgegangen, dass Barzani und Talabani gemeinsam rund 80000 Soldaten gegen Bagdad schicken könnten (Heintze 2003: 455). Um das irakische Militär mit zwei Fronten zu überfordern, kämpften Truppen der PUK in Mosul und Kirkuk. Indes wurden Peschmerga bei ihrem Angriff auf Camps der islamistischen Gruppe Ansar al-Islam in den Bergregionen unterstützt (US-Congress). Die Peschmerga, die in Regionalgarde umbenannt wurde und als

\footnotetext{
${ }^{36}$ Die Benennung der Gruppen bezieht bereits verschiedene regionale Dialektpräferenzen mit ein und war im Zuge des Versuchs der Vereinheitlichung ein wichtiger Diskussionspunkt.
} 
nordirakische Armee nicht mehr den einzelnen Parteien unterstellt sein soll, darf sich heute einseitig im übrigen Irak bewegen und hat 2007 die Sicherung der drei kurdischen Provinzen komplett von der US-Armee übergeben bekommen (Asadi 2007: 502). Nicht nur die großen Verluste junger Männer führten dazu, dass Frauen bei der Versorgung der Peschmerga aushalfen und gelegentlich sogar als Kämpferinnen aktiv wurden (Fischer-Tahrir 2003:31).

Über die Stärke der Peschmerga gibt es widersprüchliche Angaben. Für die vergangenen Jahre war die Anzahl der Mitglieder der verschiedenen Kampfverbände etwa konstant. Die PUK verfügte in etwa über 10000 Kämpfer, die KDP über 15000 und das Irakische Militär über etwa 350000 (vgl. Daten des US-Congress).

\subsection{Irakisch-Kurdistan: Eine Absage an Groß-Kurdistan?}

„, Maps of Kurdistan must reflect a concept and a desire rather than reality“(O’Shea 2003: 194).

Dass es während der Etablierung eines kurdischen Staatsgebildes im Irak neben einigen Stimmen keinen deutlichen Diskurs über den aufrechterhaltenen Wunsch des Zusammenschlusses zu einem Groß-Kurdistan gibt, hat viele Ursachen, die sich im Wesentlichen mit dem Verweis auf den internationalen Charakter der Entwicklungen beschreiben lassen.

Ohne dass es zuvor jemals ein geeintes Kurdistan oder klare Grenzen gegeben hätte, setzten sich konservative Kräfte für ein Zusammengehen mit den übrigen Kurden, ungeachtet sprachlicher oder anderer kultureller Barrieren, ein. Das frühe Bekenntnis der KDP zum Irak schuf somit den Grundstein für einen internen Dauerkonflikt (Bahjat 2001: 32). Bei der Einigung 1992 lagen die Vorstellungen der führenden Parteien nicht fern voneinander. Die KDP war offiziell für eine Autonomie Kurdistans in einem demokratischen Irak und die PUK für Autonomie in einem föderalen Irak (Strohmann 2003: 139).

Ein überaus wichtiger Faktor, der einem Zusammenschluss der von Kurden bewohnten Gebiete im Wege steht, sind die Interessen der betroffenen Nationalstaaten.

Forderungen nach einer nationalen Einheit aller Kurden, wie sie Vereinigungen nach dem Ersten Weltkrieg vortrugen, sind leiser geworden (Ağuiçenoğlu 1997: 212). Zu den Ursachen, die während der Gründung der Nationalstaaten die Ausrufung Kurdistans verhinderten, wurde bereits Einiges gesagt. Unter den komplizierten Rahmenbedingungen ließ sich ein kurdischer Staat kaum verwirklichen und in der Folgezeit begann sich die Kurdistanfrage in komplexer Weise zu überlagern (Asadi 2007: 77). Es muss im Interesse der Nachbarstaaten gewesen sein, dass die kurdische Bewegung im Irak nicht erfolgreich war, um nicht für vergleichbare Bewegungen im eigenen Land zu motivieren. Demzufolge herrschte allgemeine Einigkeit in 
der Unterwerfungspolitik. "The Iraqi Kurds were viewed as a mounting problem. Iraq's Arab majority was anxious about the Kurds' increasing political power; fundamentalist Iran was concerned with the Kurds `secularism; and Syria was apprehensive about Kurdish nationalism spreading across its border." (http://acd.iiss.org). Der überregionale Charakter des KurdenKonflikts verschärfte auch zeitweise zwischenstaatliche Verhandlungen (Asadi 2007: 261).

Ein weiterer Aspekt mit Wirkung auf das kollektive Bewusstsein der irakischen Kurden war die Vielzahl kurdischer Organisationen mit enger Bindung an das (westliche) Ausland. Moderne Kommunikation ermöglichte einfachere Kontaktpflege dorthin, als zu Kurden in der Peripherie und anderen Staaten, zumal Kenntnisse über die Situation anderer Kurden auf unmittelbaren Kontakt mit Flüchtlingen, begrenzt waren (Yalçin-Heckmann 1991: 29).

Insofern die Vereinten Nationen noch immer auf dem uti-possidetis-Prinzip beharren, wird eine Abspaltung trotz intensiver Autonomiebestrebungen nicht von der internationalen Staatengemeinschaft gutgeheißen werden und kann daher für die irakisch-kurdischen Akteure keine ernst gemeinte Option sein (Heintze 2003: 454). Wesentlich kommt hinzu, dass postnationale Gesellschaftskonzepte über die vielen MigrantInnen längst Eingang bei Kurden gefunden haben und in der Lage sind, solche Forderungen obsolet erscheinen zu lassen.

\footnotetext{
„Although the Kurdish organisation (...) did not deliberately promote (political, cultural and social) isolation/separation of Kurds from the majority population in the countries of settlement/asylum, in the 1980s they were rather inward and exile orientated in terms of their activities and focus. (...) In 1989 and thereafter, their focus on (overcoming) internal homeland political disagreements through national and political unity became only one aspect among many in their increasingly expanded and specified agendas in terms of plurality of issues, activities and relationships. Successively, their contacts and networks broadened to involve many state and non-state actors alike. (...) The Kurdish organisations entered a progression from exclusive nationalism to successively accommodating what has been called plurinationalism. This means that idea went from proposing outright Kurdish independence in the region of origin to legal guarantees for universal human rights (for individuals, majorities and minorities) and democracy within the borders of each state of origin. " (Emanuelsson 2005: 210- 213).
} 


\section{Fazit}

\section{Die Bewertung der strukturellen Veränderung sozialer Ordnung}

"It is a matter of perceiving the nation state or for that matter a micro-region such as Kurdistan, as only one level amongst many others at which issues of concern should be considered. (...) In the case of Iraq, the Kurdish organisations placed their hope in the institutionalisation of a unified understanding of universal human rights and pluralism, together with Kurdish autonomy or a federal Iraq." (Emanuelsson 2005: 216).

Ausgangspunkt für die vollzogenen Betrachtungen ist die Feststellung, dass sowohl endogene, wozu primär die segmentäre Struktur der kurdischen Gesellschaft gehört, wie exogene Faktoren verhinderten, dass weder der charismatische Führer Mahmud Barzinji noch Mustafa Barzani in Süd-Kurdistan eine nationale Bewegung auf die Beine stellen konnten, die von den Staatsmächten derart ernst genommen worden wäre, dass ein nationales Kurdistan hätte ins Leben gerufen werden können. Sogar obwohl die entsprechenden Vorstellungen eines solchen Staatsgebildes hier bereits für die Zeit um den Ersten Weltkrieg belegt werden konnten.

Die Betrachtung kurdischer Ethnizität hat deutlich gemacht, dass Kurden keineswegs über zuverlässige, überregional bindende Marker verfügen. Insbesondere die starke Orientierung an der Bundesstaatlichkeit Irakisch-Kurdistans seitens der Parteien stützt die These, dass im Ringen um eine allgemein akzeptierte ethnische Identität aus zweckrationalen Überlegungen an der nationalstaatlich verstandenen Konzeption Irakisch-Kurdistans festgehalten wird. Die nötige Außenwahrnehmung und Akzeptanz ethnischer Zugehörigkeit findet hier in bislang ungewohntem Maße statt, zumal sie sich in relativ klaren Grenzen vorfinden lässt. Diese Sichtweise berücksichtigt natürlich unzureichend den schwelenden Konflikt der Grenzregionen und den möglichen Druck konservativer Kräfte, aber entsprechend dem Sinne der Definition von Ethnizität, wie sie in dieser Arbeit verwendet worden ist, wird doch deutlich, dass die Anlehnung an moderne Staatlichkeit in weitaus größerem Umfang als die ideelle an ein fiktives Groß-Kurdistan für die Fremdwahrnehmung von Bedeutung ist. Die zunehmende Integration in den kapitalistischen Weltmarkt während der letzten Jahre ist mit Bezug auf den Theorieteil ein wichtiger Aspekt dieser Anerkennung nationaler Identität.

Das Erleben der ethnischen Identität (gegenüber möglichen anderen sozialen Identitäten) hing für Kurden vielfach mit gesellschaftlicher Unterdrückung und mit Verfolgung zusammen. Im Zuge der Assimilierungsversuche und des Ethnozids wurde die ethnische Identität der Kurden vor allem von den zentralen Regierungsinstanzen in den Vordergrund gedrängt, ohne dass ein klares Bekenntnis zu dieser hätte eingefordert werden müssen. Das parallele Erleben der grundsätzlichen Zurechenbarkeit zu einem größeren kurdischen kollektiven Gebilde und die Existenz als marginalisierte staatliche Minderheit musste die Orientierung an eben dieser 
Staatlichkeit mit sich bringen, da kaum Alternativen für die Gruppenorganisation denkbar waren. Außerdem war gerade „,das Identitätsbewusstsein dann groß, wenn Benachteiligungen der eigenen Gruppe wahrgenommen wurden. “ (Özdemir 2006: 92). Die Umsetzung der Adaption nationalstaatlicher Konzepte fand im Irak unter langanhaltenden Krisen statt.

Als der internationale Druck zu Beginn der 1990er Jahre den Weg geebnet hatte, Kurden sich selbstständiger verwalten zu lassen, tauchten nicht nur die ökonomischen Schwierigkeiten auf, sondern vor allem stürzte die Unerfahrenheit im Umgang mit Selbstverwaltung die Region in einen verlustreichen Bürgerkrieg. Der lange Status der Kolonie und Fremdbestimmung hat es formal an Übung im Verwalten staatlicher Strukturen fehlen lassen. Die dynastischen tribalen Strukturen waren kaum Veränderungen während des gesellschaftlich so viel verändernden 20. Jahrhunderts ausgesetzt gewesen.
„Dynastic organization was an essential tool for the imperial powers to exploit the Kurdish emirates, and this also stunted social development in Kurdistan, creating a highly stratified society and increasing the dominance of certain classes and tribes over the cultivators, ordinary towns-people and other tribes" (O'Shea 2003: 92).

Der erfolgreiche soziale Aufstieg der Scheichs parallel zum Zerfall des Osmanischen Reiches begründete sich darin, dass sie weit genug außerhalb der Stammesorganisation standen,

„, in der die gesellschaftlich dominante Schicht der Stammesleute nach dem Verschwinden der Emiratsstrukturen unterzugehen drohte. Gleichzeitig waren sie akzeptierter Teil des Systems (...) und konnten durch geschickte politische Allianzen und Heiraten die militärisch potenten Stämme an sich binden und allmählich wieder eine gewisse gesellschaftliche Stabilität etablieren. "(Behrendt 1993: 181).

Die traditionellen Bindungen der Stämme an die Aghas und Scheichs, die Religiosität und die räumliche Abtrennung voneinander bewirkten, dass nationalistische Vorstellungen kaum Bedeutung erlangen konnten.
„Nationalist organizations developed, and the sheikhs continued to play a role in these and in political leadership. The decay of the imperial powers allowed a greater degree of great Power penetration, and certain Kurds became involved in the search for a new identity that would reconcile their situation with the possibilities brought by the ongoing momentous political and social changes throughout the region." (O'Shea 2003: 92).

Es steht fest, dass die kurdische Selbstverwaltung in den letzten Jahren eine beachtliche Eigendynamik entwickelt hat, die nicht mehr aufzuhalten ist. Eine absolute Präferenz und Akzeptanz von Parteien als alleinige Interessensvertreter setzte sich durch, wobei geschickter Weise altbekannte Strukturen darin enthalten blieben und doch das nötige demokratische Vokabular im Umgang mit internationalen Akteuren angeboten werden kann.

Folgt man Stansfield, haben die Kurden überhaupt erst durch die zunehmende Selbstverwaltung ein nationales Bewusstsein entwickelt. Auch dies bestätigt, dass sich die (irakisch-) kurdische Identität nicht mehr an einem Groß-Kurdistan zu orientieren braucht. 
Darüber hinaus führt der Kontakt zu den zahlreichen Kurden in der Diaspora dazu, dass das wesentliche Kriterium der nationalen Identität, die räumliche Gebundenheit, verwischt wird und postnationales Gedankengut sich ausbreitet.

„Whilst the Kurds represented immigrants/refugees and a stateless and subordinated people, the states in question were part of the interstate system which had not simply faded away even if its traditional rationale in terms of bounded ideas about and authority over states and nations had been challenged by supra-state and sub-state actors and institutions." (Emanuelsson 2005: 218).

Die gemeinhin verbreitete Ansicht, nationale Bewegungen würden durch Modernisierungsschübe beflügelt und entstünden bei hohem Grad an Modernisierung von selbst, kann bei den irakischen Kurden nicht belegt werden. „Denn die Akzeptanz breiter Bevölkerungsschichten für eine kurdische nationale Weltsicht (...) geht kaum auf einen neuen Modernisierungsschub zurück. Vielmehr hat man es hier mit einer fortschreitenden Zerstörung jeglicher Lebensperspektive durch staatliche Willkür zu tun. " (Behrendt 1993: 400). Die Tatsache, dass sich durchgehend traditionelle Stammes- und Scheichdynastien auf der politischen Bühne prominent beweg(t)en, beweisen noch die derzeit aktiven Eliten. Die Verbindung zwischen Klanen und religiösen Bruderschaften bleibt politisch bestimmend. Entweder der Klan gehört insgesamt einer Bruderschaft an oder deren Mitglieder haben sich ihm angeschlossen. Scheichs und Aghas sind zunehmend deckungsgleich (Franz 1986: 175). Bei genauer Betrachtung zeigt sich ein Gemisch aus verschiedenen Herrschaftslegitimationen der derzeit politisch Aktiven, hinter denen jeweils gesellschaftlich tragende Gruppen stehen. Talabani, der sich auf die Sufi-Tradition der lokalen Qadiriya aus der Region um Kirkuk stützt, war lange Zeit Kontrahent Barzanis, der sich vor allem auf Stammeszugehörigkeit aus der Barzan-Region des nördlichen Iraks beruft. Das alte Bündnis der Barzanis mit BarzinjiScheichs, den alte Qadiriya-Kontrahenten der Talabanis, mag noch immer durchschimmern.

Trotz alledem ist die wirtschaftliche Umgestaltung, der Verlust feudaler Strukturen prägend für das politische Gefüge, denn verschiedene Ideologien wurden sich verschiedentlich zu eigen gemacht, um das nicht zu fassende Gebilde Kurdistan zu gestalten. Phasenweise gab es Partei-Bündnisse mit arabischer Opposition des Iraks, die nach Veränderung der Regierung zu kurzfristigen Annäherungen an die Zentralgewalt führen konnten.

Mit dem Verweis darauf, dass die Komplexität der gesellschaftlichen Realität der Kurden sich nicht mit der üblichen Terminologie einfach wiedergeben lässt, sollte klar sein, dass im Umkehrschluss ebenso die nationalstaatliche Terminologie für Kurden befremdlich gewesen sein und mit Leben gefüllt werden muss. Die Vermischung von bekannter tribaler, 
wenngleich selbst durchmischter, Elitenvorstellung und moderner staatlicher Organisation befindet sich in einem unsicheren Stadium, da sich offenbar keines von beiden durchzusetzen vermag. Es bleibt abzuwarten, ob tatsächlich die zunehmende Nationalisierung IrakischKurdistans auch ideologisch nationalstaatlichen Charakter annehmen kann, wenn doch jetzt bereits das Interesse am Staat als wichtigster Identifikationsgröße abzunehmen scheint.

„In addition to the questions of ethnic, religious and linguistic division, Kurdish society is driven by other divisions, which have all been factors in the failure of Kurdistan to achieve statehood, and to create a unified imagined community. The Kurdish nationalist movement is seriously challenged by its denial of the diversity of interests and allegiances within the present nationalist movement, and also the historic tensions within Kurdish society and Kurdistan's territory."(O'Shea 2003: 40)

\section{Ausblick}

Wie bereits in der Einleitung festgestellt wurde, sollte diese Arbeit verstanden werden als Grundlage für sich möglicherweise anschließende Feldforschungen. Die dargestellten Aspekte kurdischer Gesellschaft und Beeinflussung ihrer Zusammensetzung durch nationalstaatliche Prozesse bieten gewiss genügend Anregungen für zu bearbeitende Fragestellungen, denen bislang die Daten fehlen. Mithilfe eines längeren Aufenthaltes können beispielsweise auch Daten über das Vorhandensein und die Nutzung moderner Medien und Kommunikationsmittel erhoben werden.

Interessant wäre es einen Überblick über die derzeit vorzufindenden familiären und tribalen Strukturen zu schaffen. Wichtig wäre zu fragen, wie viel und welche Strukturen nach all den Krisen „überlebt“ haben in der fragmentierten Gesellschaft. Zu skizzieren wären hier vor allem Verwandtschaftssysteme und an Positionen gebundene Aufgaben, so wie YalçinHeckmann 1991 dies beispielhaft bei den Hakkari in der Südtürkei vollzogen hat.

Einen wichtigen Einblick in indigenes politisches Denken und Herrschaftslegitimationen könnte die Untersuchung der politischen Rhetorik und Programmatik der Parteien liefern. Die nähere Beleuchtung der charismatischen Führungsfiguren, ihrer Biografien und ihrer vielfältigen gesellschaftlichen Rollen könnte sicherlich einen wichtigen Aufschluss geben über Ideale der Gruppenorganisation. Dies alles könnte einen in die Lage versetzen, die tatsächliche Demokratisierung des Bundesstaats Irakisch-Kurdistan zu bewerten.

Was hier nur angerissen werden konnte, ist außerdem die Rolle der Peschmerga. Da jene durch die letztjährigen Ereignisse noch an Prestige gewonnen haben dürften, sollte der Frage 
nachgegangen werden, ob und wenn ja, welche Zusammenhänge es zwischen der tribalen Ordnung und der Rekrutierung der Kampfverbände noch gibt.

Nicht zuletzt mag die Rolle des Islam in der kurdischen Gesellschaft ein aktuelles und spannendes Thema sein, denn er scheint an Gewicht in der Selbstdefinition zu verlieren, insofern neue extremistische Feindbilder sich über den islamischen Charakter ausweisen und weitere religiöse Identifikationsmöglichkeiten zur Verfügung stehen. 


\section{Anhang}

$\underline{\text { I.a Die Wilayats des Osmanischen Reichs - späterer Irak }}$
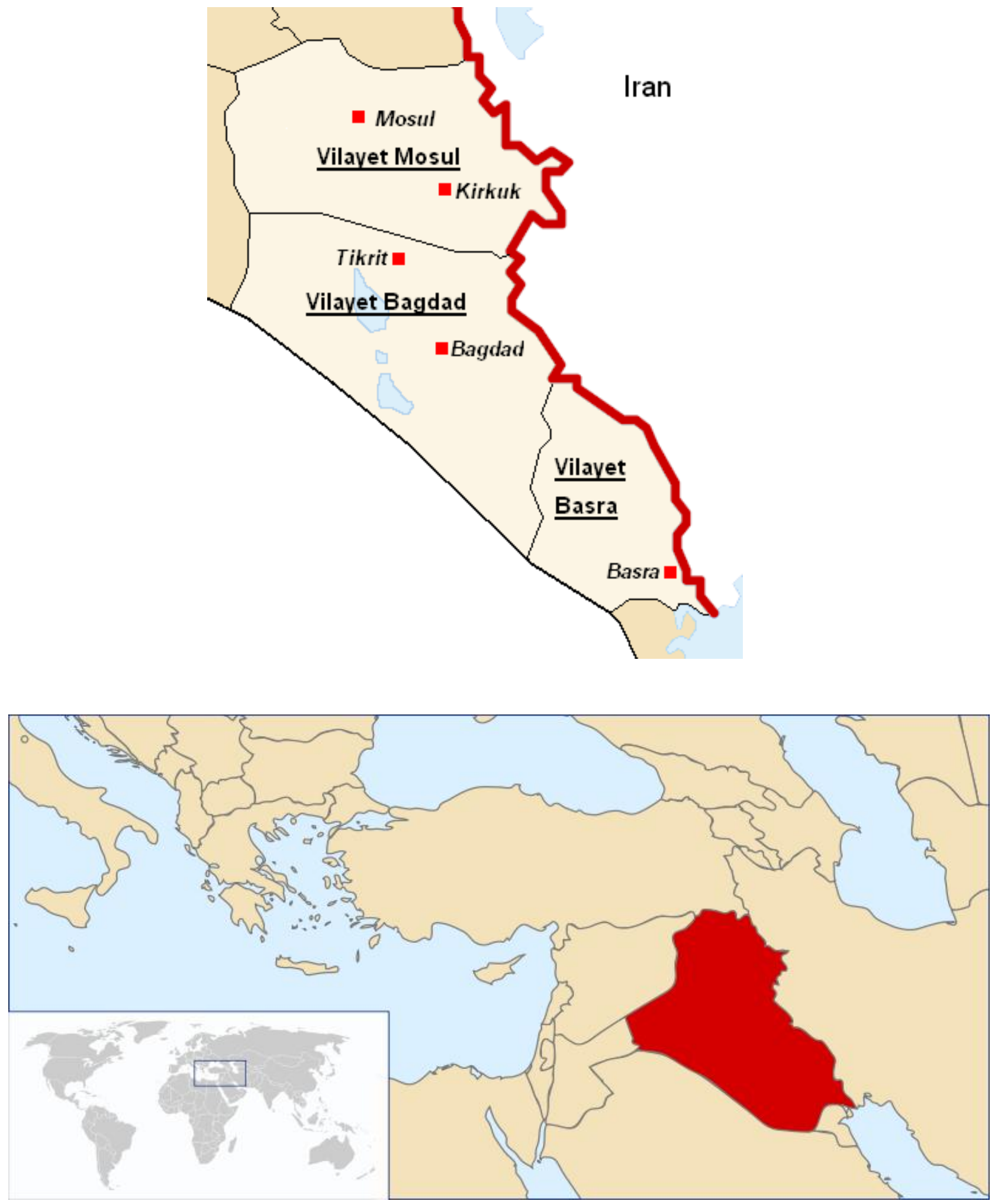

Quelle: Eigene Bearbeitung des Wikipedia-Map-Bearbeitungsprogrammes unter Berücksichtigung des Historischen Weltaltlas 2007: 108-109 


\section{I.b Die Region Irakisch-Kurdistan}

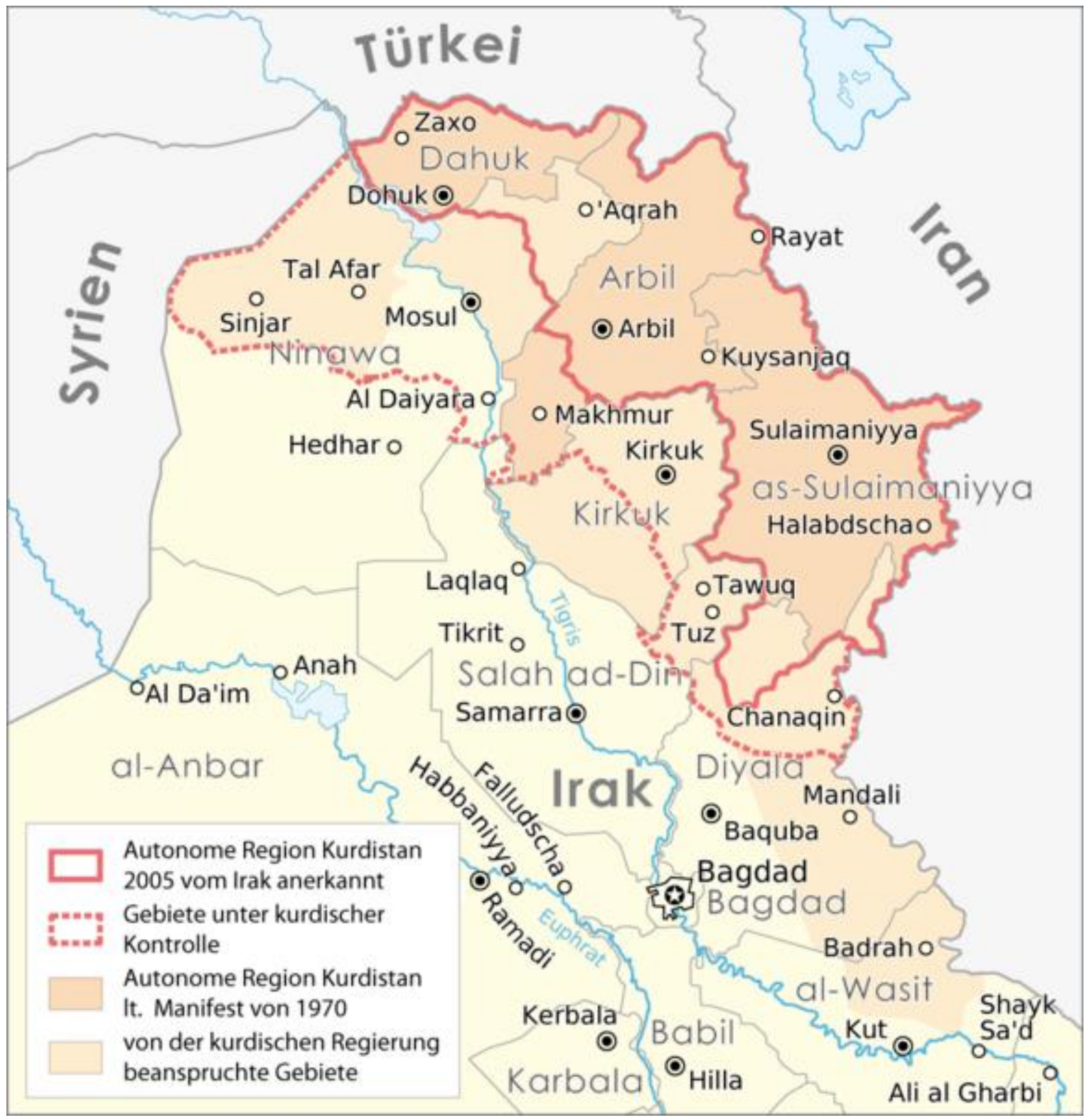

Quelle: Dörrbecker, Maximilian 2007, veröffentlicht bei wikipedia.de 


\section{$\underline{\text { II.a Kurdische „Gruppen“ im Irak }}$}

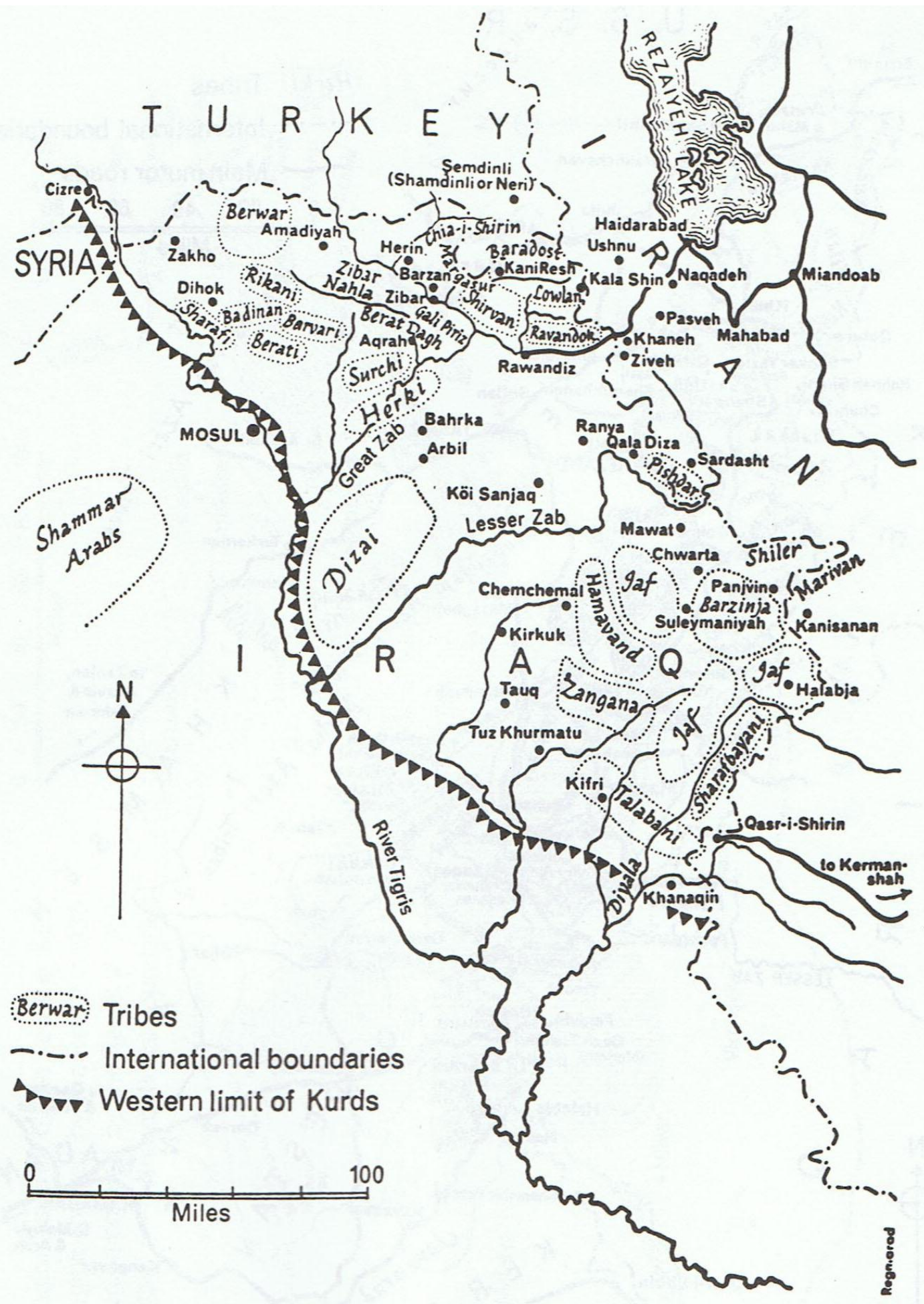

Abb. 3: The Kurds in Iraq

(c) Hugh Edmonds)

Quelle: Günther/Brentjes 2001, S. 158 


\section{II.b Kurdische Stämme}

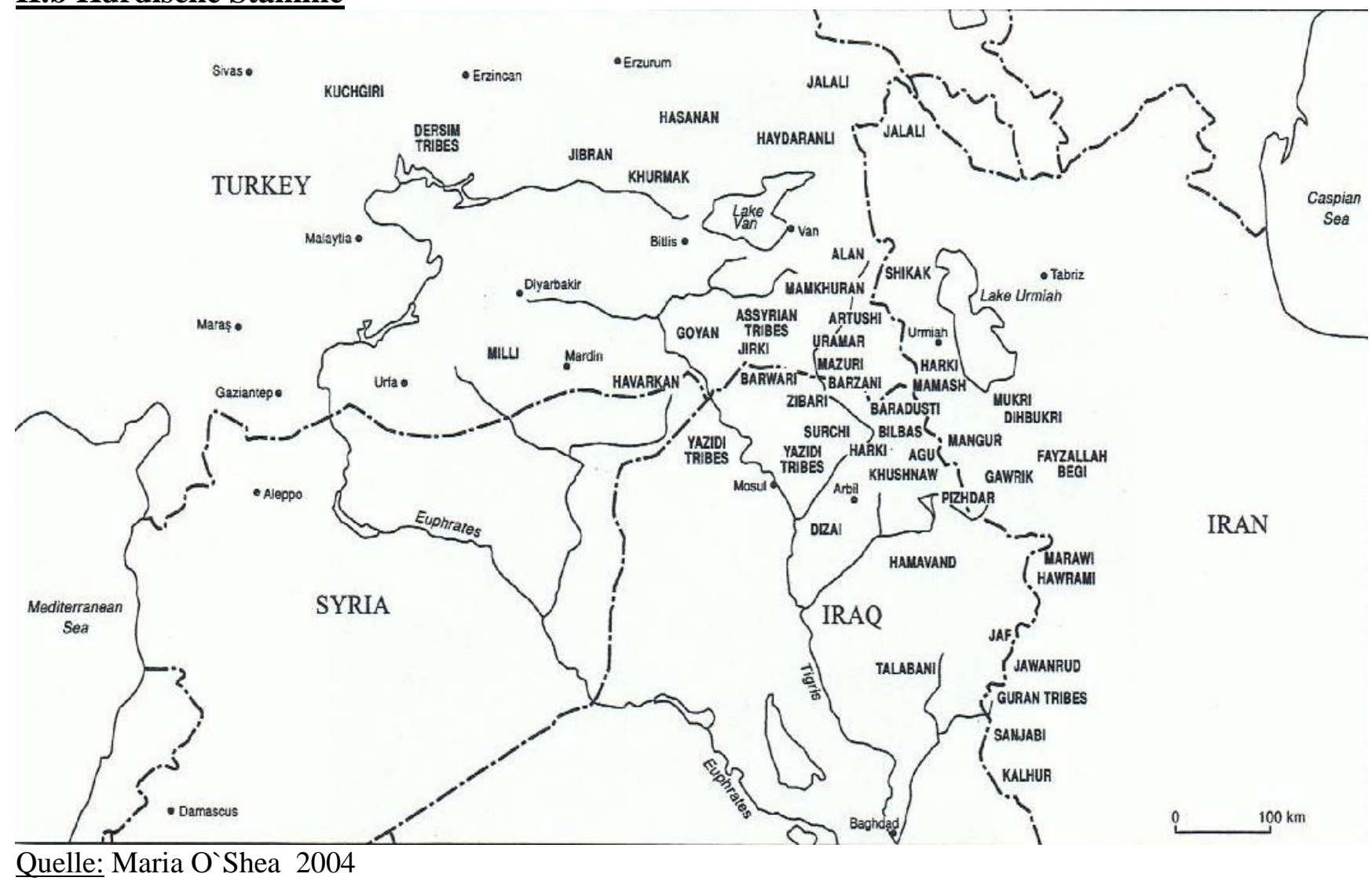

\section{III.a Kurdische Sprachen}

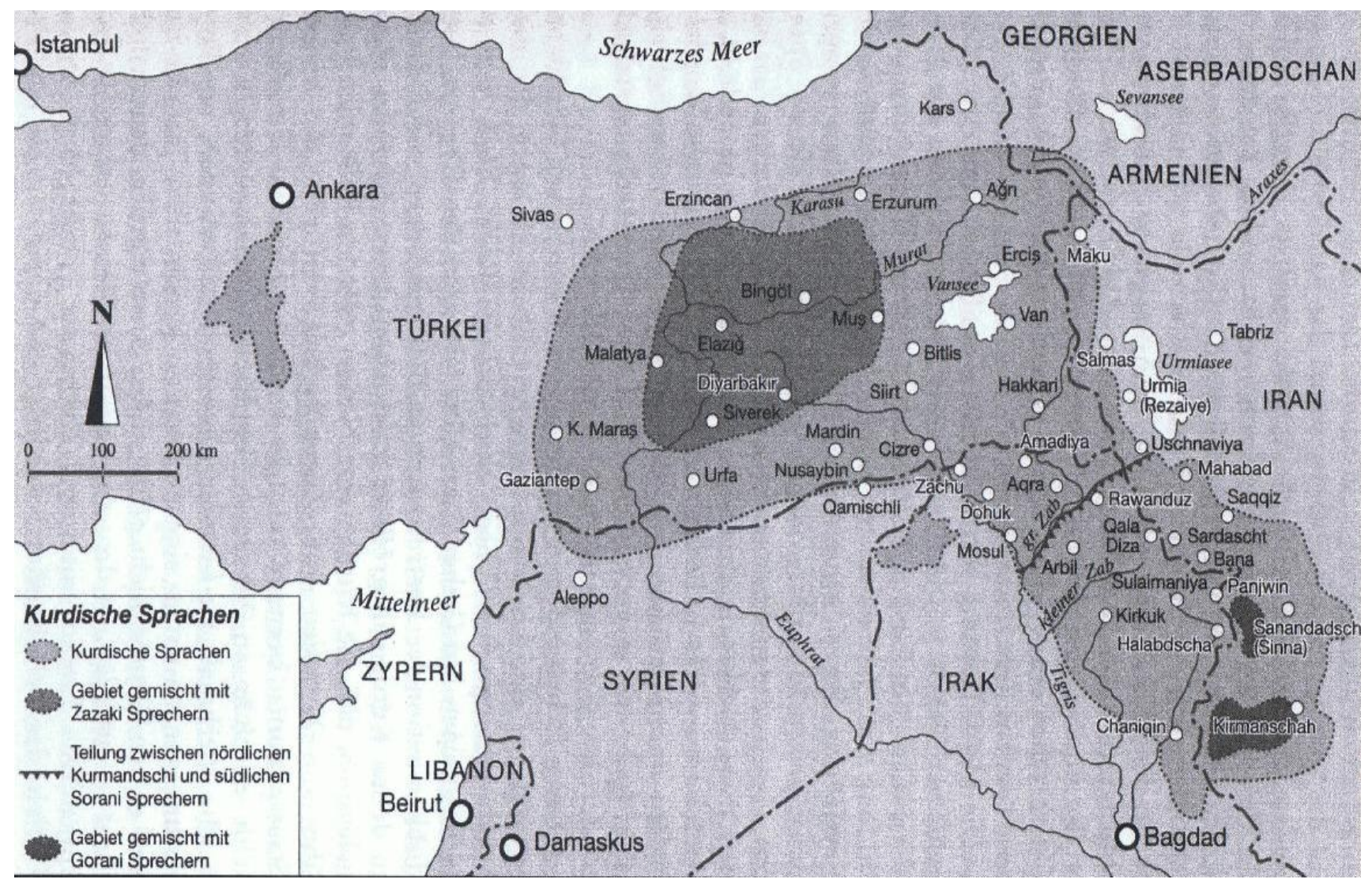

Quelle: Strohmeier 2003 


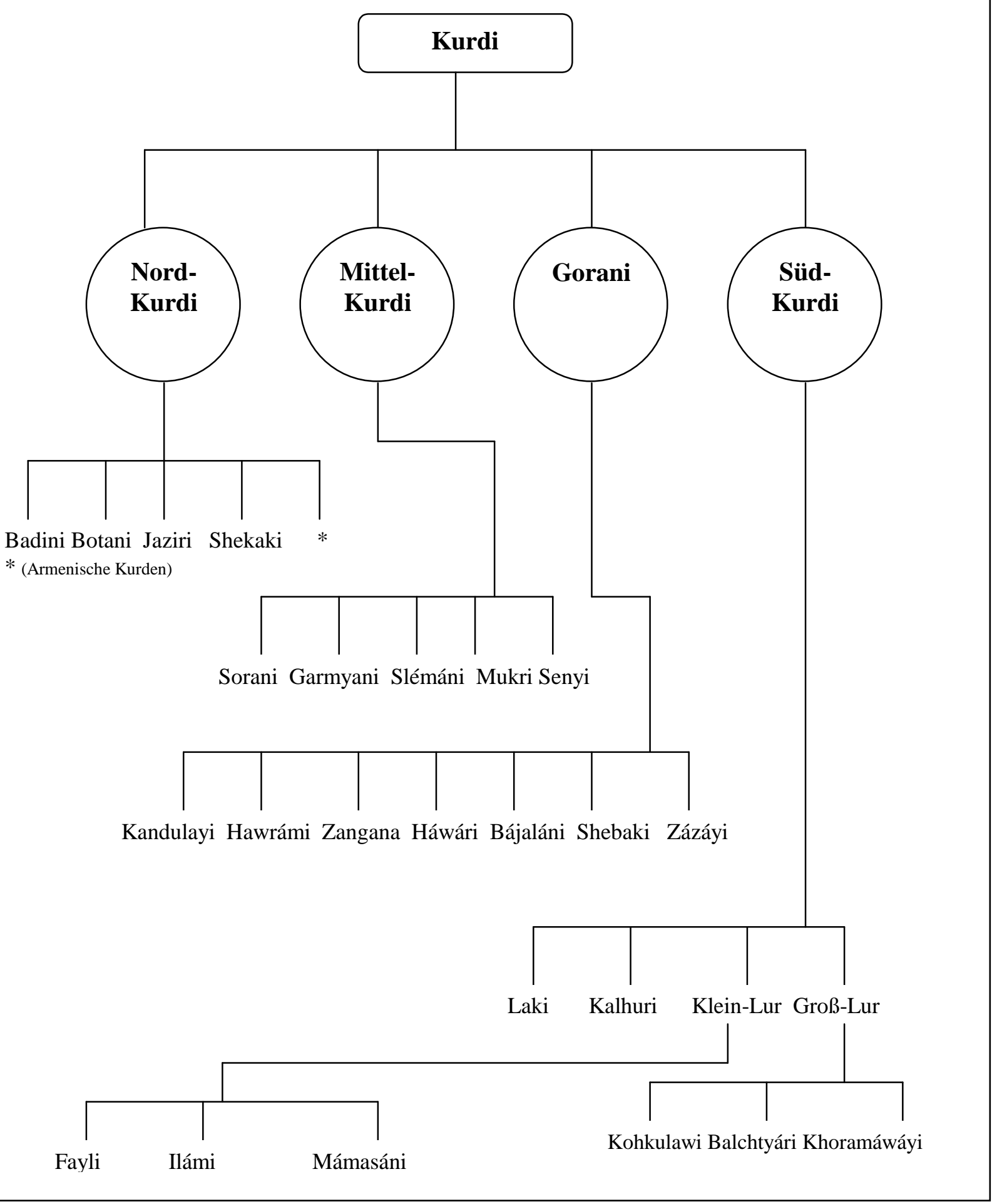

Angemerkt sei, dass unter europäischen Linguisten vermehrt dem Vorhandensein von nord-, zentral- und südkurdischen Dialekten zugestimmt wird. Luri und Gorani werden allerdings von ihnen auch als eigenständige Sprachen bezeichnet. 


\section{$\underline{\text { IV.a Irakische Bevölkerung nach Religionszugehörigkeiten }}$}

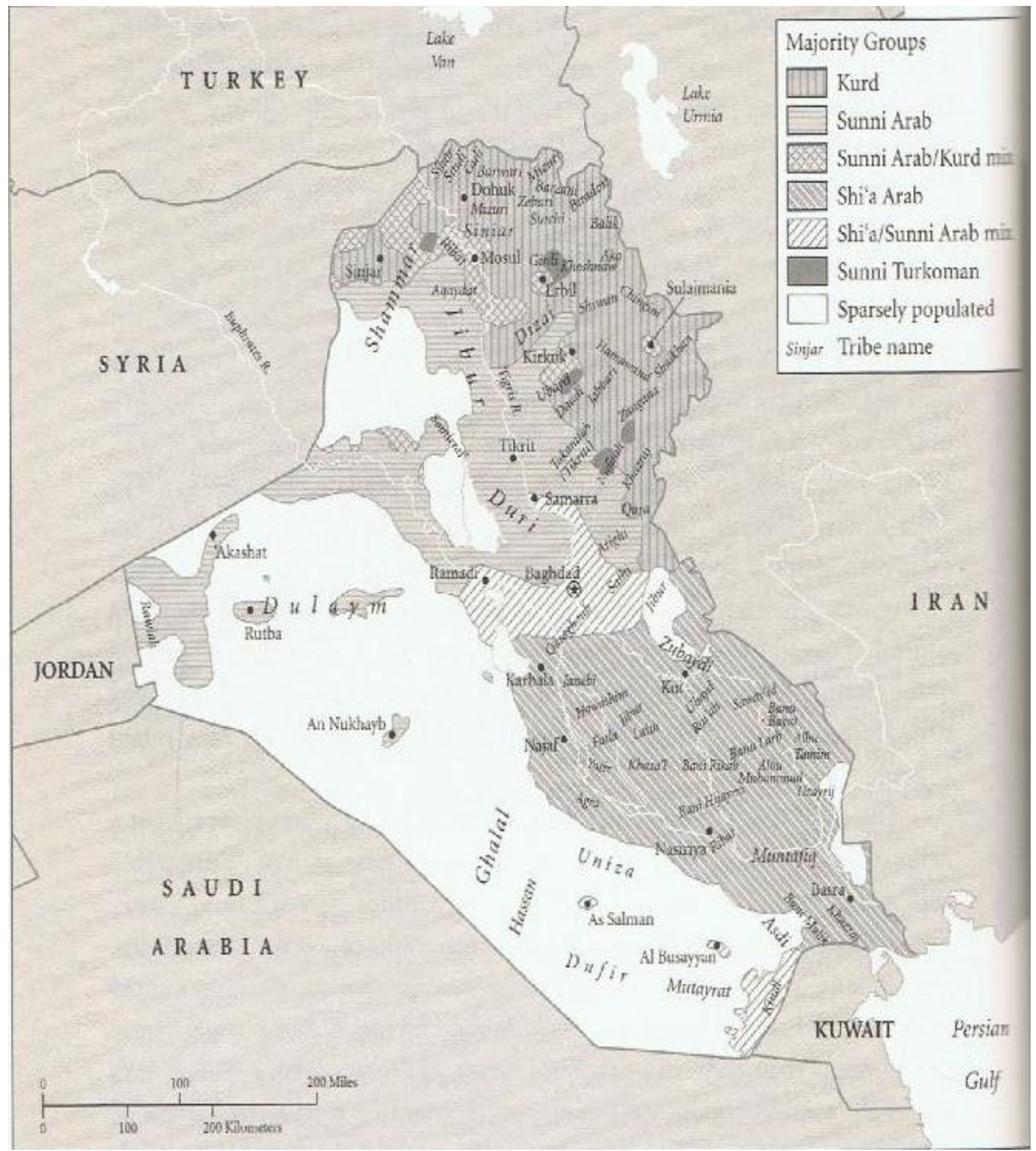

Quelle: Brendan O'Leary 2005 


\section{IV.b Religionen der Kurden}

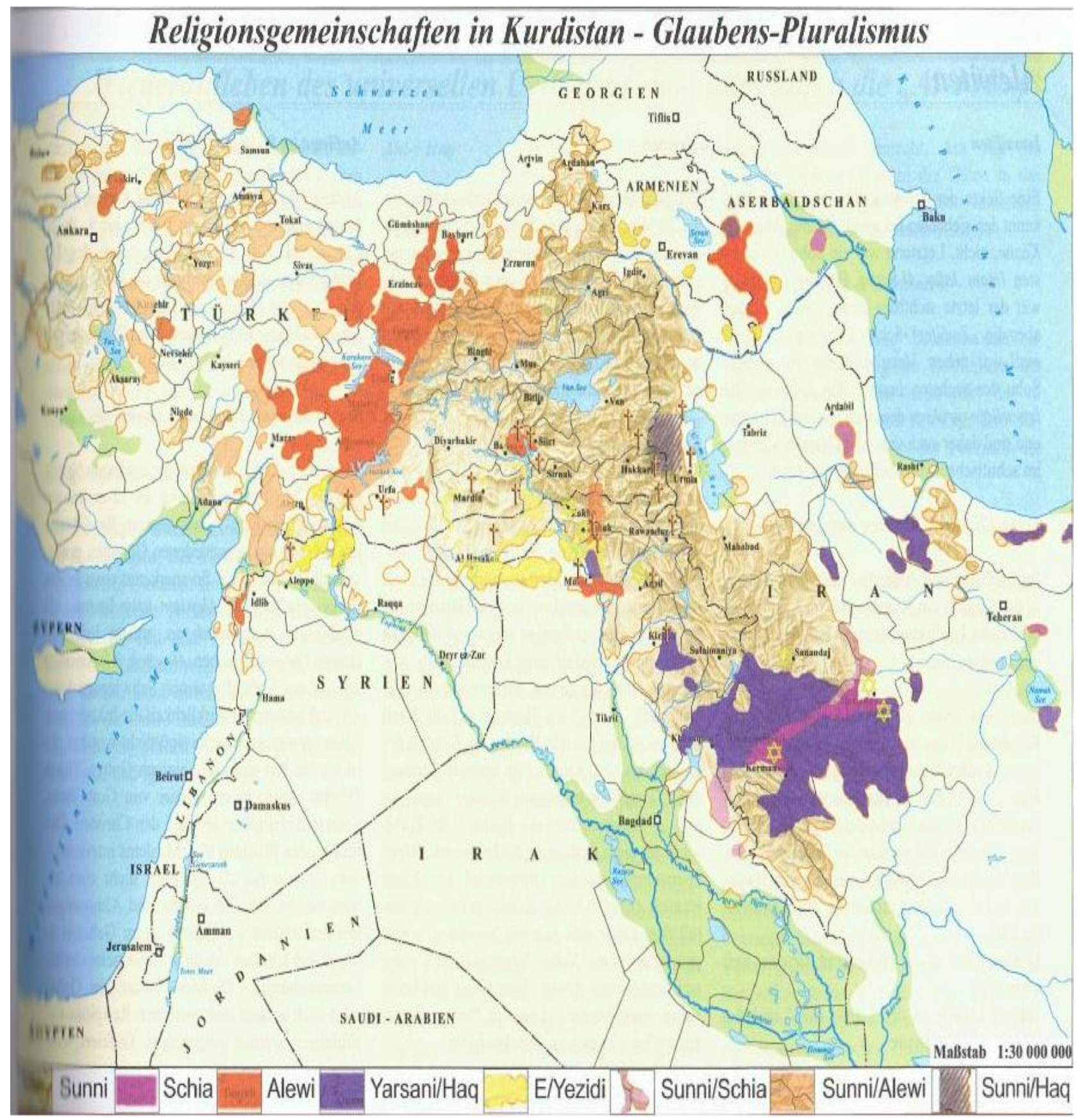

Quelle: Hennerbichler 2004 


\section{Kurdistan der Parteien 1991}

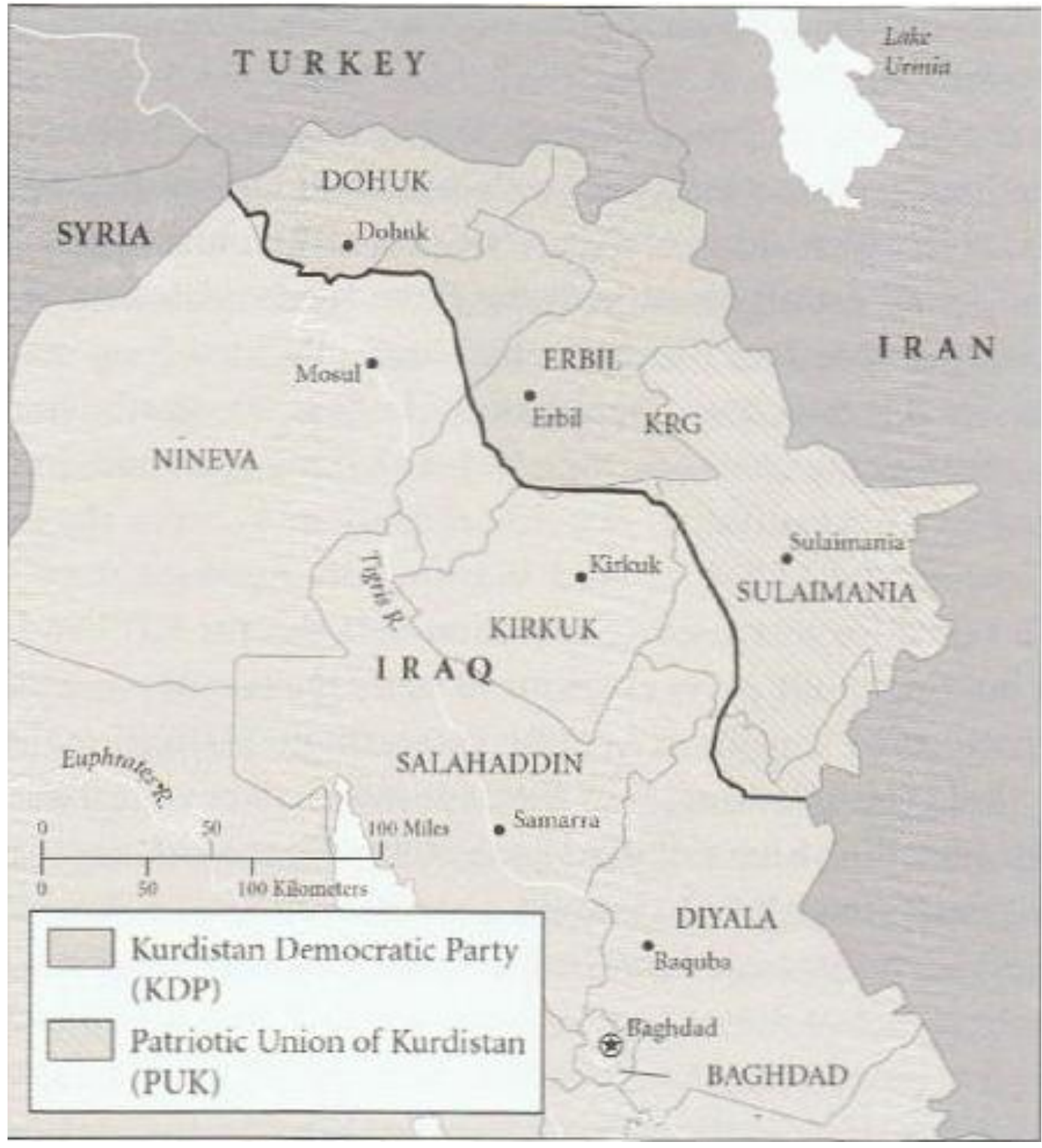

Quelle: Brendan O'Leary 2005 


\section{Erdölregion Kurdistan}

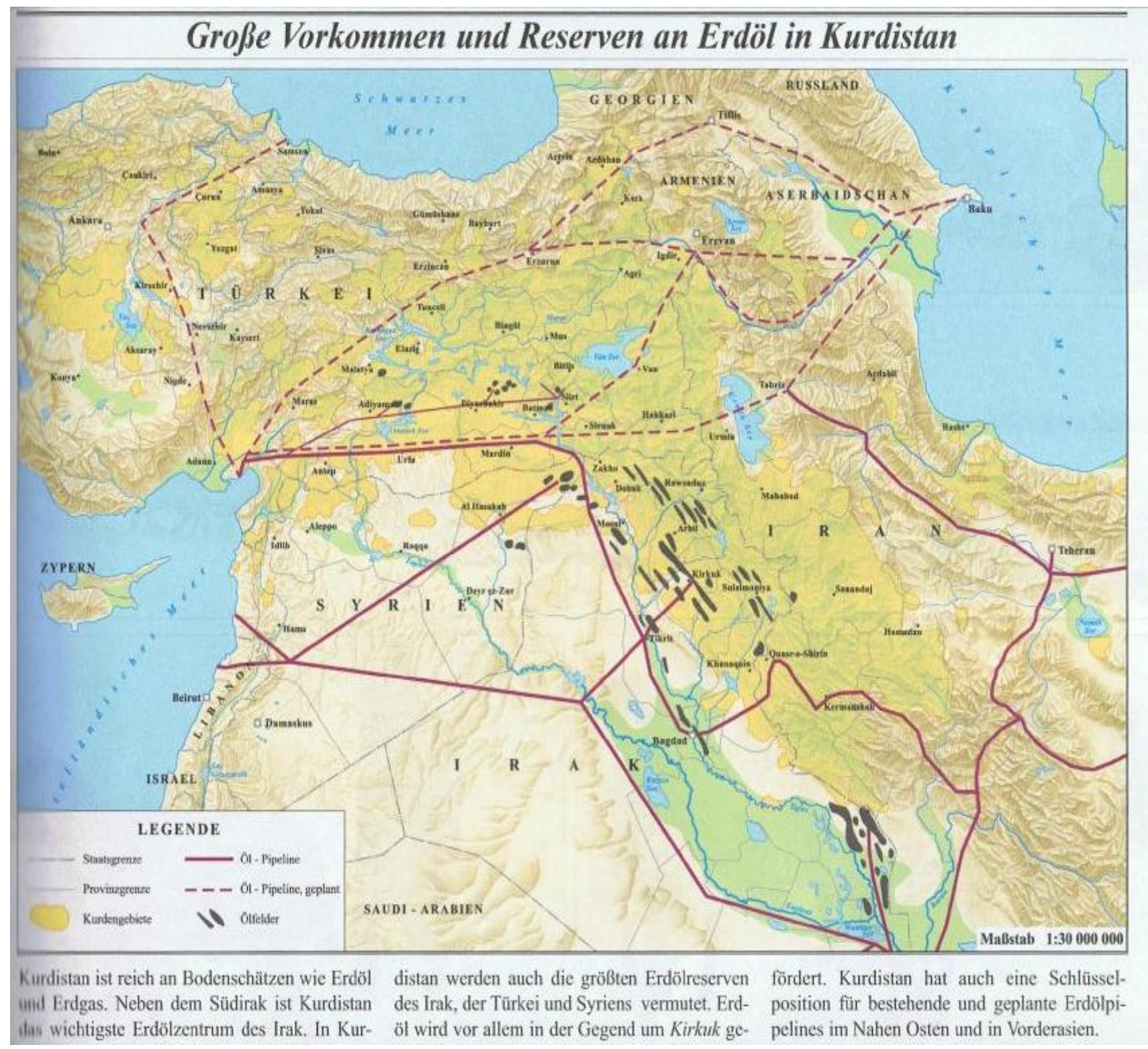

Quelle: Hennerbichler 2004 


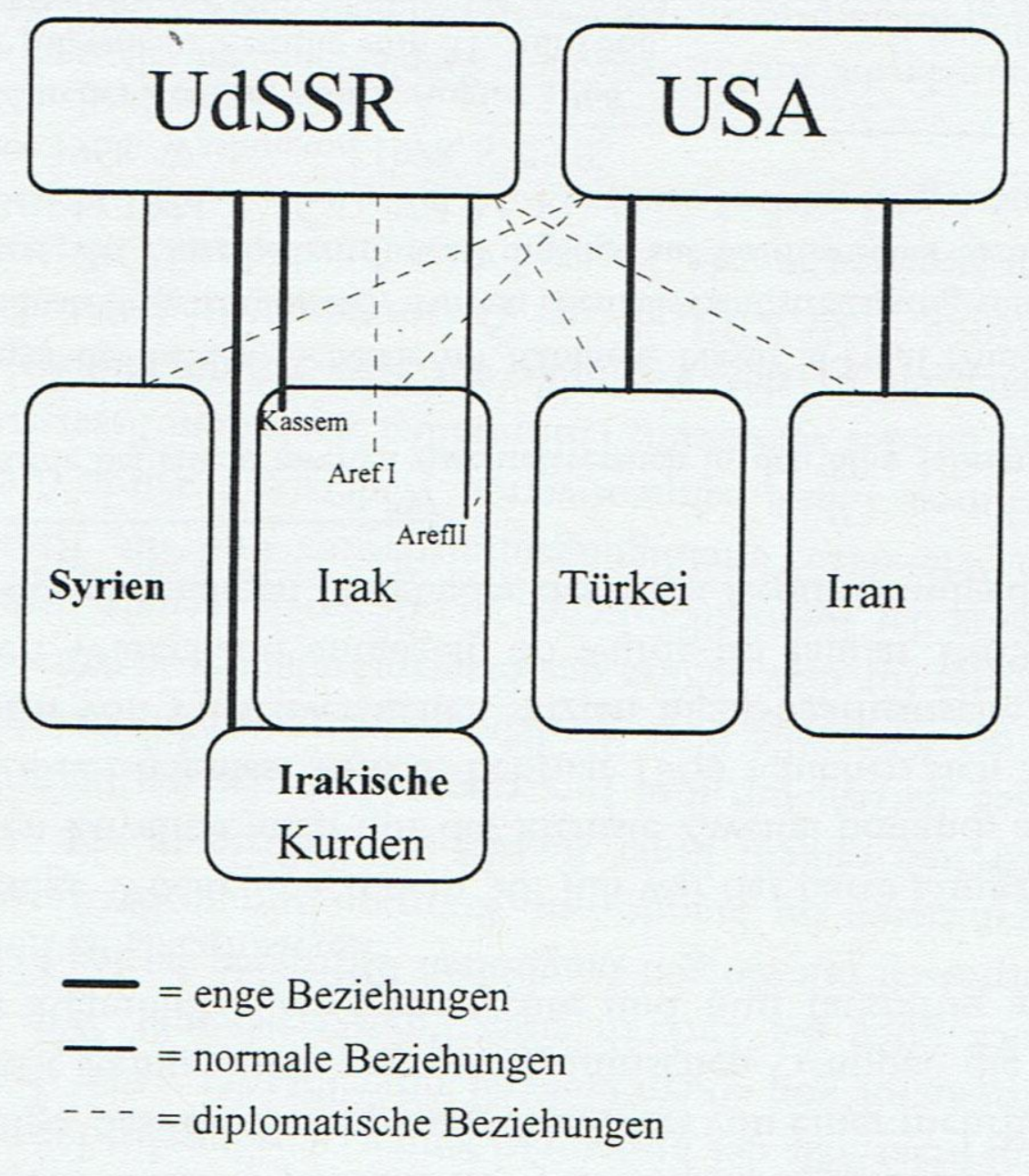

Quelle: Asadi 2007:275 


\section{Parteien um 1980 im Überblick}

Nach dem Algerien-Abkommen

Abb. 2:

Überblick über die Parteienlandschaft in Irakisch-Kurdistan zwischen 1975-1988

Die Organisationen sind in der Reihenfolge ihrer Gründungsdaten aufgeführt

- Patriotische Union von Kurdistan (PUK) (01.06.1975) anfänglich aus 3 später aus 2 Unterorganisationen

\begin{tabular}{|c|c|c|}
\hline $\begin{array}{c}\text { Marxistisch-Leninistischer } \\
\text { Bund Kurdistans } \\
\text { (10. Juni 1970); } \\
1978 \text { umbenannt in: } \\
\text { Bund der Werktätigen Kurdistans } \\
\text { (BWK) }\end{array}$ & $\begin{array}{c}\text { Progressive } \\
\text { Patriotisch- } \\
\text { Revolutionäre } \\
\text { Breite Tendenz } \\
1977 \\
\text { (Allgemeine Linie) }\end{array}$ & $\begin{array}{c}\text { Sozialistische } \\
\text { Bewegung Kurdistans } \\
\text { (SBK) } 1976 / 77\end{array}$ \\
\hline $\begin{array}{l}\text { BWK } \\
\text { vom BWK gespalten: a) Organisation } \\
\text { der Werktätigen Kurdistans 1975/76 } \\
\text { gespalten (eine großangelegte Verhaf- } \\
\text { tungswelle erfasste die meisten Mitglie- } \\
\text { der und führte deren Ende herbei) } \\
\text { b) Flagge der Revolution (1985 gespal- } \\
\text { ten. Ende der 80er, Anfang der 90er } \\
\text { Jahre schloss sich die PUK nochmals } \\
\text { zusammen) }\end{array}$ & \multicolumn{2}{|c|}{$\begin{array}{l}\text { Union der Revolutionären von Kurdistan } \\
\text { Ein am 15.08.1983 erfolgter Zusammen- } \\
\text { schluss } \\
\text { zwischen der Allgemeinen Linie und der SBK }\end{array}$} \\
\hline
\end{tabular}

- Kurdische Nationalistische Sozialistische Partei (01.09.1975) umbenannt am 01.09.1976 in Nationalistische Partei der Kurden (NPK)

- Demokratische Partei Kurdistans - Provisorische Führung (DPK-PF) (10.12.1975). 1979 erneut umbenannt in Demokratische Partei Kurdistans Irak (DPK)

- Demokratische Partei Kurdistans - das Vorbereitende Komitee (DPKVK) 1976/77 gründete zusammen mit der am 21.03.1979 mehrheitlich von der PUK gespaltenen Sozialistischen Bewegung Kurdistans (SBK) die Vereinigte Sozialistische Partei Kurdistans (08.08.1979). 08.08. 1980 umbenannt in Sozialistische Partei Kurdistan-Irak (SPK-I)

- Demokratische Union Kurdistan (DUK) (09.06.1977)

- Islamische Armee der Kurden (1980)

- Islamische Bewegung Kurdistan-Irak (1981?)

- Demokratische Volkspartei Kurdistan (1981). Eine von der DPK gespaltene Gruppierung

- Demokratisch-Revolutionäre Richtung der Sozialistischen Partei Kurdistans-Irak gespalten von SPK-I im Sommer 1985. 1988 umbenannt in Partei der Werktätigen von Kurdistan-Irak 


\section{$\underline{\text { IX. Positionen - Prestige }}$}

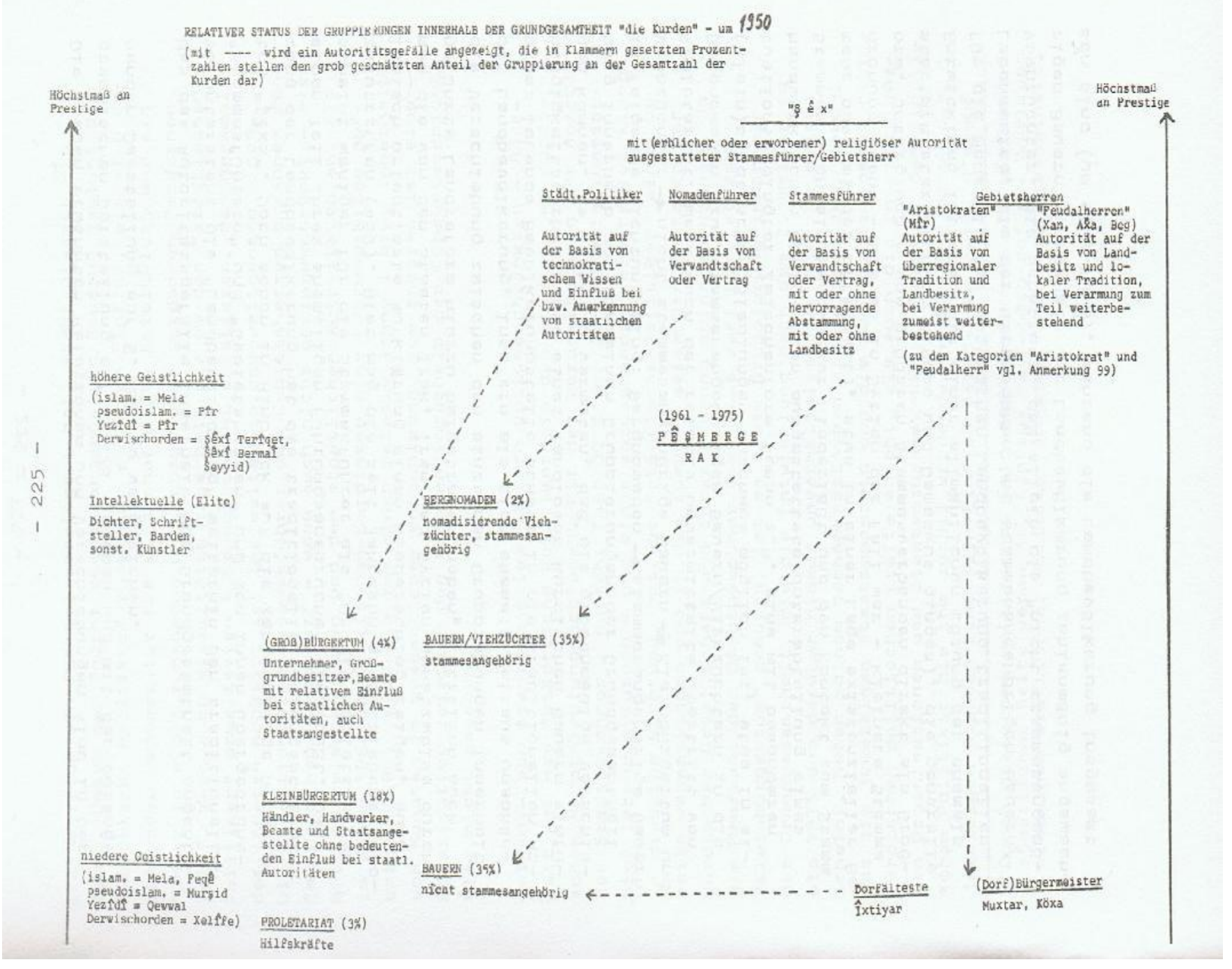

Quelle: Küchler 1978 


\section{Literaturverzeichnis}

Ağuiçenoğlu, Hüseyin

1997 Genese der türkischen und kurdischen Nationalismen im Vergleich. Vom islamisch-osmanischen Universalismus zum nationalen Konflikt. Münster: Lit Verlag. (Heidelberger Studien zur Internationalen Politik, 5)

Appadurai, Arjun

1996 Modernity at Large: Cultural Dimensions of Globalization. Minnesota: Univ. of Minnesota

Press.

Asadi, Awat

2007 Der Kurdistan-Irak-Konflikt. Berlin: Hans Schiller Verlag.

Ascherson, Neal

2004 Politik und Ethnizität im 21. Jahrhundert. Europäische Rundschau .3: 85-95.

\section{Bahjat, Sarbest}

2001 Die politische Entwicklung der Kurden im Irak von 1975 bis 1993 unter besonderer Berücksichtigung von Saddam Husseins Kurdenpolitik. Berlin: Klaus Schwarz Verlag. (Islamkundliche Untersuchungen, 237)

\section{Barth, Erik}

1979 Principles of Social Organization in Southern Kurdistan. Neuaufl. New York: AMS Press

1998 Ethnic Groups and Boundaries. The social Organization of Cultural Difference. Neuaufl.; S. 938. Long Grove: Waveland Press.

\section{Behrendt, Günter}

1993 Nationalismus in Kurdistan. Vorgeschichte, Entstehungsbedingungen und erste Manifestationen bis 1925. Hamburg: Deutsches Orient-Institut.

\section{Bell, Wendell}

1974 Ethnicity and Nation-Building: Comparative, International, and Historical Perspectives. Beverly Hills: Sage.

\section{Bengio, Ofra}

2005 Autonomy in Kurdistan in Historical Perspective. In: Brendan O'Leary (Hg.), The Future of Kurdistan in Iraq. S. 173-186. Philadelphia: University of Pennsylvania Press.

\section{Braidwood, Robert}

1998 From Cave to Village in Iraq. Agricultural History 28:2, S.41.

\section{Brentjes, Burchard}

2001 Die Kurden. Ein Abriss zur Geschichte und Erfahrungsberichte zur aktuellen humanitären Situation. Wien: Braumüller.

\section{Craf, John}

1996 Iraq, Land of the Nomad. Social Studies 35:7, S. 305-308.

\section{Dawn, Ernest}

1991 The Origins of Arab Nationalism. In: Rashid Khalidi (Hg.), The Origins of Arab Nationalism. New York: Columbia University Press.

\section{Deschner, Günther}

2003 Dir Kurden. Volk ohne Staat. München: Herbig. 
Dodge, Tony

2003 Inventing Iraq. London: Hurst \& Company.

Donnan, Hastings und Wilson, Thomas

1999 Borders - frontiers of identity, Nation and State. Oxford: Berg.

Dorman, James

1980 Ethnic Groups and "Ethnicity": Some Theoretical Considerations. Journal of Ethnic Studies 7:4, S. 23-36.

\section{Edmonds, C.J.}

1957 The Kurds of Iraq. Middle East Journal 11:1, S. 52.

1971 Kurdish Nationalism. Journal of contemporary History 6:1, S. 87-107.

\section{Eichhorst, Kristina und Sinjen, Svenja}

2006 Die Irakische Verfassung ein geeignetes Mittel zur Lösung der ethnischen Konflikte? Politische Studien 57: 405f.

\section{Elwert, Georg}

2002 Switching identity discourse: primordial emotions and the social constructions of we-groups. In: Imagined differences. S. 33-56. Münster: Lit-Verlag.

1989 Ethnizität und Nationalismus: über die Bildung von Wir-Gruppen. Kölner Zeitschrift für Soziologie 3: 440-464.

\section{Emanuelsson, Ann-Catrin}

2005 Diaspora Global Politics. Kurdish transnational networks and accomodation of nationalism. Göteborg: Intellecta Docusys.

\section{Eriksen, Hylland T.}

2002 Ethnicity and Nationalism. London: Pluto Press.

\section{Estel, Bernd}

1997 Moderne Nationsverständnisse: Nation als Gemeinschaft. In: Robert Hettlage und Petra Deger (Hg.), Kollektive Identität in Krisen. Ethnizität in Religion, Nation, Europa; S. 73-86. Opladen: Westdeutscher Verlag.

\section{Faist, Thomas}

2000 Jenseits von Nation und Post-Nation. Zeitschrift für Internationale Beziehungen 7: 109-144.

\section{Fischer-Tahrir, Andrea}

2003 Frauen in Irakisch-Kurdistan. In: NAVEND - Zentrum für Kurdische Studien e.V. (Hg.), Kurden heute. Bonn: NAVEND.

\section{Francis, E.K.}

1968 The Ethnic Factor in Nation-Building. Social Forces, 46:3, S. 338-346.

Franz, Erhard

1986 Kurden und Kurdentum. Zeitgeschichte eines Volkes und seiner Nationalbewegungen. Hamburg: Deutsches Orient-Institut.

\section{Friedmann, John}

1967 Regional Planning and Nation-Building: An Agenda for International Research. Economic and Cultural Change 16: 119-129.

Fuller, Graham

1993 The Fate of the Kurds. Foreign Affairs 72: 108-121. 
Geis, Anna

2001 Literaturbericht. Diagnose Doppelbefund - Ursache ungeklärt? Die Kontroversen um den Demokratischen Frieden. Politische Vierteljahreszeitschrift 41: 283-297.

\title{
Giordano, Christian
}

1997 Ethnizität: Prozesse und Diskurse im interkulturellen Vergleich. In: Robert Hettlage und Petra Deger (Hg.), Kollektive Identität in Krisen. Ethnizität in Religion, Nation, Europa; S. 56-73. Opladen: Westdeutscher Verlag.

\section{Goodenough, Ward H.}

1996 Rethinking Status and role. Toward a General Model of the Cultural Organization of Social Relationships. London: Tavistock Publications.

\section{Griffith, Anthony}

1990 Social Studies for Nation Building. Social Studies 81:4, S. 160.

\section{Haller, Dieter}

2005 dtv-Atlas Ethnologie. München: dtv.

\section{Hauser-Schäublin, Brigitta und Braukämper, Ulrich}

2002 Ethnologie der Globalisierung. Perspektiven globaler Verflechtungen. Berlin: Reimer.

\section{Havrest, Latif}

1998 Sprachpolitik, Sprachenrecht und Sprachplanung im geteilten Kurdenland. Wien: Passagen Verlag.

\author{
Hechter, Michael \\ 2000 Containing Nationalism. Oxford: Oxford University Press.
}

\section{Heckmann, Friedrich}

1997 Ethnos - eine imaginierte oder reale Gruppe? Über Ethnizität als soziale Kategorie. In: Robert Hettlage und Petra Deger (Hg.), Kollektive Identität in Krisen. Ethnizität in Religion, Nation, Europa; S. 46-56. Opladen: Westdeutscher Verlag.

\section{Heidemann, Frank}

2003 Politikethnologie. In: Ethnologie. Einführung und Überblick; S.157-178. Berlin: Reimer.

\section{Hennerbichler, Ferdinand}

2004 Die Kurden. Mosonmagyaróvár: Eigenverlag des Autors.

\section{Hippler, Jochen}

2004 Nation-Building. Ein Schlüsselkonzept für friedliche Konfliktbearbeitung? Ulm: Ebner \& Spiegel.

\section{Heintze, Hans-Joachim}

2003 Die Kurden im Irakkonflikt. Blätter für deutsche und internationale Politik 4: 446-455.

\section{Hobsbawm, Eric}

2005 Nationen und Nationalismus.3.Aufl.; Frankfurt a.M.: Campus Verlag.

\section{Hottinger, Arnold}

1965 Der Kampf der irakischen Kurden um die Autonomie. Europa-Archiv. Zeitschrift für Internationale Politik 12: 465-475.

\section{Jenkins, Richard}

2008 Social Identity. 3.Aufl.; New York: Routledge. 


\section{Küchler, Hannelore}

1978 Eine theoretische Betrachtung und eine exemplarische Untersuchung zum Selbstverständnis der Kurden. Inaugural-Disseratation der Freien Universität Berlin.

\section{Klugman, Marcel}

2002 Europäische Menschenrechtskonvention und antiterroristische Maßnahmen. Frankfurt am Main: Peter Lang Verlag. (Europäische Hochschulschriften 3315)

\section{Kreyenbroek, Philip und Allison, Christine}

1996 Kurdish Culture and Identity. London: Zed Books.

\section{Makiya, Kanan}

1992 The Anfal: Uncovering an Iraqi campaign to exterminate the Kurds. Harper`s 281:1704: 53-60.

\section{Meier, Andreas}

1995 Politische Strömungen im modernen Islam. Wuppertal: Peter Hammer Verlag.

\section{Müller, Ernst}

1996 Gruppe. In: Wörterbuch der Völkerkunde. Walter Hirschberg (Hg.); 2. Auflage. Berlin: Reimer.

\section{Naamani, Israel}

1966 The Kurdish Drive for Self-Determination. The Middle East Journal, 20:3, S. 279-295.

\section{O'Shea, Maria}

2003 Trapped Between the Map and Reality. Geography and Perceptions of Kurdistan. London: Routledge.

\section{Orywal, Erwin}

2005 Ethnizität. In: Wörterbuch der Völkerkunde. Walter Hirschberg (Hg.); 2. Auflage. Berlin: Reimer.

\section{Özdemir, Kadir}

2006 Die Kurden - Ein Volk in drei Nationen. Marburg: Tectum.

\section{Prince, James}

1993 A Kurdish State in Iraq? Current History 92:570, S.17-22.

\section{Reinke, Sarah}

2007 Religiöse Vielfalt in Kurdistan. Pogrom. Zeitschrift des Vereins für bedrohte Völker 3: 36-37.

\section{Reiterer, Albert}

1998 Soziale Identität. Ethnizität und sozialer Wandel: Zur Entwicklung einer anthropologischen Struktur. Frankfurt a.M.: Peter Lange Verlag. (Europäische Hochschulschriften Reihe XXII Soziologie. Vol.321)

\section{Rogg, Inga}

2003 Die Irakischen Kurden auf der Suche nach einer Zukunft im Irak. In: NAVEND - Zentrum für Kurdische Studien e.V. (Hg.), Kurden heute. Bonn: NAVEND.

\section{Salih, Azad}

2005 Freies Kurdistan. Die selbstverwaltete Region Kurdistans. Berlin: Köster.

\section{Schlee, Günther}

2006 Wie Feindbilder entstehen: eine Theorie religiöser und ethnischer Konflikte. München: Beck. 


\section{Schopenhauer, Arthur}

2004 Nation. In: Hans W. Wüst, Lexikon der Sprüche und Widersprüche. S.204. Wien: Tosa.

\section{Sharif, Issam}

1992 Die Irakischen Kurden - Tragödie eines Volkes. Wien: Hans Schiller Verlag.

\section{Smutek-Riemer, Andrea}

1996 Die Kurden. Berlin: Peter Lang Verlag.

\section{Stansfield, Gareth}

2005 Governing Kurdistan: The Strengths of Division. In: Brendan O'Leary (Hg.), The Future of Kurdistan in Iraq. S. 195-218. Philadelphia: University of Pennsylvania Press.

\section{Strohmeier, Martin}

2003 Die Kurden. Geschichte, Politik, Kultur. München: Beck.

\section{Talentino, Andrea}

2005 Collision Course: nation-building, norms, and sovereignty Draft. In: Riegler, Henriette (Hg.) Nation Building between National Sovereignity and International Intervention. Berlin: Nomos.

\section{Turner, Victor}

1974 Dramas. Fields and Metaphors. Symbolic Action in Human Society. Ithaca: Cornell Univ. Press.

\section{van Bruinessen, Martin}

1999 The Kurds in Movement: Migrations, Mobilisations, Communications and the Globalisation of the Kurdish Question. Tokyo: The University of Tokyo. (Islamic Area Studies, No 14)

2000 Mullas, Sufis and Heretics: The role of Religion in Kurdish society. Collected Articles. Istanbul: Isis Press.

\section{Wicker, Hans-Rudolf}

1998 Nationalismus, Multikulturalismus und Ethnizität. Wien: Paul Haupt.

\section{Wimmer, Andreas}

Noch unveröffentlicht. Elementary Strategies for Making Ethnic Boundaries. In: Reader für das Hauptseminar „Dynamik und Konfliktpotenzial ethnischer Grenzziehungen“ im Sommersemester 2007 von Ulrich Braukämper. Göttingen.

\section{Yalçin-Heckmann, Lale}

1991 Tribe and Kinship among the Kurds. Frankfurt a.M.: Peter Lang Verlag. (European University Studies Reihe XIX Ethnologie Vol.27)

\section{Zielke, Barbara}

2007 Sozialer Konstruktivismus. Göttingen: Vandenhoek \& Ruprecht. 


\section{Weitere Quellen:}

Document Statement from the Kurdish Bureau (France)

1962 Middle East Journal, 16:3, S. 373f.

Documents of the American Congress: Armed conflicts

http: // acd.iiss.org/armedconflict/MainPages/dsp_ConflictSummaryy.asp?ConflictID=191

(abgerufen am 20.03.09 in der Bibliothek des Deutschen Bundestages)

\section{Fischer Weltalmanach}

2007 Frankfurt a.M.: Fischer.

\section{Historischer Weltaltlas}

2007 herausgegeben von Dr. Walter Leisering. Wiesbaden: Matrix Verlag.

Homepage der Kurdischen Regionalregierung: www.krg.org

-Liste der Parlamentsabgeordneten:

www.krg.org/uploads/documents/KNAMembers_2006_11_30_h12m44s8.pdf

(abgerufen am 11.5. 09).

Homepage der PUK: www.puk.org

Homepage des Kurdischen Instituts Paris: www.institutkurde.org/kurdorama/ (abgerufen am 18.05.09)

Homepage von Wikipedia.de (für die Gestaltung der ersten angehängten Karten)

\section{International Crisis Group}

2006 Iraq and the Kurds: The Brewig Battle over Kirkuk. (Middle East Report, 56) Internet Content. 2007 Iraq and the Kurds, resolving the Kirkuk Crisis. (Middle East Report, 64) Internet Content. (abgerufen am 21.3. 2009 in der Bibliothek des Deutschen Bundestag) 